\title{
Preirradiation Characterization of MiniFuel Compacts for High-Power Irradiation Testing of TRISO Fuel
}

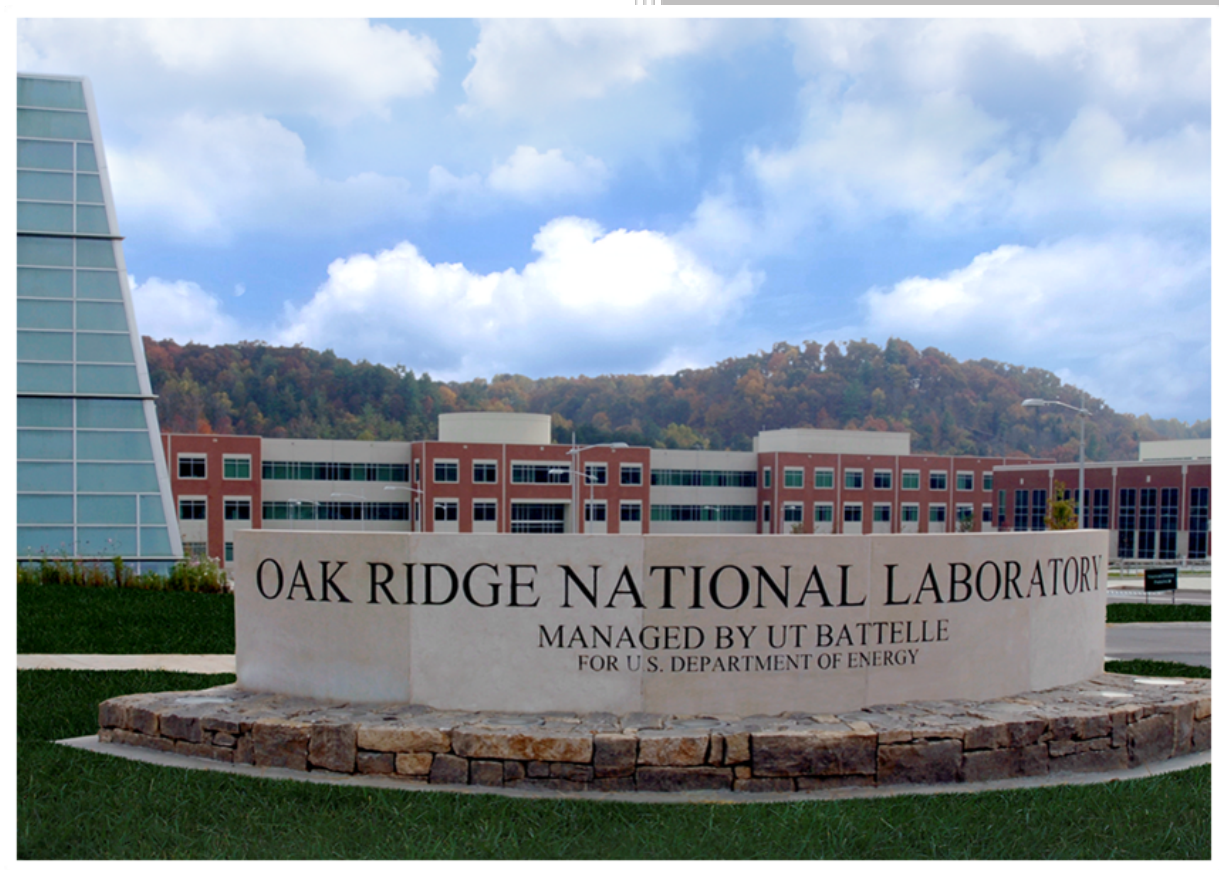

\section{Approved for public release.} Distribution is unlimited

Tyler J. Gerczak Grant W. Helmreich Christopher A. Hobbs Andrew K. Kercher Ryan Latta January 2021 


\title{
DOCUMENT AVAILABILITY
}

Reports produced after January 1, 1996, are generally available free via US Department of Energy (DOE) SciTech Connect.

Website www.osti.gov

Reports produced before January 1, 1996, may be purchased by members of the public from the following source:

\author{
National Technical Information Service \\ 5285 Port Royal Road \\ Springfield, VA 22161 \\ Telephone 703-605-6000 (1-800-553-6847) \\ TDD 703-487-4639 \\ Fax 703-605-6900 \\ E-mail info@ntis.gov \\ Website http://classic.ntis.gov/
}

Reports are available to DOE employees, DOE contractors, Energy Technology Data Exchange representatives, and International Nuclear Information System representatives from the following source:

Office of Scientific and Technical Information

PO Box 62

Oak Ridge, TN 37831

Telephone 865-576-8401

Fax 865-576-5728

E-mail reports@osti.gov

Website http://www.osti.gov/contact.html

This report was prepared as an account of work sponsored by an agency of the United States Government. Neither the United States Government nor any agency thereof, nor any of their employees, makes any warranty, express or implied, or assumes any legal liability or responsibility for the accuracy, completeness, or usefulness of any information, apparatus, product, or process disclosed, or represents that its use would not infringe privately owned rights. Reference herein to any specific commercial product, process, or service by trade name, trademark, manufacturer, or otherwise, does not necessarily constitute or imply its endorsement, recommendation, or favoring by the United States Government or any agency thereof. The views and opinions of authors expressed herein do not necessarily state or reflect those of the United States Government or any agency thereof. 
Nuclear Energy and Fuel Cycle Division

\title{
Fabrication of MiniFuel Compacts for High-Power Irradiation Testing of TRISO Fuel
}

\author{
Tyler J. Gerczak \\ Grant W. Helmreich \\ Christopher A. Hobbs \\ Andrew K. Kercher \\ Ryan Latta
}

Date Published: January 2021

\author{
Prepared by \\ OAK RIDGE NATIONAL LABORATORY \\ Oak Ridge, TN 37831-6283 \\ managed by \\ UT-BATTELLE, LLC \\ for the \\ US DEPARTMENT OF ENERGY \\ under contract DE-AC05-00OR22725
}





\section{CONTENTS}

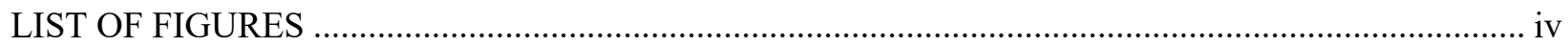

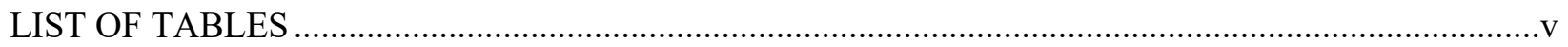

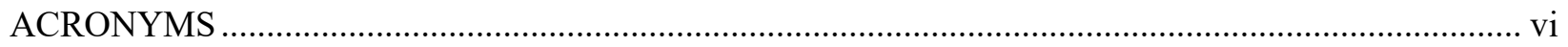

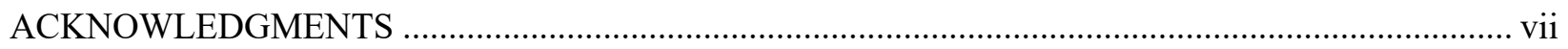

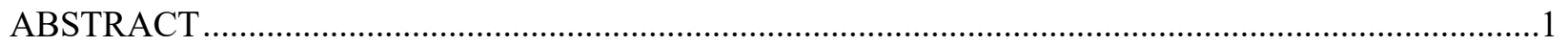

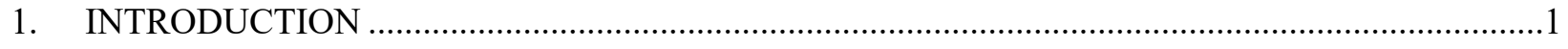

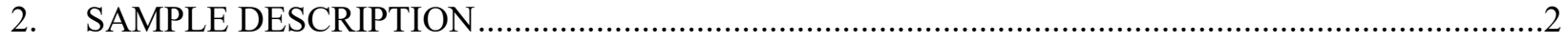

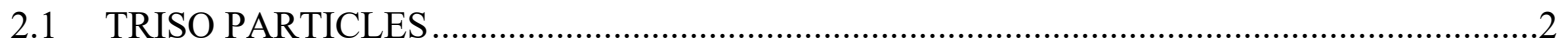

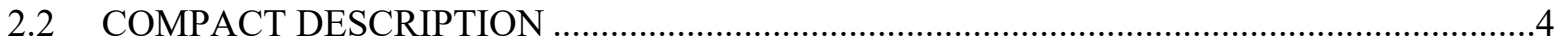

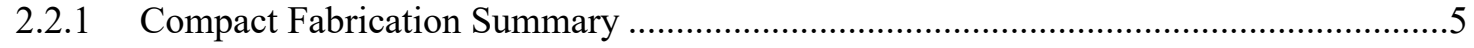

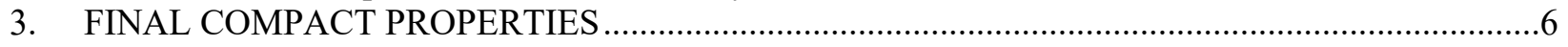

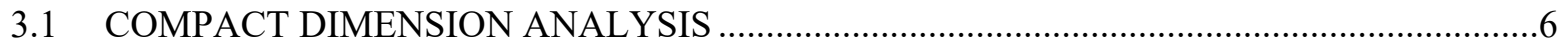

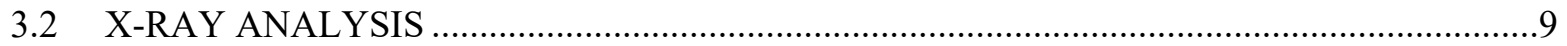

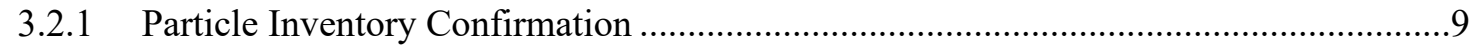

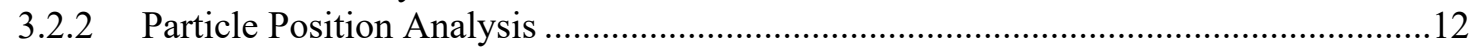

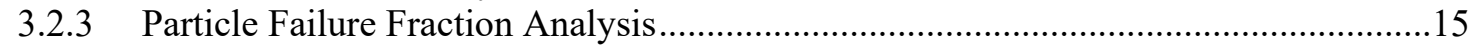

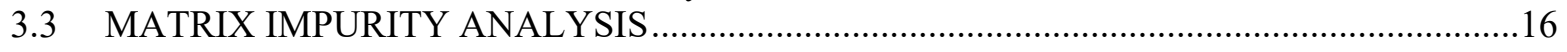

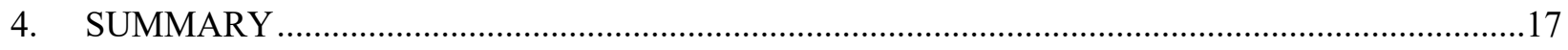

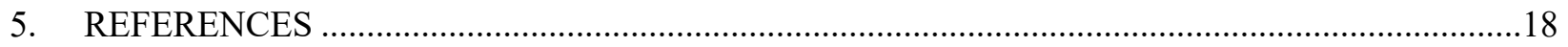

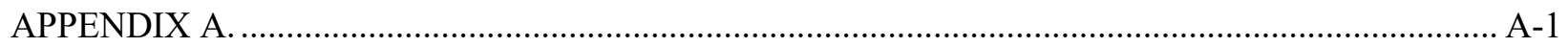

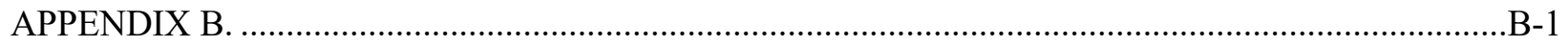

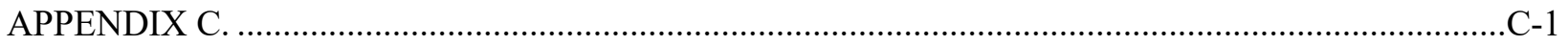




\section{LIST OF FIGURES}

Figure 1. Example of optical image used for kernel compositional analysis (left) and segmented

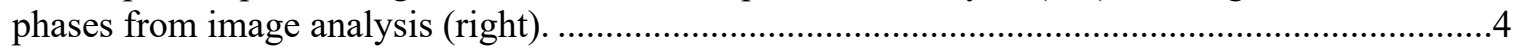

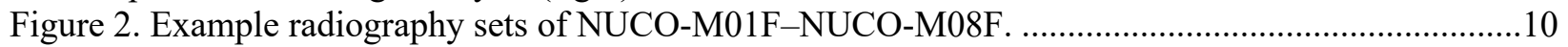

Figure 3. Example radiography sets of LEU09-M08E-LEU09-M12E. ..................................................10

Figure 4. Example radiograph sets of LEU11-M05E-LEU11-M08E...................................................11

Figure 5. Cross-sectional tomogram from 3D tomograph of a compact showing kernels and $\mathrm{SiC}$ layers from several particles. ................................................................................................12

Figure 6. Side view of a 3D representation of an XCT image for a compact showing each particle and its associated region volume within the compact in a unique color.........................................14

Figure 7. Top-down view of a 3D representation of an XCT image for a compact showing each particle and its associated region volume within the compact in a unique color.

Figure 8. Example radiograph of an intact particle (left) and oxidized kernel and missing buffer/IPyC particle (right) after exposure to a burn stage (Gerczak et al. 2016). .......................15

Figure 9. Example of radiography analysis of a 120 particle clutch after $750^{\circ} \mathrm{C}$ for $48 \mathrm{~h}$ under forced air from LEU09-M\#\#E.

Figure A-1. Radiography sets of LEU09-M01E-LEU09-M06E; axial image orientation (left) and $45^{\circ}$ tilt from the axial direction (right)

Figure A-2. Radiography sets of LEU09-M07E-LEU09-M12E; axial image orientation (left) and $45^{\circ}$ tilt from the axial direction (right).

Figure A-3. Radiography sets of LEU09-M13E-LEU09-M18E; axial image orientation (left) and

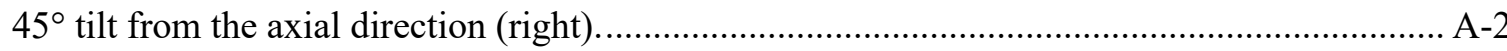

Figure A-4. Radiography sets of LEU09-M19E-LEU09-M24E; axial image orientation (left) and $45^{\circ}$ tilt from the axial direction (right).

Figure A-5. Radiography sets of LEU09-M25E-LEU09-M30E; axial image orientation (left) and

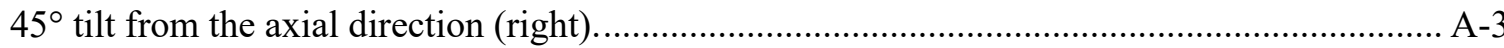

Figure A-6. Radiography sets of LEU09-M31E-LEU09-M36E; axial image orientation (left) and $45^{\circ}$ tilt from the axial direction (right).

Figure A-7. Radiography sets of LEU09-M37E-LEU09-M42E; axial image orientation (left) and $45^{\circ}$ tilt from the axial direction (right).

Figure A-8. Radiography sets of LEU09-M43E-LEU09-M47E; axial image orientation (left) and $45^{\circ}$ tilt from the axial direction (right).

Figure A-9. Radiography sets of LEU11-M01B-LEU11-M04B; axial image orientation (left) and $45^{\circ}$ tilt from the axial direction (right)......

Figure A-10. Radiography sets of LEU11-M05B-LEU11-M08B; axial image orientation (left) and $45^{\circ}$ tilt from the axial direction (right).

Figure A-11. Radiography sets of LEU11-M09B-LEU11-M12B; axial image orientation (left) and $45^{\circ}$ tilt from the axial direction (right).....

Figure A-12. Radiography sets of LEU11-M13B-LEU11-M16B; axial image orientation (left) and $45^{\circ}$ tilt from the axial direction (right).

Figure A-13. Radiography sets of LEU11-M17B-LEU11-M20B; axial image orientation (left) and $45^{\circ}$ tilt from the axial direction (right).

Figure A-14. Radiography sets of LEU11-M21B-LEU11-M22B; axial image orientation (left) and $45^{\circ}$ tilt from the axial direction (right).....

Figure A-15. Radiography sets of NUCO425-08T-M01F- NUCO425-08T-M08F; axial image orientation (left) and $45^{\circ}$ tilt from the axial direction (right)

Figure C-1. Radiograph of burn test clutch \#1 from LEU09-M\#\#E.

Figure C-2. Radiograph of burn test clutch \#2 from LEU09-M\#\#E. C-2 
Figure C-3. Radiograph of burn test clutch \#3 from LEU09-M\#\#E.

Figure C-4. Radiograph of burn test clutch \#1 from LEU09-M\#\#G.

Figure C-5. Radiograph of burn test clutch \#2 from LEU09-M\#\#G.

Figure C-6. Radiograph of burn test clutch \#3 from LEU09-M\#\#G.

Figure C-7. Radiograph of burn test clutch \#4 from LEU09-M\#\#G.

Figure C-8. Radiograph of burn test clutch \#5 from LEU09-M\#\#G.

Figure C-9. Radiograph of burn test clutch \#6 from LEU09-M\#\#G.

Figure C-10. Radiograph of burn test clutch \#7 from LEU09-M\#\#G.

Figure C-11. Radiograph of burn test clutch \#1 from LEU11-M\#\#B.

Figure C-12. Radiograph of burn test clutch \#2 from LEU11-M\#\#B.

C-12

Figure C-13. Radiograph of burn test clutch \#3 from LEU11-M\#\#B.

C-13

\section{LIST OF TABLES}

Table 1. Reported relevant kernel properties of particles used in the fabrication of MiniFuel compacts.

Table 2. Reported relevant particle properties of particles used in the fabrication of MiniFuel compacts.

Table 3. Post-heat-treatment compact properties for NUCO variant compacts (Series M\#\#F). .................6

Table 4. Post-heat-treatment compact properties for LEUCO variant compacts (Series M\#\#E).................7

Table 5. Post-heat-treatment compact properties for LEUCO variant compacts (Series M\#\#G).................8

Table 6. Post-heat-treatment compact properties for $\mathrm{LEUO}_{2}$ variant compacts (Series M\#\#B). .................9

Table 7. Total uranium content of MiniFuel compacts and isotopic breakdown. .....................................11

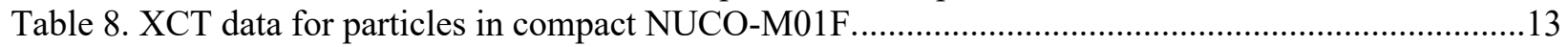

Table 9. Select impurity data for matrix material from MiniFuel compacts. ..........................................17

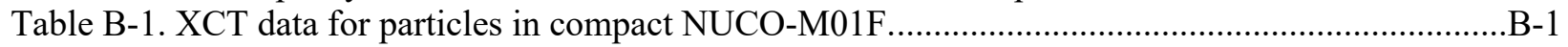

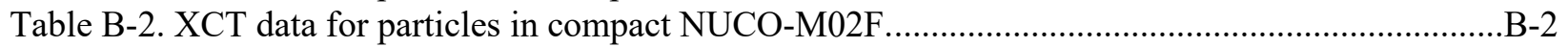

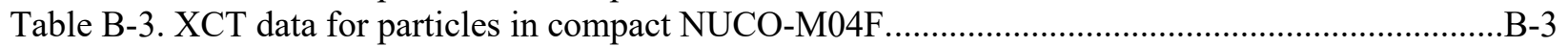

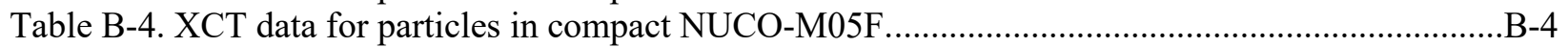

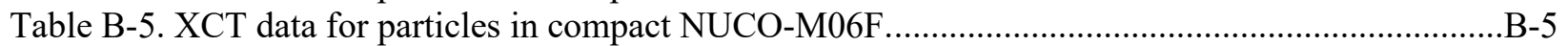

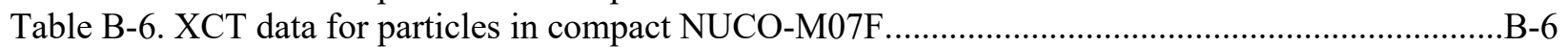

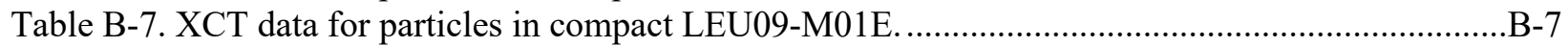

Table B-8. XCT data for particles in compact LEU09-M07E. ...............................................................

Table B-9. XCT data for particles in compact LEU09-M14E..........................................................

Table B-10. XCT data for particles in compact LEU09-M19E...........................................................

Table B-11. XCT data for particles in compact LEU09-M24E. .........................................................

Table B-12. XCT data for particles in compact LEU09-M26E...........................................................12

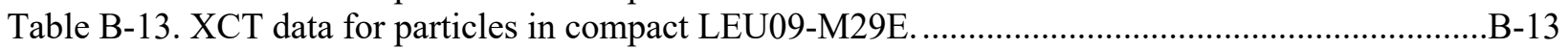

Table B-14. XCT data for particles in compact LEU09-M36E...............................................................

Table B-15. XCT data for particles in compact LEU09-M42E.............................................................15

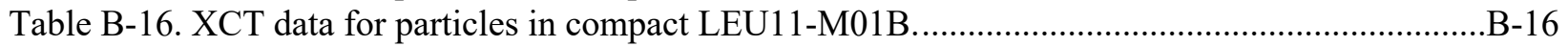

Table B-17. XCT data for particles in compact LEU11-M05B............................................................17

Table B-18. XCT data for particles in compact LEU11-M14B............................................................. 


\section{ACRONYMS}

$\begin{array}{ll}\text { AGR } & \text { Advanced Gas Reactor } \\ \text { DOE } & \text { US Department of Energy } \\ \text { FHR } & \text { Fluoride Salt-Cooled High-Temperature Reactor } \\ \text { HFIR } & \text { High Flux Isotope Reactor } \\ \text { HTGR } & \text { high-temperature gas cooled reactor } \\ \text { KP } & \text { Kairos Power LLC } \\ \text { LEUCO } & \text { low-enriched UCO } \\ \text { LEUO } & \text { low-enriched UO } \\ \text { NUCO } & \text { natural uranium UCO } \\ \text { ORNL } & \text { Oak Ridge National Laboratory } \\ \text { PIE } & \text { postirradiation examination } \\ \text { TRISO } & \text { tristructural isotropic } \\ \text { UCO } & \text { uranium oxide, uranium carbide } \\ \text { UO } 2 & \text { uranium oxide } \\ \text { XCT } & \text { x-ray computed tomography }\end{array}$




\section{ACKNOWLEDGMENTS}

This research was sponsored by the Nuclear Science User Facilities Program of the US Department of Energy (DOE), Office of Nuclear Energy. The report was authored by UT-Battelle under Contract No. DE-AC05-00OR22725 with DOE. 



\begin{abstract}
MiniFuel compacts containing 20 individual tristructural isotropic (TRISO)-coated fuel particles were characterized to provide supporting preirradiation characterization data. The preirradiated characterization data supports irradiation capsule design and safety analysis, as well as downstream postirradiation examination (PIE) for a planned MiniFuel irradiation to explore high particle powers. The analysis included dimensional inspection, x-ray radiography and tomography, as-fabricated defect fraction analysis, and a matrix impurity analysis.
\end{abstract}

\title{
1. INTRODUCTION
}

The performance of tristructural isotropic (TRISO)-coated particle fuel has been established for operating conditions that are typical of high-temperature gas cooled reactors (HTGR) where modern TRISO fuel irradiation experiments have demonstrated failure fractions below design limits at typical HTGR conditions (Demkowicz et al. 2019). The successful demonstration of TRISO fuel for HTGR concepts has led to interest in the TRISO fuel concept for application outside HTGRs due to the robustness of the coated particle fuel architecture. TRISO fuel and TRISO fuel-related concepts are currently under consideration for accident-tolerant light water reactor concepts (Terrani et al. 2012) and molten salt reactor concepts (Brown et al. 2017). These novel designs could explore conditions not typically associated with HTGR operations, such as temperature, fluence, burnup, and power density.

The Kairos Power LLC (KP) Fluoride Salt-Cooled High-Temperature Reactor (FHR) design represents a novel reactor design that uses TRISO fuel particles. The reactor concept leverages a spherical pebble fuel form in a molten salt coolant. This approach effectively couples two Generation IV reactor technologies. The design varies from HTGR concepts in that the properties of the molten salt allow for higher power densities. Also, the potential for the salt coolant to accommodate fission products may support higher particle failure rates compared with traditional HTGRs. The opportunity to operate at higher power densities requires an understanding of particle performance at higher particle powers relative to what has traditionally been explored in HTGR supporting irradiations. An irradiation experiment was developed to explore particle powers at FHR-relevant temperatures for particle powers that bound the operational envelope for very high-power density FHR designs (Latta et al. 2020). The irradiation will be conducted in the High Flux Isotope Reactor (HFIR) at Oak Ridge National Laboratory (ORNL). This experiment is expected to demonstrate TRISO fuel performance at margin conditions to establish the bounding behavior for high particle power operation.

Two distinct comparisons are included in the planned experiment. The first is a comparison between TRISO particles at different particle powers at FHR-relevant operational conditions. Average particle powers are expected to range from $\sim 70-600 \mathrm{~mW}$ and will be tested at two temperatures: 500 and $700^{\circ} \mathrm{C}$. The range in average particle power is influenced by TRISO particle enrichment and will be confirmed through detailed neutronics calculations as part of the irradiation design process. Two different particle types are included: one with kernels with mixed low-enriched uranium oxide and uranium carbide (UCO) kernels and one with natural enriched UCO kernels. The low-enriched particles represent the higher particle power variant, whereas the natural-enriched UCO TRISO particles represent the low particle power range. These two variants are designated as $L E U C O$ and NUCO, respectively. The conditions explored here bound the particle powers explored by the US Department of Energy (DOE) Advanced Gas Reactor Fuel Qualification and Development (AGR) program's second irradiation experiment AGR-2 (Collin 2014). The second comparison is concerned with potential kernel migration at the higher particle powers. Kernel migration has been observed to be a failure mechanism in uranium oxide $\left(\mathrm{UO}_{2}\right)$ kernel particles (Homan et al. 1977). The degree of kernel migration is related to the size of the thermal gradients across the particles (Maki et al., 2007) and kernel migration can be mitigated by adding a 
carbide phase to buffer $\mathrm{CO}_{(\mathrm{g})}$ production (Bullock and Kaae 1983, McMurray et al., 2017). The impact of kernel composition on kernel migration will be explored by comparing low-enriched UCO (LEUCO) and low-enriched $\mathrm{UO}_{2}$ kernel $\left(\mathrm{LEUO}_{2}\right)$ TRISO particles at similar particle powers irradiated at nominally $900^{\circ} \mathrm{C}$.

This report describes the particles and compacted fuel forms to be irradiated and the associated preirradiation characterization completed to support the irradiation design and approval. The irradiation capsule design is discussed in a separate report by Gallagher et al. (2020).

\section{SAMPLE DESCRIPTION}

Three variants were identified to be included in the planned irradiation experiment. The irradiation capsule design leveraged the novel MiniFuel irradiation approach to support the separate effects testing of small fuel volumes (Petrie et al. 2019). A small modification to the irradiation capsule design was incorporated into this experiment to explore small-volume "compacts" to avoid single-point heat conduction and atypical thermal gradients. The compact fabrication process was not optimized beyond providing a 3D heat conduction pathway and ensuring no particles were compromised during fabrication. Particles selected for irradiation were obtained from the existing inventory of particles from the AGR program. These particles were upgraded for roundness via tabling and subsequently compacted into MiniFuel compacts to meet the irradiation capsule design criteria. A total of 20 particles per compact were targeted based on initial neutronics and thermal analysis which was used to optimize the number of particles included in the irradiation to increase particle statistics while limiting particle-to-particle variation in irradiation temperature and particle power. The development and fabrication of the MiniFuel compacts were supported by KP through a Strategic Partnership Program (Gerczak et al. 2020).

\subsection{TRISO PARTICLES}

Particles selected for irradiation were associated with the second AGR irradiation experiment, AGR-2. Because of this pedigree, significant preirradiation characterization has been completed on the kernel and particle properties. Table 1 and Table 2 list the relevant kernel and particle properties for the particles included in this irradiation experiment. Collin (2014) compiled the data, and the source data were provided by BWXT documents (2008a, 2008b, 2008c, 2009) and two documents by Hunn et al. (2008, 2010). The particle lots for the LEUCO (LEU09) and $\mathrm{LEUO}_{2}$ (LEU11) particles were the same as those included in the AGR-2 irradiation experiment, whereas the NUCO (NUCO425-08T) particles were produced before the start of the LEUCO particles coating efforts to establish optimal coating conditions. The NUCO kernels and particles did not receive the same focus as the LEU09 and LEU11 sample sublots because they were not intended to be included in the AGR irradiation campaign. Select values for the NUCO kernels and particles are reported in Table 1 and Table 2, and select values and kernel enrichment were reported previously by Seibert et al. (2019). The mean values reported for NUCO425-08T particle properties in Table 1 and Table 2 were measured to the same standards as the LEU09 and LEU11 particles following AGR program procedures; however, a formal ORNL data package report has not yet been published. Additional NUCO kernel analysis was completed in this effort which focused on kernel phase fraction. 
Table 1. Reported relevant kernel properties of particles used in the fabrication of MiniFuel compacts.

\begin{tabular}{|c|c|c|c|}
\hline Variant & LEUCO & $\mathbf{L E U O}_{2}$ & NUCO \\
\hline Sublot designation & G73I-14-69307 & G73AA-10-69308 & - \\
\hline Diameter $(\mu \mathrm{m})$ & $426.7 \pm 8.8$ & $507.7 \pm 11.9$ & $422.4 \pm 13.8$ \\
\hline Density $\left(\mathrm{Mg} / \mathrm{m}^{3}\right)$ & $10.966 \pm 0.033$ & $10.858 \pm 0.082$ & $10.58 \pm 0.35^{*}$ \\
\hline${ }^{235} \mathrm{U}$ enrichment (wt \%) & $14.029 \pm 0.026$ & $9.600 \pm 0.010$ & 0.71 \\
\hline Carbon/uranium (atomic ratio) & $0.392 \pm 0.002$ & N/A & $0.432 \pm 0.031^{*}$ \\
\hline Oxygen/uranium (atomic ratio) & $1.428 \pm 0.005$ & $2.003 \pm 0.005$ & $1.512 \pm 0.036^{*}$ \\
\hline [Carbon + oxygen]/uranium (atomic ratio) & $1.818 \pm 0.005$ & $\mathrm{~N} / \mathrm{A}$ & $1.944 \pm 0.004^{*}$ \\
\hline Total uranium (wt \%) & $89.463 \pm 0.051$ & N/A & $89.010 \pm 0.001 *$ \\
\hline Sulfur impurity $(\mathrm{ppm}-\mathrm{wt})$ & $365 \pm 12$ & \multirow{2}{*}{$\leq 50$} & N/A \\
\hline Phosphorus impurity $(\mathrm{ppm}-\mathrm{wt})$ & $\leq 50$ & & N/A \\
\hline All other impurities & \multicolumn{2}{|c|}{$\begin{array}{l}\text { Below minimum detection limits and } \\
\text { within specification }\end{array}$} & $\mathrm{N} / \mathrm{A}$ \\
\hline
\end{tabular}

* values measured by automated image analysis

\pm values reported as one standard deviation from the mean

Table 2. Reported relevant particle properties of particles used in the fabrication of MiniFuel compacts.

\begin{tabular}{|l|c|c|c|}
\hline \multicolumn{1}{|c|}{ Variant } & LEUCO & LEUO 2 & NUCO \\
\hline Lot designation & LEU09 & LEU11 & NUCO425-08T \\
\hline Buffer thickness $(\mu \mathrm{m})$ & $98.9 \pm 8.4$ & $97.7 \pm 9.9$ & $106.1 \pm 10.9$ \\
\hline IPyC thickness $(\mu \mathrm{m})$ & $40.4 \pm 2.5$ & $41.9 \pm 3.2$ & $44.4 \pm 2.8$ \\
\hline SiC thickness $(\mu \mathrm{m})$ & $35.2 \pm 1.2$ & $37.5 \pm 1.2$ & $34.9 \pm 1.1$ \\
\hline OPyC thickness $(\mu \mathrm{m})$ & $43.4 \pm 2.9$ & $45.6 \pm 2.4$ & $40.3 \pm 2.9$ \\
\hline Buffer density $\left(\mathrm{Mg} / \mathrm{m}^{3}\right)$ & Not measured & 0.99 & Not measured \\
\hline IPyC density $\left(\mathrm{Mg} / \mathrm{m}^{3}\right)$ & $1.890 \pm 0.011$ & Not measured & Not measured \\
\hline SiC density $\left(\mathrm{Mg} / \mathrm{m}^{3}\right)$ & $3.197 \pm 0.004$ & 3.199 & $3.188 \pm 0.002$ \\
\hline OPyC density $\left(\mathrm{Mg} / \mathrm{m}^{3}\right)$ & $1.907 \pm 0.007$ & $1.884 \pm 0.004$ & $1.903 \pm 0.004$ \\
\hline IPyC anisotropy $(\mathrm{BAF})$ & $1.0349 \pm 0.0012$ & $1.0334 \pm 0.0027$ & $1.0400 \pm 0.0015$ \\
\hline OPyC anisotropy $(\mathrm{BAF})$ & $1.0263 \pm 0.0011$ & $1.0219 \pm 0.0012$ & $1.0276 \pm 0.0010$ \\
\hline SiC sphericity $($ aspect ratio) & $1.037 \pm 0.011$ & $1.034 \pm 0.010$ & Not measured \\
\hline OPyC sphericity $($ aspect ratio) & 1.052 & 1.052 & 1.058 \\
\hline Particle diameter $(\mu \mathrm{m})$ & $873.2 \pm 23$ & $953.0 \pm 28$ & $870.40 \pm 22$ \\
\hline Mean particle mass $(\mathrm{mg})$ & $1.032 \pm 0.003$ & $1.462 \pm 0.005$ & $1.016 \pm 0.002$ \\
\hline
\end{tabular}

\pm values reported as one standard deviation from the mean

The phase fraction data for the carbon/uranium, oxygen/uranium, [carbon + oxygen]/uranium, and total uranium for the NUCO kernels were measured on full TRISO particles, not kernel sublots, as part of the preirradiation characterization effort for MiniFuel compact analysis. Phase fraction and density data were 
measured on a per-kernel basis for approximately 100 NUCO425-08T kernels from full TRISO particles by using previously developed automated image analysis software (Helmreich et al. 2020a). This method segments cross-sectional images of UCO kernels, polished to a known depth, into carbide, oxide, and void phases based on scanning electron microscopy backscatter electron image intensity. These segmented images are analyzed with their known polish-down distances to accurately determine the volumetric fractions for each phase. These volumetric phase fractions can then be converted to atomic or weight fractions and used to calculate overall kernel density if the stoichiometry of the carbide phase is known. Figure 1 shows an example of the analysis process for a single particle where yellow is the oxide phase, teal is the carbide phase, and dark blue is the void space. The carbide phase was assumed to be $90 \%$ uranium dicarbide and 10\% uranium monocarbide. The acquired data are reported in Table 1. Differences in the atomic ratios and kernel density were observed when comparing the data from the LEUCO and NUCO kernels. The reported differences are expected to be associated with the variation in analysis approaches and the fact that one set of analysis was conducted on as-fabricated kernels and the other on coated kernels. Specifically, the lower density was speculated to be resultant from excess void space due to pullout from mechanical sample preparation particularly near the kernel rind. Other artifacts may be resultant from the assumed uranium monocarbide fraction.
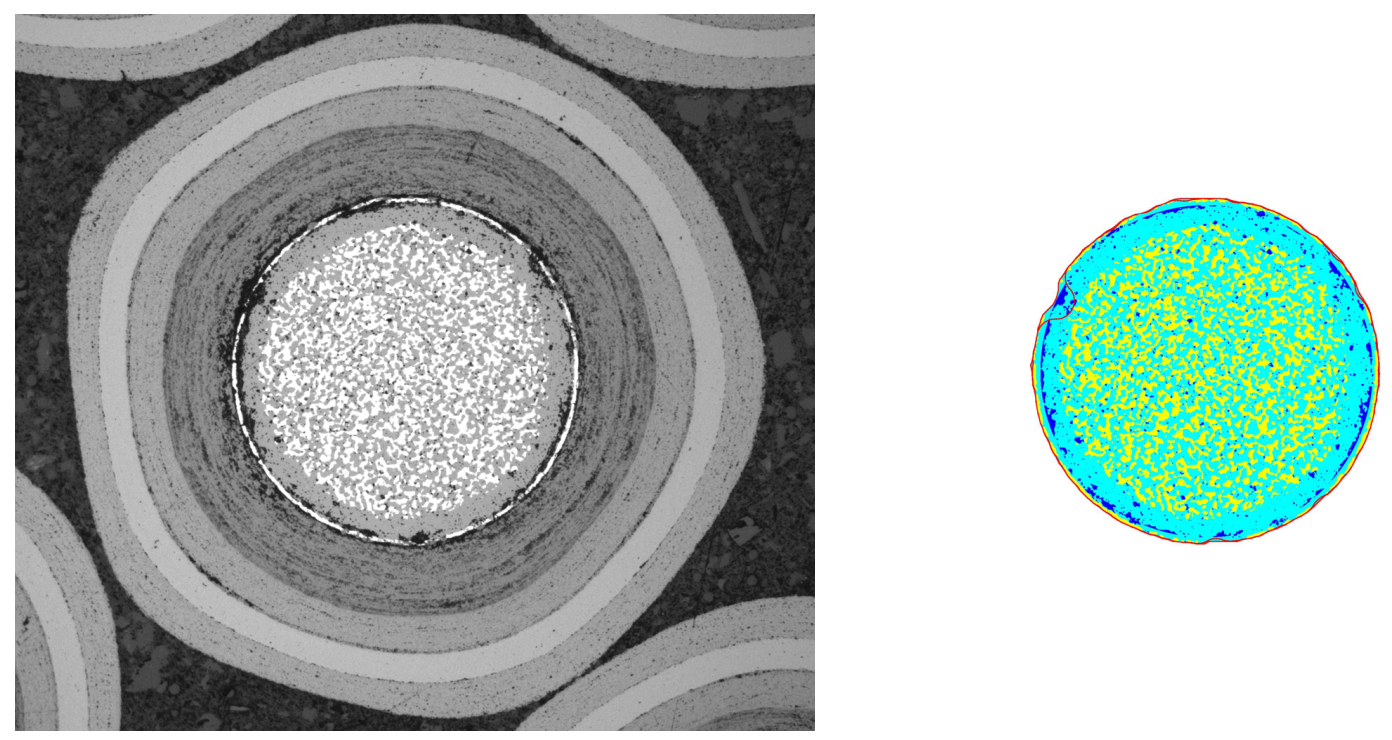

Figure 1. Example of optical image used for kernel compositional analysis (left) and segmented phases from image analysis (right).

\subsection{COMPACT DESCRIPTION}

MiniFuel compacts were produced to provide a 3D heat transfer medium to avoid single-point contact with a sample holder and unintended directional thermal gradients across the particles. KP sponsored the compact fabrication under the strategic partnership project NFE-19-07790 to provide support for "MiniCompact Development for High Power Irradiation testing of TRISO Fuel" in accordance with defined statement of work. The MiniFuel compacts represent the final fuel form that will be inserted into the irradiation capsule build. The preirradiation characterization of the MiniFuel compacts was completed to support the capsule build and approval process, refine thermal models, and support downstream postirradiation examination (PIE) efforts. 


\subsubsection{Compact Fabrication Summary}

The MiniFuel compact fabrication process was reported in the sponsor-controlled report ORNL/SPR2020/1778 (Gerczak et al. 2020), and this section provides a generic overview. The compacting process leveraged techniques and equipment similar to those used to produce compacts for the AGR program (Pappano et al. 2008). The general process consisted of upgrading particles, overcoating particle sublots, pressing green compacts, carbonizing compacts, and performing a final high-temperature heat treatment. Small sublots of particles were riffled out from larger particle volumes to obtain a randomly representative distribution of particles, then upgraded by hand-tabling to remove faceted or other nonideal particles.

Particles were overcoated to predetermined overcoated particle weight targets to form a 20 particle charge for pressing a green compact. Particles were overcoated by using materials and processes that were consistent with compact fabrication from AGR-1 to AGR-4 (Hunn et al. 2010a, Hunn et al. 2010b, Hunn et al. 2014, Pappano et al. 2008, Pappano and Hunn 2008). The matrix was prepared at ORNL. The matrix composition used was similar to the A3-3 refence materials and comprised natural graphite flake, synthetic graphite flake, and a resin binder in a 64:16:20 mass ratio (Gerczak et al. 2020).

A lab-scale overcoater was used to over coat the particles of interest. The system comprised an overcoating drum, ultrasonic atomizer, and syringe pump. A green compact matrix density of $\sim 1.45 \mathrm{~g} / \mathrm{cm}^{3}$ was targeted to meet the final matrix density targets of $1.22-1.34 \mathrm{~g} / \mathrm{cm}^{3}$. This range in final density represents the range in densities found in the AGR-1 irradiation experiment (Hunn et al. 2012). The higher green density accounts for the resin binder volatilization during carbonization and hightemperature heat treatment which leads to a reduction in density after the carbonization step. The green compact weight was used to target average overcoated particle weight and the ultimate 20 particle charge weight.

Overcoated particles were separated into separate bins based on particle diameter by using a roller micrometer. The average particle weight per bin was measured and used to produce 20 particle charges for pressing MiniFuel compacts. Pressing was conducted by using a Promess Inc. automated servo-driven mechanical press and a custom single-acting die and punch set. Pressing was done at a die temperature of $60^{\circ} \mathrm{C}$, and the press height was controlled to fabricate reproducible compact thicknesses. A peak pressing force limit was set at $200 \mathrm{lb}$, which reflects $\sim 7,800 \mathrm{psi}$. This force exceeds the compact pressing pressure of $\sim 2,700$ psi explored in the AGR program and the maximum peak forces measured during pressing of MiniFuel compacts were $\sim 2,445$ psi.

Green compacts were then subjected to carbonization and a heat-treatment schedule similar to those used to fabricate AGR-1 through AGR-4 compacts (AGR-COMP-SOP-05 Rev. 1 and AGR-CHAR-SOG-27). The carbonization step involved thermal exposure at $\sim 950^{\circ} \mathrm{C}$ for $1 \mathrm{~h}$ under flowing inert gas. This process partially carbonizes the resin binder and drives out volatile components. After carbonization, the MiniFuel compacts were subjected to a high-temperature heat treatment at $1,800^{\circ} \mathrm{C}$ for $\sim 1 \mathrm{~h}$. This step drives out impurity elements and further carbonizes the resin. Afterward, fabrication compacts were subjected to a series of characterizations to provide data relevant to the irradiation planning and downstream PIE. 


\section{FINAL COMPACT PROPERTIES}

\subsection{COMPACT DIMENSION ANALYSIS}

An analysis was performed on all produced compacts to measure the weight and dimension and to calculate density. The methods for measuring these properties were based on established procedures from the AGR program (AGR-CHAR-DAM-42, Rev. 1) that were modified slightly due to the unique geometry of these samples. The compact dimensions were measured using a Mitotuyo digital caliper to measure the diameter and a Starrett digital height gauge to measure the compact thickness. The compact weight was measured by using a Mettler Toledo digital balance. The matrix density was calculated by isolating the matrix volume, and the weight was determined by subtracting the volume and weight of 20 particles by using the average particle properties in Table 2 . The calculated matrix density was a primary down-selection criterion for identifying compacts for irradiation and archiving samples. Specifically, outlier densities were excluded to control for this property. Other down-selection criteria are discussed in Section 3.2.1. Compacts that were not selected for irradiation or archive were subjected to burn testing for as-fabricated particle defect analysis (Section 3.2.3). Most compacts from the LEUCO-M\#\#G series were subjected to burn testing because these were produced specifically to supply compacts for defect analysis. Select compacts, designated as "Yes" in the XCT column of Table 3-Table 6, were subjected to 3D x-ray computed tomography (XCT) analysis to support downstream PIE and the refinement of thermal models (Section 3.2.2).

Table 3. Post-heat-treatment compact properties for NUCO variant compacts (Series M\#\#F).

\begin{tabular}{|c|c|c|c|c|c|c|c|}
\hline Compact ID & $\begin{array}{c}\text { Weight } \\
(\mathbf{g})\end{array}$ & $\begin{array}{c}\text { Height } \\
(\mathbf{m m})\end{array}$ & $\begin{array}{c}\text { Dia. } \\
\mathbf{( m m})\end{array}$ & $\begin{array}{c}\mathbf{\rho} \\
\left(\mathbf{g} / \mathbf{c m}^{\mathbf{3}}\right)\end{array}$ & $\begin{array}{c}\text { Matrix } \\
\mathbf{\rho} \\
\left(\mathbf{g} / \mathbf{c m}^{\mathbf{3}}\right)\end{array}$ & Status & XCT \\
\hline NUCO-M01F & 0.0627 & 2.33 & 4.55 & 1.6550 & 1.3690 & Archive/Irradiation & Yes \\
\hline NUCO-M02F & 0.0598 & 2.31 & 4.54 & 1.5991 & 1.2959 & Archive/Irradiation & Yes \\
\hline NUCO-M03F & 0.0591 & 2.30 & 4.55 & 1.5803 & 1.2729 & Archive/Irradiation & No \\
\hline NUCO-M04F & 0.0601 & 2.31 & 4.55 & 1.6001 & 1.2987 & Archive/Irradiation & Yes \\
\hline NUCO-M05F & 0.0594 & 2.32 & 4.55 & 1.5747 & 1.2692 & Archive/Irradiation & Yes \\
\hline NUCO-M06F & 0.0592 & 2.31 & 4.56 & 1.5692 & 1.2626 & Archive/Irradiation & Yes \\
\hline NUCO-M07F & 0.0597 & 2.30 & 4.54 & 1.6034 & 1.2995 & Archive/Irradiation & Yes \\
\hline NUCO-M08F & 0.0607 & 2.30 & 4.54 & 1.6303 & 1.3325 & Archive/Irradiation & No \\
\hline Min & 0.0591 & 2.30 & 4.54 & 1.5692 & 1.2626 & - & - \\
\hline Max & 0.0627 & 2.33 & 4.56 & 1.6550 & 1.3690 & - & - \\
\hline Mean & 0.0601 & 2.31 & 4.55 & 1.6015 & 1.3001 & - & - \\
\hline Standard dev. & 0.0012 & 0.01 & 0.01 & 0.0291 & 0.0357 & - & - \\
\hline
\end{tabular}


Table 4. Post-heat-treatment compact properties for LEUCO variant compacts (Series M\#\#E).

\begin{tabular}{|c|c|c|c|c|c|c|c|}
\hline Compact ID & $\begin{array}{c}\text { Weight } \\
\text { (g) }\end{array}$ & $\begin{array}{c}\text { Height } \\
(\mathbf{m m})\end{array}$ & $\begin{array}{l}\text { Dia. } \\
(\mathbf{m m})\end{array}$ & $\underset{\left(\mathrm{g} / \mathrm{cm}^{3}\right)}{\rho}$ & $\begin{array}{c}\text { Matrix } \\
\rho \\
\left(\mathrm{g} / \mathrm{cm}^{3}\right)\end{array}$ & Status & XCT \\
\hline LEU09-M01E & 0.0626 & 2.37 & 4.59 & 1.5963 & 1.3013 & Archive/Irradiation & Yes \\
\hline LEU09-M02E & 0.0614 & 2.36 & 4.58 & 1.5792 & 1.2774 & Archive/Irradiation & No \\
\hline LEU09-M03E & 0.0618 & 2.37 & 4.58 & 1.5828 & 1.2833 & Archive/Irradiation & No \\
\hline LEU09-M04E & 0.0631 & 2.36 & 4.60 & 1.6088 & 1.3166 & Burn test & No \\
\hline LEU09-M05E & 0.0620 & 2.36 & 4.58 & 1.5946 & 1.2962 & Burn test & No \\
\hline LEU09-M06E & 0.0611 & 2.35 & 4.58 & 1.5782 & 1.2746 & Archive/Irradiation & No \\
\hline LEU09-M07E & 0.0615 & 2.34 & 4.57 & 1.6023 & 1.3008 & Archive/Irradiation & Yes \\
\hline LEU09-M08E & 0.0621 & 2.35 & 4.57 & 1.6110 & 1.3131 & Archive/Irradiation & No \\
\hline LEU09-M09E & 0.0604 & 2.34 & 4.56 & 1.5805 & 1.2726 & Archive/Irradiation & No \\
\hline LEU09-M10E & 0.0628 & 2.36 & 4.58 & 1.6152 & 1.3213 & Archive/Irradiation & No \\
\hline LEU09-M11E & 0.0633 & 2.37 & 4.58 & 1.6212 & 1.3301 & Archive/Irradiation & No \\
\hline LEU09-M12E & 0.0617 & 2.34 & 4.59 & 1.5935 & 1.2933 & Burn test & No \\
\hline LEU09-M13E & 0.0624 & 2.33 & 4.59 & 1.6185 & 1.3223 & Archive/Irradiation & No \\
\hline LEU09-M14E & 0.0622 & 2.36 & 4.58 & 1.5998 & 1.3025 & Archive/Irradiation & Yes \\
\hline LEU09-M15E & 0.0611 & 2.34 & 4.58 & 1.5849 & 1.2812 & Burn test & No \\
\hline LEU09-M16E & 0.0620 & 2.34 & 4.57 & 1.6153 & 1.3167 & Archive/Irradiation & No \\
\hline LEU09-M17E & 0.0615 & 2.34 & 4.59 & 1.5883 & 1.2870 & Archive/Irradiation & No \\
\hline LEU09-M18E & 0.0625 & 2.35 & 4.57 & 1.6214 & 1.3257 & Archive/Irradiation & No \\
\hline LEU09-M19E & 0.0620 & 2.35 & 4.57 & 1.6084 & 1.3099 & Archive/Irradiation & Yes \\
\hline LEU09-M20E & 0.0624 & 2.34 & 4.58 & 1.6186 & 1.3224 & Archive/Irradiation & No \\
\hline LEU09-M21E & 0.0629 & 2.35 & 4.58 & 1.6247 & 1.3313 & Archive/Irradiation & No \\
\hline LEU09-M22E & 0.0609 & 2.34 & 4.58 & 1.5797 & 1.2749 & Archive/Irradiation & No \\
\hline LEU09-M23E & 0.0606 & 2.33 & 4.57 & 1.5856 & 1.2789 & Burn test & No \\
\hline LEU09-M24E & 0.0618 & 2.34 & 4.58 & 1.6031 & 1.3034 & Archive/Irradiation & Yes \\
\hline LEU09-M25E & 0.0614 & 2.32 & 4.58 & 1.6064 & 1.3043 & Archive/Irradiation & No \\
\hline LEU09-M26E & 0.0616 & 2.33 & 4.57 & 1.6118 & 1.3109 & Archive/Irradiation & Yes \\
\hline LEU09-M27E & 0.0605 & 2.34 & 4.57 & 1.5762 & 1.2690 & Archive/Irradiation & No \\
\hline LEU09-M28E & 0.0636 & 2.35 & 4.59 & 1.6356 & 1.3462 & Burn test & No \\
\hline LEU09-M29E & 0.0626 & 2.36 & 4.58 & 1.6101 & 1.3150 & Archive/Irradiation & Yes \\
\hline LEU09-M30E & 0.0635 & 2.36 & 4.60 & 1.6190 & 1.3290 & Burn test & No \\
\hline LEU09-M31E & 0.0605 & 2.32 & 4.57 & 1.5898 & 1.2824 & Burn test & No \\
\hline LEU09-M32E & 0.0613 & 2.33 & 4.58 & 1.5969 & 1.2943 & Archive/Irradiation & No \\
\hline LEU09-M33E & 0.0612 & 2.33 & 4.57 & 1.6013 & 1.2981 & Burn test & No \\
\hline LEU09-M34E & 0.0628 & 2.33 & 4.59 & 1.6289 & 1.3349 & Archive/Irradiation & No \\
\hline LEU09-M35E & 0.0610 & 2.32 & 4.57 & 1.6029 & 1.2985 & Archive/Irradiation & No \\
\hline LEU09-M36E & 0.0623 & 2.35 & 4.59 & 1.6022 & 1.3054 & Archive/Irradiation & Yes \\
\hline LEU09-M37E & 0.0624 & 2.35 & 4.59 & 1.6047 & 1.3086 & Burn test & No \\
\hline LEU09-M38E & 0.0612 & 2.35 & 4.58 & 1.5807 & 1.2777 & Archive/Irradiation & No \\
\hline LEU09-M39E & 0.0612 & 2.34 & 4.58 & 1.5875 & 1.2844 & Archive/Irradiation & No \\
\hline LEU09-M40E & 0.0611 & 2.34 & 4.59 & 1.5780 & 1.2744 & Burn test & No \\
\hline LEU09-M41E & 0.0607 & 2.34 & 4.58 & 1.5745 & 1.2686 & Archive/Irradiation & No \\
\hline LEU09-M42E & 0.0623 & 2.34 & 4.58 & 1.6160 & 1.3192 & Archive/Irradiation & Yes \\
\hline LEU09-M43E & 0.0606 & 2.37 & 4.58 & 1.5520 & 1.2459 & Burn test & No \\
\hline LEU09-M44E & 0.0609 & 2.37 & 4.58 & 1.5597 & 1.2553 & Burn test & No \\
\hline LEU09-M45E & 0.0603 & 2.36 & 4.56 & 1.5645 & 1.2563 & Archive/Irradiation & No \\
\hline LEU09-M46E & 0.0605 & 2.34 & 4.58 & 1.5693 & 1.2622 & Archive/Irradiation & No \\
\hline LEU09-M47E & 0.0600 & 2.34 & 4.58 & 1.5564 & 1.2464 & Burn test & No \\
\hline Min & 0.0600 & 2.32 & 4.56 & 1.5520 & 1.2459 & - & - \\
\hline $\operatorname{Max}$ & 0.0636 & 2.37 & 4.60 & 1.6356 & 1.3462 & - & - \\
\hline Mean & 0.0617 & 2.35 & 4.580 & 1.5965 & 1.2963 & - & - \\
\hline Standard dev. & 0.0009 & 0.014 & 0.009 & 0.0200 & 0.0246 & - & - \\
\hline
\end{tabular}


Table 5. Post-heat-treatment compact properties for LEUCO variant compacts (Series M\#\#G).

\begin{tabular}{|c|c|c|c|c|c|c|c|}
\hline Compact ID & $\begin{array}{c}\text { Weight } \\
\text { (g) }\end{array}$ & $\begin{array}{c}\text { Height } \\
\text { (mm) }\end{array}$ & $\begin{array}{l}\text { Dia. } \\
(\mathbf{m m})\end{array}$ & $\underset{\left(\mathrm{g} / \mathrm{cm}^{3}\right)}{\rho}$ & $\begin{array}{c}\text { Matrix } \\
\rho \\
\left(\mathrm{g} / \mathrm{cm}^{3}\right)\end{array}$ & Status & XCT \\
\hline LEU09-M02G & 0.0615 & 2.360 & 4.58 & 1.5818 & 1.2805 & Burn test & No \\
\hline LEU09-M03G & 0.0640 & 2.361 & 4.60 & 1.6332 & 1.3460 & Archive/Irradiation & No \\
\hline LEU09-M04G & 0.0612 & 2.361 & 4.60 & 1.5620 & 1.2593 & Burn test & No \\
\hline LEU09-M05G & 0.0635 & 2.371 & 4.59 & 1.6160 & 1.3260 & Burn test & No \\
\hline LEU09-M06G & 0.0632 & 2.354 & 4.59 & 1.6225 & 1.3309 & Burn test & No \\
\hline LEU09-M07G & 0.0631 & 2.358 & 4.59 & 1.6146 & 1.3224 & Burn test & No \\
\hline LEU09-M08G & 0.0625 & 2.358 & 4.58 & 1.6065 & 1.3109 & Burn test & No \\
\hline LEU09-M09G & 0.0617 & 2.368 & 4.58 & 1.5813 & 1.2813 & Burn test & No \\
\hline LEU09-M10G & 0.0641 & 2.359 & 4.60 & 1.6376 & 1.3510 & Archive/Irradiation & No \\
\hline LEU09-M11G & 0.0635 & 2.363 & 4.60 & 1.6146 & 1.3247 & Burn test & No \\
\hline LEU09-M12G & 0.0632 & 2.373 & 4.60 & 1.6026 & 1.3110 & Burn test & No \\
\hline LEU09-M13G & 0.0641 & 2.375 & 4.59 & 1.6332 & 1.3466 & Burn test & No \\
\hline LEU09-M14G & 0.0627 & 2.371 & 4.59 & 1.6005 & 1.3061 & Burn test & No \\
\hline LEU09-M15G & 0.0638 & 2.359 & 4.59 & 1.6345 & 1.3461 & Burn test & No \\
\hline LEU09-M16G & 0.0640 & 2.354 & 4.60 & 1.6359 & 1.3487 & Burn test & No \\
\hline LEU09-M17G & 0.0636 & 2.359 & 4.57 & 1.6412 & 1.3518 & Burn test & No \\
\hline LEU09-M18G & 0.0631 & 2.356 & 4.58 & 1.6278 & 1.3356 & Burn test & No \\
\hline LEU09-M19G & 0.0642 & 2.359 & 4.60 & 1.6397 & 1.3536 & Burn test & No \\
\hline LEU09-M20G & 0.0623 & 2.358 & 4.59 & 1.5990 & 1.3023 & Burn test & No \\
\hline LEU09-M21G & 0.0641 & 2.354 & 4.59 & 1.6433 & 1.3566 & Burn test & No \\
\hline LEU09-M22G & 0.0628 & 2.356 & 4.58 & 1.6156 & 1.3217 & Burn test & No \\
\hline LEU09-M23G & 0.0629 & 2.361 & 4.60 & 1.6056 & 1.3123 & Burn test & No \\
\hline LEU09-M24G & 0.0644 & 2.363 & 4.59 & 1.6473 & 1.3623 & Archive/Irradiation & No \\
\hline LEU09-M25G & 0.0625 & 2.358 & 4.57 & 1.6138 & 1.3181 & Burn test & No \\
\hline LEU09-M26G & 0.0624 & 2.359 & 4.58 & 1.6035 & 1.3073 & Burn test & No \\
\hline LEU09-M27G & 0.0632 & 2.377 & 4.59 & 1.6045 & 1.3129 & Burn test & No \\
\hline LEU09-M28G & 0.0628 & 2.354 & 4.59 & 1.6148 & 1.3209 & Burn test & No \\
\hline LEU09-M29G & 0.0645 & 2.380 & 4.59 & 1.6404 & 1.3559 & Burn test & No \\
\hline LEU09-M30G & 0.0625 & 2.334 & 4.59 & 1.6183 & 1.3227 & Burn test & No \\
\hline LEU09-M31G & 0.0627 & 2.378 & 4.57 & 1.6051 & 1.3106 & Burn test & No \\
\hline LEU09-M32G & 0.0641 & 2.375 & 4.60 & 1.6264 & 1.3397 & Burn test & No \\
\hline LEU09-M33G & 0.0630 & 2.363 & 4.58 & 1.6183 & 1.3255 & Burn test & No \\
\hline LEU09-M34G & 0.0641 & 2.366 & 4.59 & 1.6352 & 1.3485 & Burn test & No \\
\hline LEU09-M35G & 0.0637 & 2.360 & 4.59 & 1.6334 & 1.3445 & Burn test & No \\
\hline LEU09-M36G & 0.0630 & 2.348 & 4.59 & 1.6218 & 1.3290 & Burn test & No \\
\hline LEU09-M37G & 0.0633 & 2.364 & 4.58 & 1.6229 & 1.3318 & Burn test & No \\
\hline LEU09-M38G & 0.0612 & 2.359 & 4.58 & 1.5727 & 1.2698 & Burn test & No \\
\hline LEU09-M39G & 0.0621 & 2.357 & 4.59 & 1.5920 & 1.2942 & Burn test & No \\
\hline LEU09-M40G & 0.0643 & 2.384 & 4.59 & 1.6324 & 1.3468 & Burn test & No \\
\hline LEU09-M41G & 0.0621 & 2.369 & 4.56 & 1.6028 & 1.3049 & Burn test & No \\
\hline LEU09-M42G & 0.0620 & 2.377 & 4.57 & 1.5876 & 1.2893 & Burn test & No \\
\hline LEU09-M43G & 0.0611 & 2.366 & 4.59 & 1.5632 & 1.2598 & Burn test & No \\
\hline LEU09-M44G & 0.0623 & 2.350 & 4.57 & 1.6160 & 1.3192 & Burn test & No \\
\hline LEU09-M45G & 0.0613 & 2.342 & 4.58 & 1.5913 & 1.2887 & Burn test & No \\
\hline LEU09-M46G & 0.0627 & 2.354 & 4.59 & 1.6074 & 1.3129 & Burn test & No \\
\hline Min & 0.0611 & 2.334 & 4.56 & 1.5620 & 1.2593 & - & - \\
\hline $\operatorname{Max}$ & 0.0645 & 2.384 & 4.60 & 1.6473 & 1.3623 & - & - \\
\hline Mean & 0.0630 & 2.3619 & 4.587 & 1.6138 & 1.3209 & - & - \\
\hline Standard dev. & 0.0009 & 0.010 & 0.01 & 0.0213 & 0.0263 & - & - \\
\hline
\end{tabular}


Table 6. Post-heat-treatment compact properties for $\mathrm{LEUO}_{2}$ variant compacts (Series M\#\#B).

\begin{tabular}{|c|c|c|c|c|c|c|c|}
\hline Compact ID & $\begin{array}{c}\text { Weight } \\
\text { (g) }\end{array}$ & $\begin{array}{c}\text { Height } \\
\text { (mm) }\end{array}$ & $\begin{array}{l}\text { Dia. } \\
(\mathrm{mm})\end{array}$ & $\underset{\left(\mathrm{g} / \mathrm{cm}^{3}\right)}{\rho}$ & $\begin{array}{c}\text { Matrix } \\
\rho \\
\left(\mathrm{g} / \mathrm{cm}^{3}\right)\end{array}$ & Status & XCT \\
\hline LEU11-M01B & 0.0674 & 2.32 & 4.58 & 1.7634 & 1.3087 & Archive/Irradiation & Yes \\
\hline LEU11-M02B & 0.0693 & 2.33 & 4.58 & 1.8053 & 1.3662 & Burn test & No \\
\hline LEU11-M03B & 0.0693 & 2.33 & 4.59 & 1.7975 & 1.3584 & Burn test & No \\
\hline LEU11-M04B & 0.0654 & 2.32 & 4.58 & 1.7111 & 1.2401 & Burn test & No \\
\hline LEU11-M05B & 0.0671 & 2.31 & 4.58 & 1.7632 & 1.3058 & Archive/Irradiation & Yes \\
\hline LEU11-M06B & 0.0689 & 2.33 & 4.58 & 1.7949 & 1.3525 & Burn test & No \\
\hline LEU11-M07B & 0.0670 & 2.32 & 4.58 & 1.7529 & 1.2950 & Burn test & No \\
\hline LEU11-M08B & 0.0670 & 2.33 & 4.58 & 1.7454 & 1.2877 & Archive/Irradiation & No \\
\hline LEU11-M09B & 0.0670 & 2.33 & 4.58 & 1.7454 & 1.2877 & Archive/Irradiation & No \\
\hline LEU11-M10B & 0.0662 & 2.33 & 4.58 & 1.7246 & 1.2604 & Archive/Irradiation & No \\
\hline LEU11-M11B & 0.0661 & 2.32 & 4.58 & 1.7294 & 1.2641 & Burn test & No \\
\hline LEU11-M12B & 0.0674 & 2.33 & 4.58 & 1.7558 & 1.3014 & Archive/Irradiation & No \\
\hline LEU11-M13B & 0.0683 & 2.33 & 4.59 & 1.7715 & 1.3245 & Archive/Irradiation & No \\
\hline LEU11-M14B & 0.0668 & 2.33 & 4.58 & 1.7402 & 1.2809 & Archive/Irradiation & Yes \\
\hline LEU11-M15B & 0.0668 & 2.31 & 4.58 & 1.7553 & 1.2955 & Archive/Irradiation & No \\
\hline LEU11-M16B & 0.0659 & 2.34 & 4.57 & 1.7169 & 1.2504 & Burn test & No \\
\hline LEU11-M17B & 0.0651 & 2.32 & 4.58 & 1.7032 & 1.2298 & Burn test & No \\
\hline LEU11-M18B & 0.0659 & 2.32 & 4.58 & 1.7242 & 1.2573 & Burn test & No \\
\hline LEU11-M19B & 0.0654 & 2.32 & 4.59 & 1.7036 & 1.2331 & Burn test & No \\
\hline LEU11-M20B & 0.0662 & 2.32 & 4.59 & 1.7245 & 1.2603 & Archive/Irradiation & No \\
\hline LEU11-M21B & 0.0647 & 2.31 & 4.58 & 1.7001 & 1.2230 & Burn test & No \\
\hline LEU11-M22B & 0.0648 & 2.34 & 4.56 & 1.6957 & 1.2198 & Burn test & No \\
\hline Min & 0.0647 & 2.31 & 4.56 & 1.6957 & 1.2198 & - & - \\
\hline Max & 0.0693 & 2.34 & 4.59 & 1.8053 & 1.3662 & - & - \\
\hline Mean & 0.0667 & 2.325 & 4.580 & 1.7420 & 1.2819 & - & - \\
\hline Standard dev. & 0.0013 & 0.009 & 0.007 & 0.0322 & 0.0430 & - & - \\
\hline
\end{tabular}

\subsection{X-RAY ANALYSIS}

\subsubsection{Particle Inventory Confirmation}

All compacts considered for inclusion in the planned irradiation were screened to confirm the total number of particles and identify any potential defects or irregularities. Screening was completed by x-ray radiography via a Zeiss Versa $520 \mathrm{XCT}$ instrument. Radiographs were taken along the axial direction of the right cylindrical compacts and at a $45^{\circ}$ tilt from the axial direction. These two angles provided multiple perspectives to accurately count how many particles were in each compact. Example radiograph sets are show in Figure 2, Figure 3, and Figure 4 for NUCO, LEUCO, and $\mathrm{LEUO}_{2}$ compacts, respectively. The LEU09-M\#\#G series of compacts was not subjected to radiography analysis because they were provided exclusively for defect fraction analysis (Section 3.2.3). All radiography pairs used for particle count analysis are presented in Appendix A. 


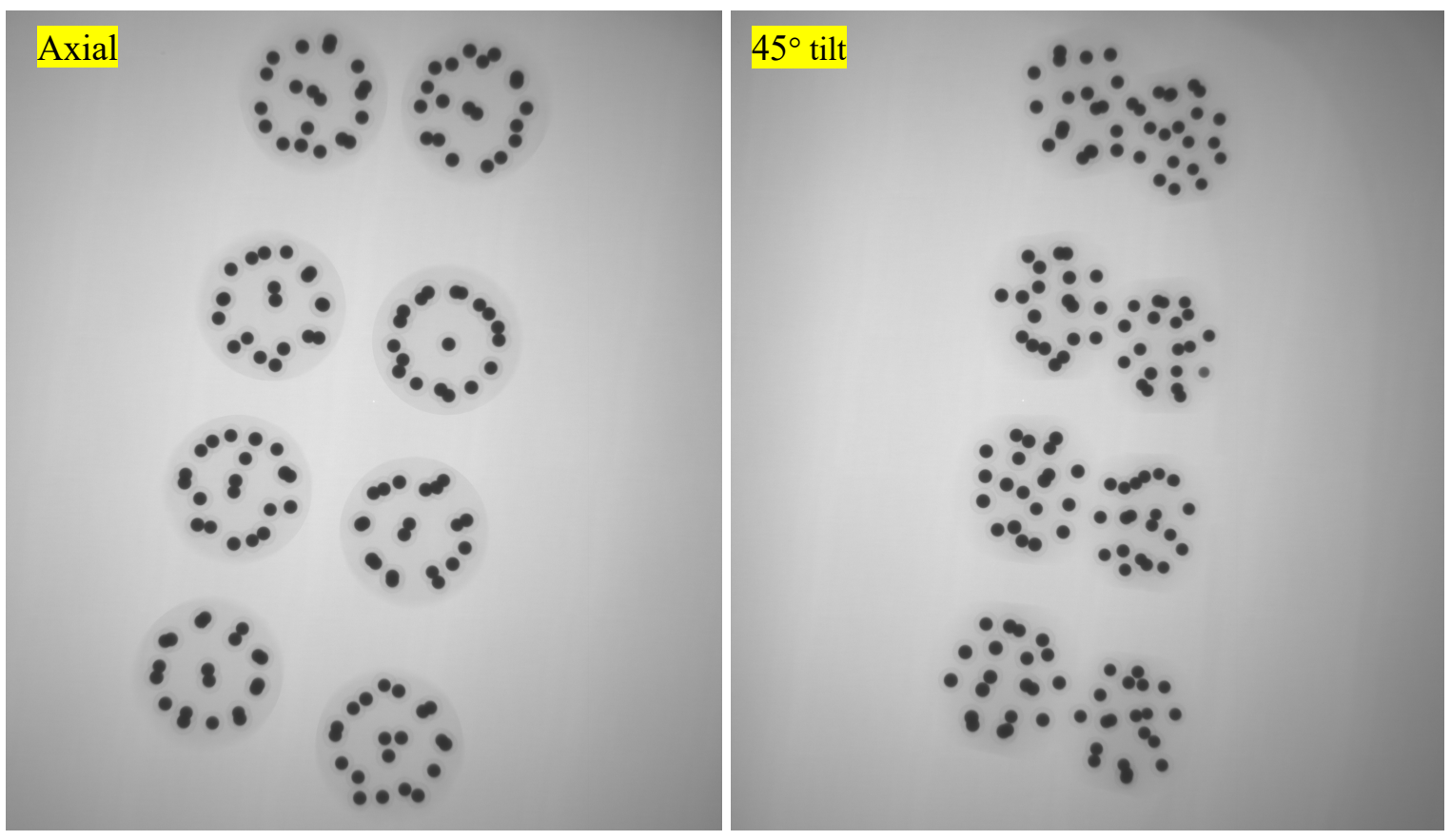

Figure 2. Example radiography sets of NUCO-M01F-NUCO-M08F.
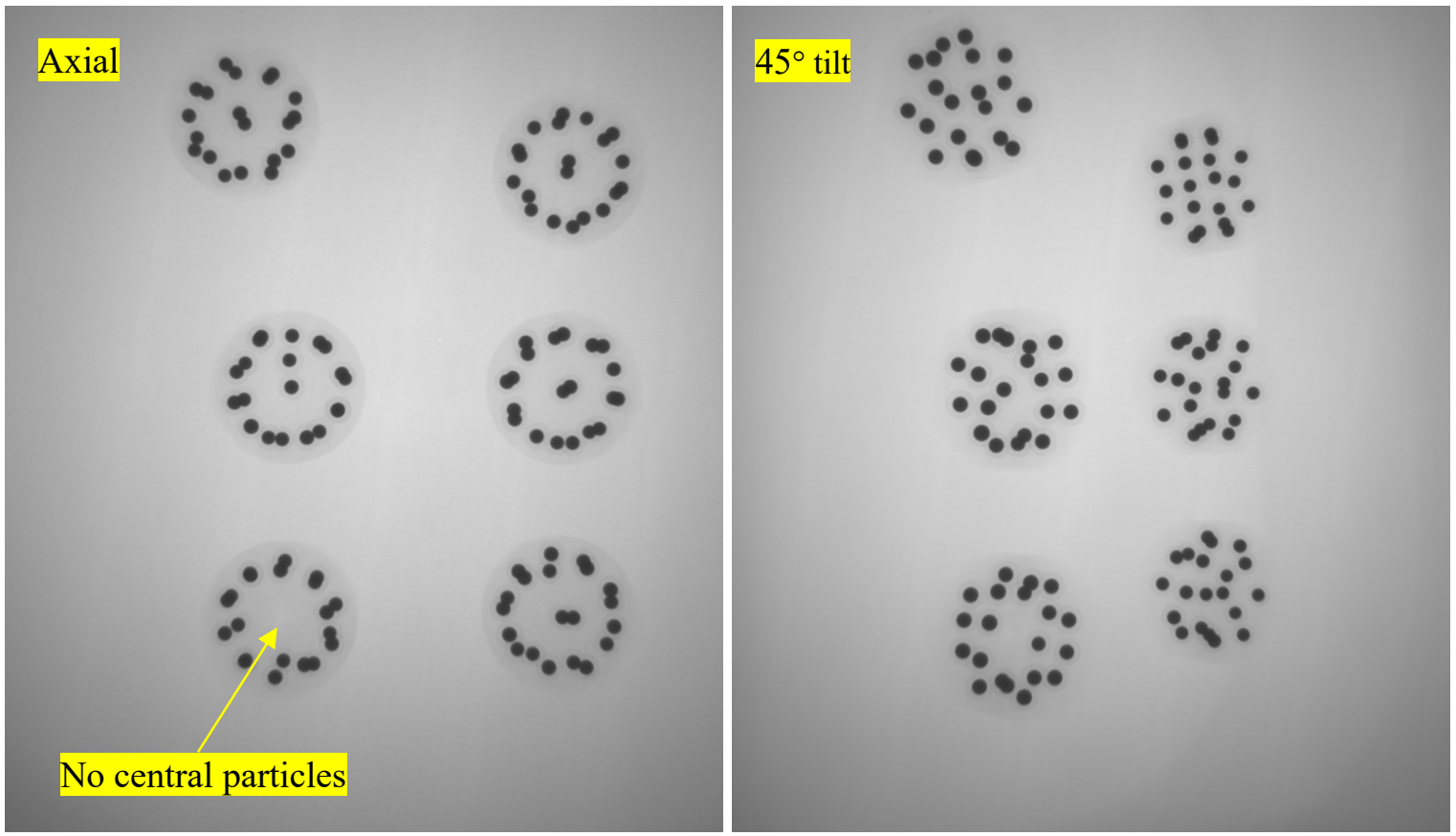

Figure 3. Example radiography sets of LEU09-M08E-LEU09-M12E. 

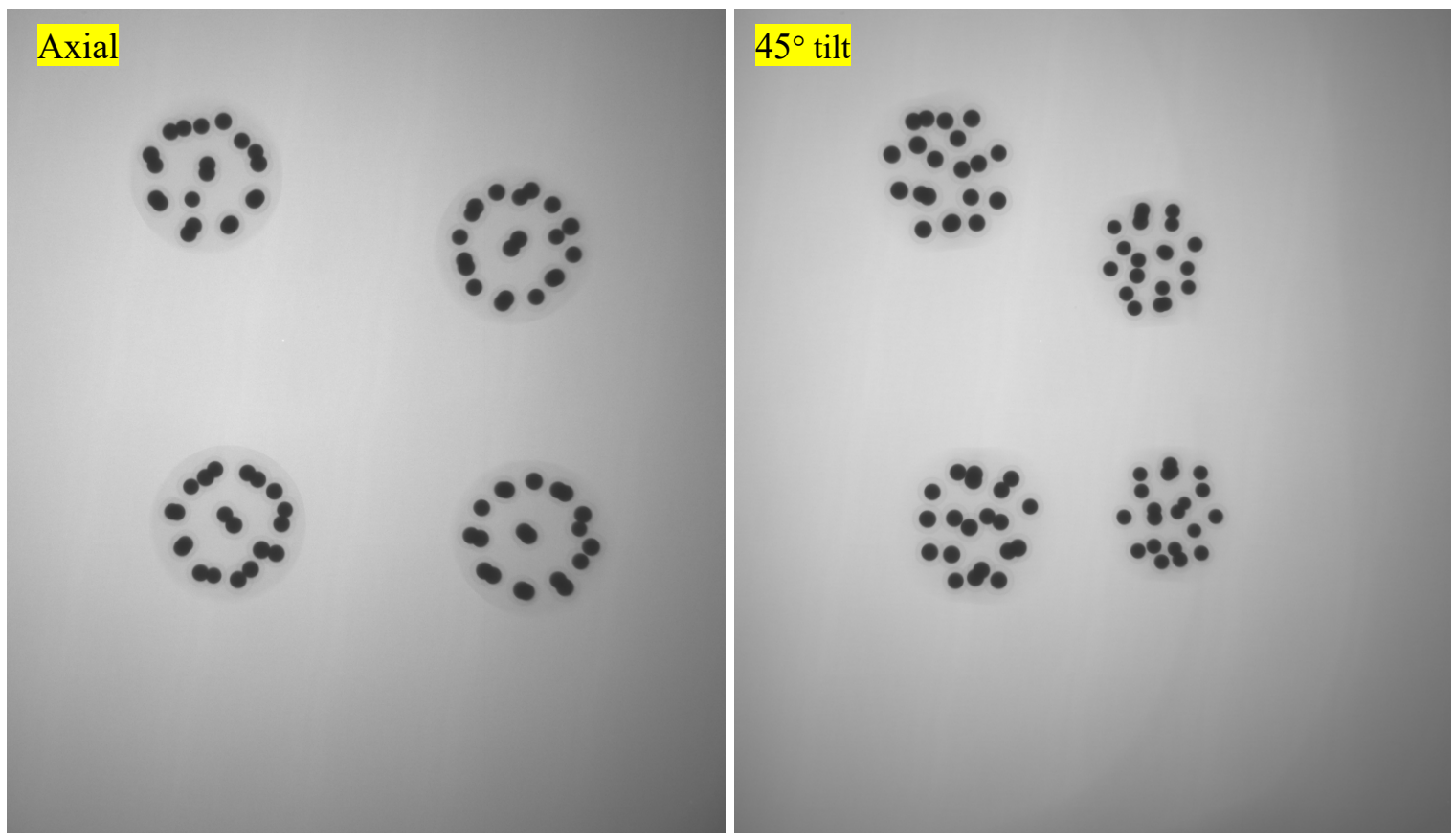

Figure 4. Example radiograph sets of LEU11-M05E-LEU11-M08E.

The radiography analysis provided two criteria to support compact down-selection in addition to density measurements discussed previously. If 20 particles were not clearly resolved in either of the two perspectives, then the compact was not considered for irradiation. This was the most common rejection criteria because particles were often observed to be overlapping. This rejection criterion was considered conservative. The second criterion was related to particle distribution. In select compacts in the LEU09M\#\#E series, a gap was observed in the center of the compacts as shown in Figure 3. This gap might not be critical; however, it represented an observed variation in particle distribution, and it was used to refine the compact variability in the planned test to ensure a more uniform set of compacts for irradiation.

The radiography confirmed 20 particles were present in all compacts selected for irradiation. Table 7 lists the total uranium content per MiniFuel compact based on average particle uranium content and enrichment.

Table 7. Total uranium content of MiniFuel compacts and isotopic breakdown.

\begin{tabular}{|l|l|l|l|l|l|}
\hline & \multicolumn{5}{|l|}{ Weight per MiniFuel compact } \\
\hline & Assay & Total U (g) & ${ }^{234} \mathbf{U}(\mathbf{g})$ & ${ }^{235} \mathbf{U}(\mathrm{g})$ & ${ }^{238} \mathbf{U}(\mathrm{g})$ \\
\hline LEUCO & $0.710 \%$ & $7.61 \mathrm{E}-03$ & $3.93 \mathrm{E}-07$ & $5.41 \mathrm{E}-05$ & $7.57 \mathrm{E}-03$ \\
\hline LEUO $_{2}$ & $14.174 \%$ & $7.62 \mathrm{E}-03$ & $7.16 \mathrm{E}-06$ & $1.08 \mathrm{E}-03$ & $6.53 \mathrm{E}-03$ \\
\hline NUCO & $9.580 \%$ & $1.22 \mathrm{E}-02$ & $7.47 \mathrm{E}-06$ & $1.16 \mathrm{E}-03$ & $1.10 \mathrm{E}-02$ \\
\hline
\end{tabular}




\subsubsection{Particle Position Analysis}

Select compacts were subjected to 3D XCT analysis (Table 3-Table 6). The whole compact tomography was conducted to support downstream PIE, particularly through refinement of thermal models to more accurately assess the compact and particle temperatures. The inputs that can be provided from 3D tomography analysis, specifically the particle positions and kernel volumes, will be used for thermal analysis to better predict the temperature distributions experienced across individual compacts as the current inputs assume a regular distribution of particles within the compact volume.

Each targeted compact was imaged by using a Zeiss Versa 520 XCT instrument. A series of 1,600 radiographs was acquired for each compact distributed over $360^{\circ}$ of rotation with the x-ray source set to a maximum energy of $40 \mathrm{kV}$ with no filter to maximize contrast of the low- $\mathrm{Z}$ graphite of the compact matrix. As shown in Figure 5, these settings sufficed to identify the outer edges of the compact and the positions of each kernel. Silicon carbide layers around each particle were partially distinguishable with some issues arising from image artifacts caused by the near-total blocking of $\mathrm{x}$-rays by the high-Z kernels. These issues could be resolved by applying advanced model-based image reconstruction methods with beam-blocking corrections.

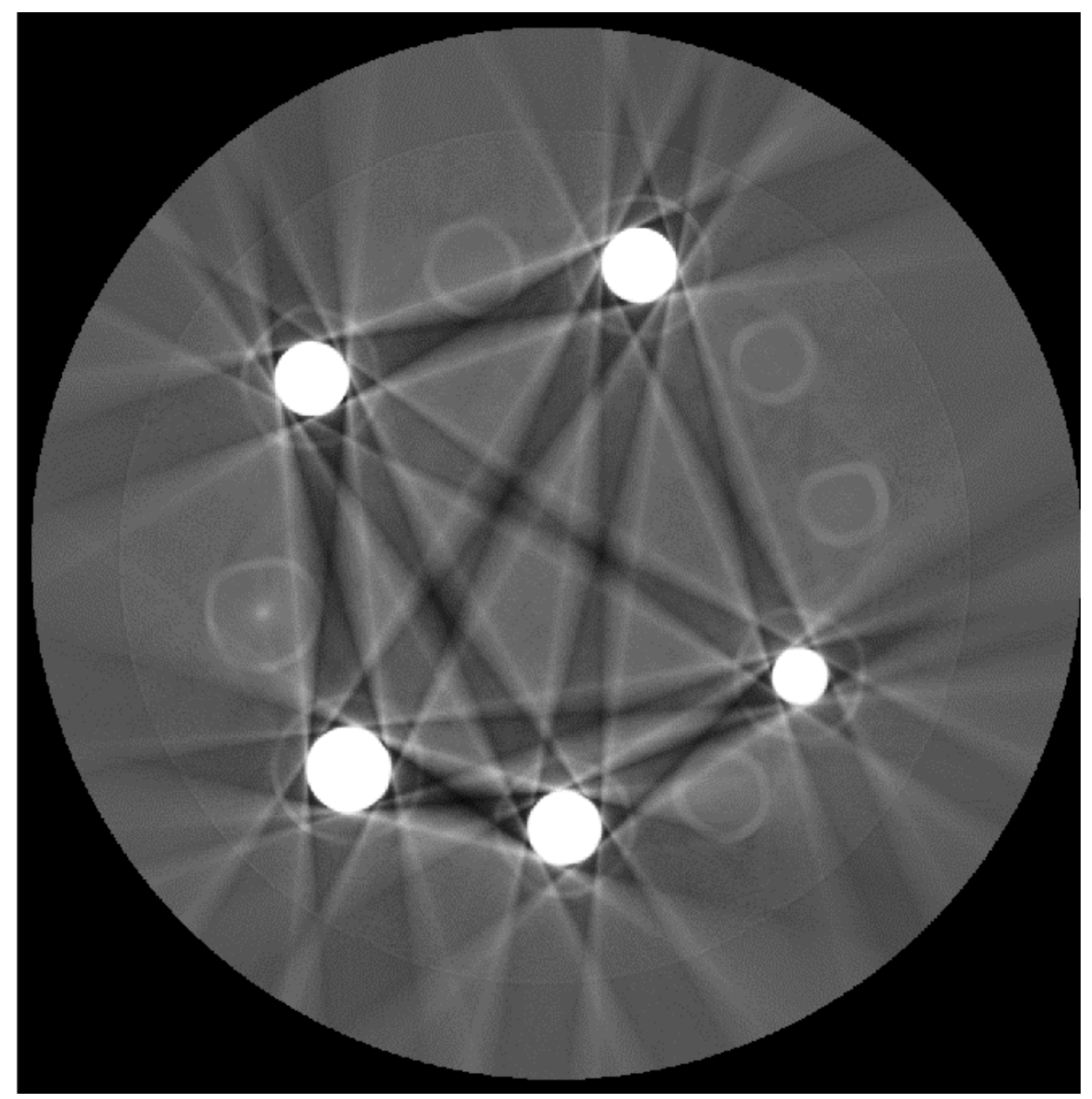

Figure 5. Cross-sectional tomogram from 3D tomograph of a compact showing kernels and $\mathrm{SiC}$ layers from several particles. 
A previously developed software for analyzing XCT images of particle fuel forms was used to analyze each compact (Helmreich et al. 2020b). This software determined the centroid positions of each kernel relative to the body of the compact, as well as the volume of each kernel. Centroid data were then used to determine secondary parameters for each kernel. A watershed segmentation of the compact around each kernel centroid was used to determine the compact volume occupied by each particle, which can be used as a measure of extremely localized packing fraction. The distance from the centroid of each particle to the compact surface (surface distance) was calculated based on the automatic segmentation of the compact boundaries. Finally, the identity and distance (centroid to centroid) to the three nearest neighbors for each particle were determined. An example of the data generated for a single compact is given in Table 8. All XCT generated data from analyzed compacts is presented in Appendix B.

Table 8. XCT data for particles in compact NUCO-M01F.

\begin{tabular}{|c|c|c|c|c|c|c|c|c|}
\hline \multicolumn{3}{|c|}{ Centroid $(\mu \mathrm{m})$} & \multicolumn{2}{|c|}{ Volume $\left(\mathrm{mm}^{3}\right)$} & \multirow{2}{*}{ Surface distance $(\mu \mathrm{m})$} & \multicolumn{3}{|c|}{ Nearest neighbor $(\mu \mathrm{m})$} \\
\hline$x$ & $y$ & $z$ & Kernel & Region & & 1st & 2nd & 3rd \\
\hline 237 & 1,420 & 506 & 0.0437 & 1.895 & 449 & 1,042 & 1,070 & 1,263 \\
\hline$-1,342$ & 265 & 555 & 0.0425 & 2.058 & 495 & 986 & 1,142 & 1,220 \\
\hline 34 & -277 & 557 & 0.0410 & 2.461 & 501 & 1,379 & 1,478 & 1,493 \\
\hline$-1,083$ & $-1,260$ & 680 & 0.0430 & 1.889 & 622 & 927 & 1,050 & 1,222 \\
\hline 1,298 & 759 & 683 & 0.0457 & 2.438 & 628 & 1,158 & 1,263 & 1,277 \\
\hline-788 & 1,324 & 797 & 0.0441 & 2.174 & 686 & 1,029 & 1,070 & 1,220 \\
\hline 1,429 & -810 & 811 & 0.0436 & 2.499 & 669 & 1,033 & 1,060 & 1,253 \\
\hline 7 & $-1,764$ & 909 & 0.0422 & 2.350 & 582 & 1,127 & 1,220 & 1,222 \\
\hline$-1,548$ & -555 & 1,062 & 0.0420 & 1.591 & 659 & 927 & 958 & 986 \\
\hline 1,694 & -30 & 1,433 & 0.0458 & 1.713 & 587 & 902 & 1,033 & 1,157 \\
\hline 581 & 1,537 & 1,482 & 0.0441 & 2.160 & 580 & 963 & 1,042 & 1,326 \\
\hline 873 & $-1,375$ & 1,516 & 0.0409 & 1.853 & 708 & 984 & 1,060 & 1,080 \\
\hline$-1,566$ & 419 & 1,664 & 0.0409 & 2.318 & 645 & 1,142 & 1,145 & 1,146 \\
\hline-819 & $-1,329$ & 1,695 & 0.0426 & 1.969 & 773 & 979 & 1,050 & 1,080 \\
\hline-794 & 1,394 & 1,823 & 0.0407 & 2.501 & 623 & 1,029 & 1,254 & 1,424 \\
\hline 91 & 38 & 1,898 & 0.0467 & 2.907 & 651 & 1,379 & 1,527 & 1,542 \\
\hline 1,268 & 1,032 & 1,930 & 0.0436 & 2.102 & 606 & 963 & 1,248 & 1,277 \\
\hline$-1,469$ & -669 & 2,011 & 0.0452 & 1.808 & 541 & 958 & 979 & 1,146 \\
\hline 1,451 & -639 & 2,052 & 0.0423 & 1.899 & 501 & 902 & 1,079 & 1,253 \\
\hline 133 & $-1,611$ & 2,119 & 0.0394 & 1.799 & 432 & 984 & 1,079 & 1,227 \\
\hline
\end{tabular}

These data can be visualized for a subset of particles or for the full compact, as shown in Figure 6 and Figure 7. The particle configuration in this compact is typical for all compacts analyzed with three layers of particles at approximately the same height in the compact and $\sim 6-8$ particles within each layer. The consistency of the particle distribution in the XCT analyzed MiniFuel compacts reflects the decision to not select compacts which had no central particles (Figure 3). 


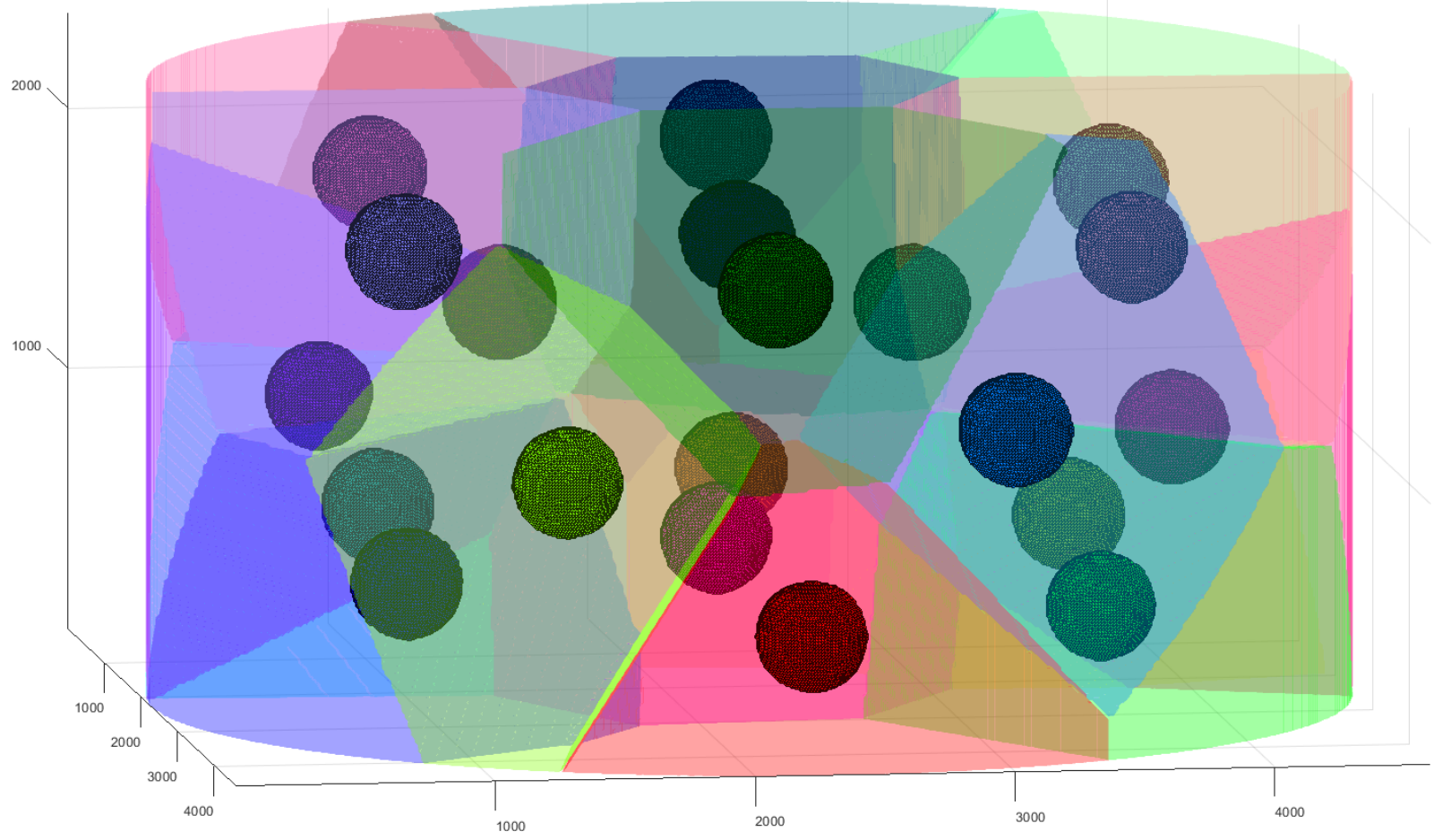

Figure 6. Side view of a 3D representation of an XCT image for a compact showing each particle and its associated region volume within the compact in a unique color.

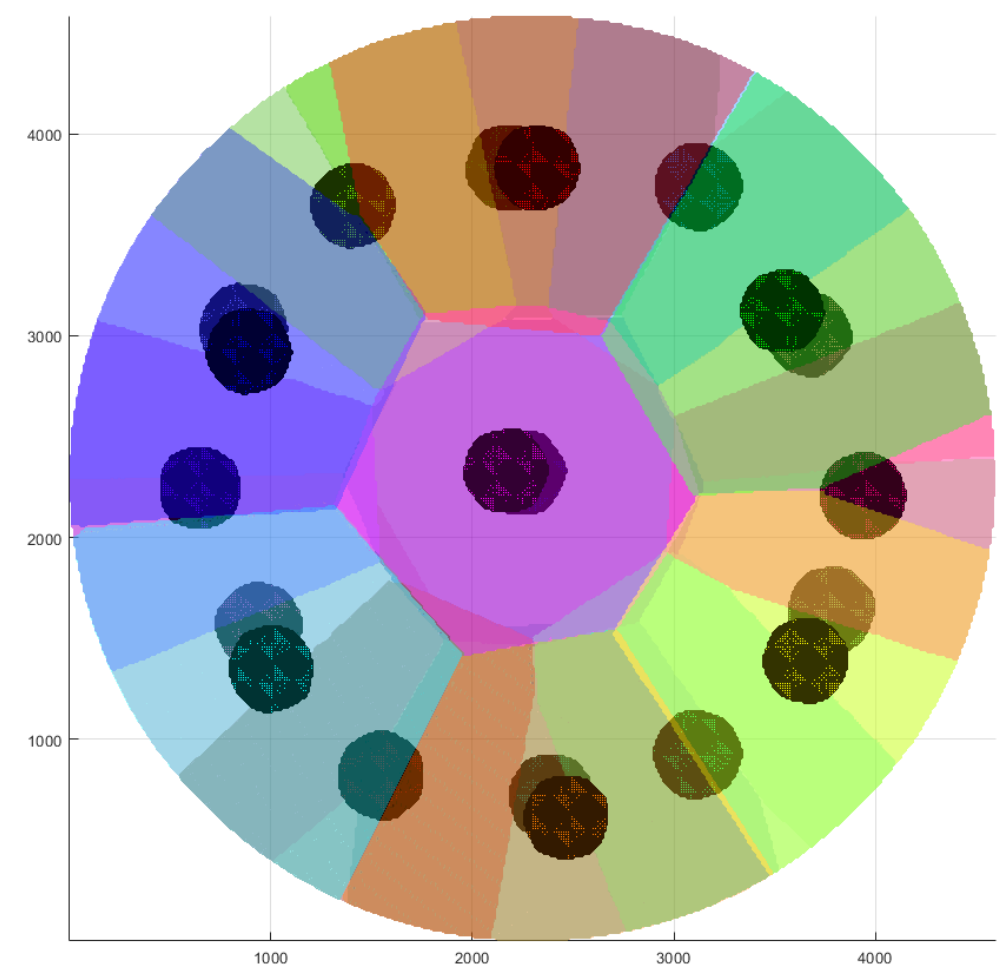

Figure 7. Top-down view of a 3D representation of an XCT image for a compact showing each particle and its associated region volume within the compact in a unique color. 


\subsubsection{Particle Failure Fraction Analysis}

One primary metric for TRISO fuel performance is particle failure fraction. Modern TRISO particles have shown failure fractions better than $\leq 5.1 \mathrm{E}-5$ at $95 \%$ confidence (Demkowicz et al. 2019). The planned irradiation intends to explore margin irradiation conditions and potentially push the operating envelope for fuel performance. Determining the as-fabricated defect fraction is important for interpreting failures observed in-pile relative to failure that might have been present from as-fabricated defects. The asfabricated defect fraction for AGR-2 particles for $\mathrm{UCO}$ and $\mathrm{UO}_{2}$ kernel variants measured multiple defect types, including uranium contamination fraction, defective $\mathrm{SiC}$ coating fraction, defective IPyC coating fraction, and defective OPyC coating fraction (Hunn et al. 2010a, Hunn et al. 2010b). The uranium contamination fraction and defective $\mathrm{SiC}$ coating fraction were equivalent to the defect fraction measured in this work because the values reflect the number of exposed kernel defects pre- and post-burn. The maximum reported failure fraction for the two AGR-2 fuel variants after compacting was $\leq 3.2 \mathrm{E}-5$ at a 95\% confidence level (Hunn et al. 2010a, Hunn et al. 2010b). The failure rate at a 95\% confidence level was determined from a burn-back analysis of the MiniFuel compacts. MiniFuel compacts were subjected to a burn step similar to the burn step in the deconsolidation leach-burn-leach process (Hunn et al. 2013). MiniFuel compact clutches that contained no more than 120 particles each were subjected to a burn under forced air in a muffle furnace at $750^{\circ} \mathrm{C}$ for $48 \mathrm{~h}$. The particle defect fraction was then determined by performing x-ray radiography analysis by using the same Zeiss Versa $520 \mathrm{XCT}$ instrument. The radiographs were analyzed to look for defective particles. Defective particles with exposed kernel defects are indicated by fractured $\mathrm{SiC}$, oxidized (i.e., swollen) kernels, and/or oxidized (i.e., missing) IPyC and buffer layers. Figure 8 provides an example of an exposed kernel defect after the burn step (Gerczak et al. 2016 ) in which a particle with a compromised SiC layer led to the burning out of the internal IPyC and buffer layers and the kernel swelling after exposure to forced air at $750^{\circ} \mathrm{C}$.

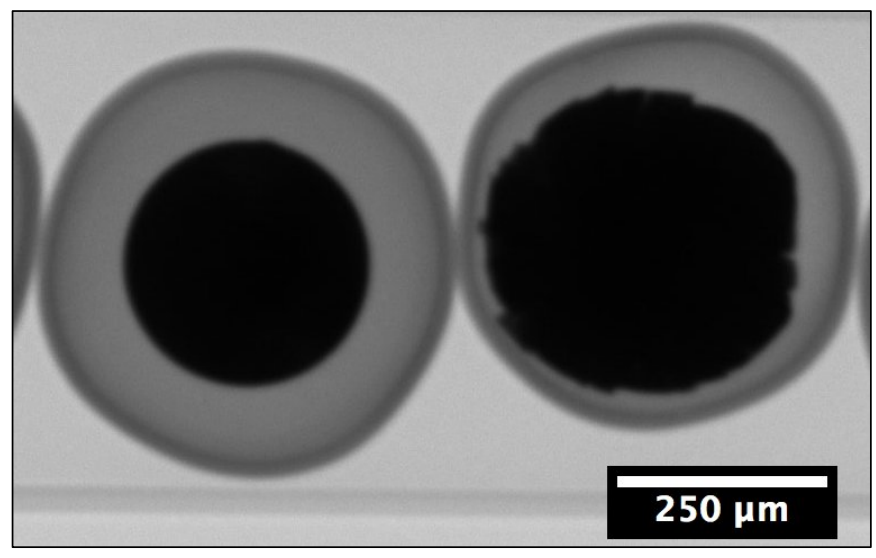

Figure 8. Example radiograph of an intact particle (left) and oxidized kernel and missing buffer/IPyC particle (right) after exposure to a burn stage (Gerczak et al. 2016).

Figure 9 shows an example radiograph of a burn test particle clutch. Each radiograph was analyzed for irregular or defect particles. A total of 1,360 particles were analyzed from the compacts selected for burn testing listed in Table 3-Table 6. No defective or failed particles were observed. The analysis reflected an as-fabricated defect fraction of $\leq 2.2 \mathrm{E}-3$ at $95 \%$ confidence. This number serves as a baseline for establishing in-pile failures during irradiation. All radiographs used for defect analysis are shown in Appendix C. 


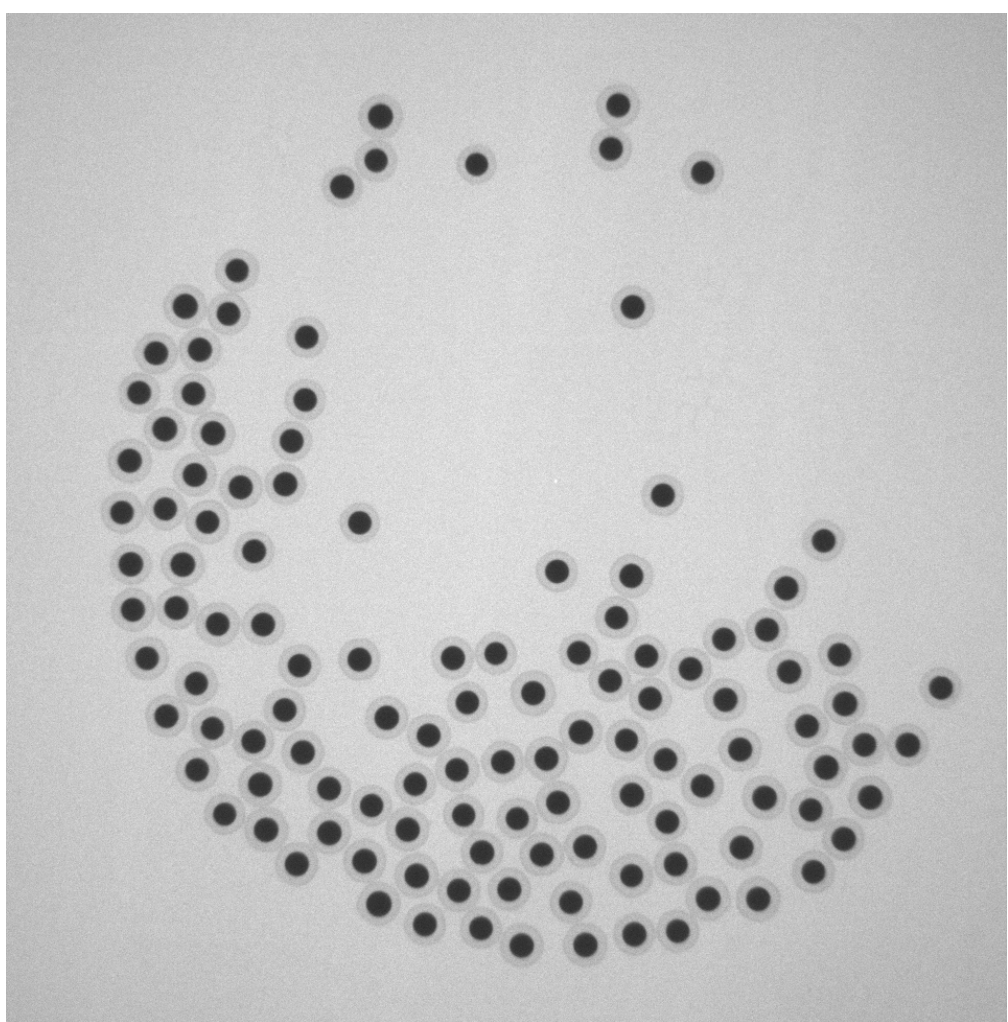

Figure 9. Example of radiography analysis of a 120 particle clutch after $750^{\circ} \mathrm{C}$ for $48 \mathrm{~h}$ under forced air from LEU09-M\#\#E.

\subsection{MATRIX IMPURITY ANALYSIS}

Impurity content in the matrix was measured to support safety analysis and determine whether excess impurities were present. Excess impurities content is needed for safety analysis and is also needed to understand the potential for deleterious interactions with the TRISO particles in the MiniFuel compacts. Matrix samples were prepared and sent to Eurofins EAG Materials Science LLC for impurity analysis by glow discharge mass spectroscopy. The data were reported in concentration. The impurity analysis results are listed in Table 9 and are compared with specified limits, measured impurity, and contamination data from AGR-2 compacts (Collin 2014) data. The AGR-2 data were converted from the micrograms of each impurity outside the $\mathrm{SiC} /$ compact to parts-per-million weight by dividing by the average matrix material weight per compact. This is an estimate because it does not account for the OPyC layer weight. The data in Table 9 confirms that the impurity concentration was below the specified values and were on the order of or better than the measured impurity concentrations in the AGR-2 compacts. 
Table 9. Select impurity data for matrix material from MiniFuel compacts.

\begin{tabular}{|c|c|c|c|c|}
\hline & \multicolumn{4}{|c|}{ All values in parts-per-million by weight } \\
\hline Impurity & $\begin{array}{c}\text { Specific range } \\
\text { for AGR-2* }\end{array}$ & $\begin{array}{c}\text { Values for AGR-2 } \\
\text { UCO compacts* }\end{array}$ & $\begin{array}{c}\text { Values for AGR-2 } \\
\text { UO }_{2} \text { compacts* }\end{array}$ & This work \\
\hline Iron & $\leq 8.3$ & 1.34 & 0.71 & 0.46 \\
\hline Chromium & $\leq 16.6$ & 0.20 & 0.12 & $<1$ \\
\hline Manganese & $\leq 16.6$ & 0.05 & 0.03 & $<0.05$ \\
\hline Cobalt & $\leq 16.6$ & 0.37 & 0.03 & $<0.05$ \\
\hline Nickel & $\leq 16.6$ & 0.32 & 0.15 & 0.17 \\
\hline Calcium & $\leq 16.6$ & 13.04 & 9.14 & 2.9 \\
\hline Aluminum & $\leq 16.6$ & 9.81 & 11.10 & 0.55 \\
\hline Titanium & - & 0.93 & 0.86 & 0.89 \\
\hline Vanadium & - & 5.67 & 4.01 & 1.4 \\
\hline Uranium** & - & 1.59 & 1.57 & $<0.05$ \\
\hline Molybdenum & - & - & - & $<0.1$ \\
\hline
\end{tabular}

*Source data from Collin (2014), values converted to parts-per-million weight by dividing by the average matrix weight per compact.

**Contamination fraction reported as $\mathrm{g} \mathrm{U} / \mathrm{g} \mathrm{U}$ in compact without exposed kernels.

\section{SUMMARY}

MiniFuel compacts were subjected to a series of preirradiation characterization techniques to provide relevant data for irradiation capsule design and HFIR safety analysis. The data were necessary for providing inputs to refine thermal models and HFIR safety calculations. The analysis confirmed that all compacts selected for archive or irradiation contained the necessary 20 particles. The measured densities were within the targeted ranges associated with the AGR-1 irradiation experiment. The density measurements allowed outlier compacts to be removed and compacts with a reasonable variance in density to be selected. Other down-selection criteria, such as having no particles in the compact center, were identified to support a more uniform comparison across compacts included in the planned irradiation. The XCT analysis provided unique insight into the kernel volume and particle positions within the individual compacts. These data will be critical for refining thermal modeling to better support temperature analysis and the interpretation of PIE results. Lastly, the as-fabricated defect analysis indicated that no particles failed during compact fabrication and provided a measure of the as-fabricated defects fraction at a $95 \%$ confidence level. The defect fraction will be critical when interpreting potential in-pile failures and the influence of irradiation conditions on TRISO particle performance. 


\section{REFERENCES}

Brown, N.R., Betzler, B.R., Carbajo, J.J., Wysocki, A.J., Greenwood, M.S., Gentry, C., Qualls, A.L., 2017. "Preconceptual Design of a Fluoride High Temperature Salt-cooled Engineering Demonstration Reactor: Core Design and Safety Analysis," Ann. Nucl. Energy. 103, 49-59.

Bullock, R.E., Kaae, J.L., 1983. "Performance of Coated $\mathrm{UO}_{2}$ Particles Gettered with ZrC," J. Nucl. Mater., 115, 69-83.

BWXT, 2008a. Industrial Fuel Fabrication and Development Lot G73AA-10-69308. Data Certification Package.

BWXT, 2008b. Industrial Fuel Fabrication and Development Lot G73I-14-69307. Data Certification Package.

BWXT, 2008c. Industrial Fuel Fabrication and Development Lot G73J-14-93071A, G73J-14-93073A, G73J-14-93074A. Data Certification Package.

BWXT, 2009. Industrial Fuel Fabrication and Development Lot G73H-10-93085B. Data Certification Package.

Collin, B., 2014. AGR-2 Irradiation Test Final As-Run Report. INL/EXT-14-32277 Rev. 1. Idaho National Laboratory, Idaho Falls, Idaho. 2014.

Gallagher, R. C., Wallen, Z., Petrie, C. M., Gerczak., T. J., Le Coq, A., Smith, K., Harp, J., Linton, K., Collin, B., Latta, R., 2020. Analysis and Design of High-Power TRISO Fuel Compact Irradiation in HFIR. ORNL/TM-2020/1658, Oak Ridge National Laboratory, Oak Ridge, Tennessee.

Gerczak, T.J., Baldwin, C.A., Helmreich, G.W., Hunn, J.D., Montgomery, F.C., 2016. Preparation of Simulated LBL Defects for Round Robin Experiments. ORNL/TM-2015/722. Rev. 2.

Gerczak, T. J., Contescu, C., Lee, Y. J., Mee, R., Schumacher, A. T., Stempien, J., and Trammell, M. 2019. Oxidation of Matrix Material in Helium with Varied Moisture Content. ORNL/TM-2019/1341. Rev. 0.

Gerczak, T.J., Kercher, A., Schumacher, A.T., Helmreich, G.W., Trammell, M., 2020. Fabrication of MiniFuel Compacts for High-Power Irradiation Testing of TRISO Fuel. ORNL/TM-2020/1778. Rev. 0.

Helmreich, G.W., Hunn, J.D., McMurray, J.W., Brown, D.R., 2020a. "Enhanced Method for Analysis of Individual UCO Kernel Phase Fractions,” Nucl. Eng. Des., 363, 110625.

Helmreich, G.W., Hunn, J.D., Brown, D.R., Blamer, B.J., 2020b. "New Method for Analysis of X-ray Computed Tomography Scans of TRISO Fuel Forms," Nucl. Eng. Des., 357, 110418.

Homan, F.J., Lindemer, T.B., Long Jr., E.L., Tiegs, T.N., Beatty, R.L., 1977. "Stoichiometric Effects on Performance of High Temperature Gas Cooled Reactor Fuels from the U C O System," Nucl. Tech. $35,428-441$.

Hunn, J.D., 2008. Data Compilation for AGR-2 B\&W UCO Coated Particle Batch G73J-14-93073A. ORNL/TM-2008/134. Rev. 1.

Hunn, J.D., 2010. Data Compilation for AGR-2 B\&W UO Coated Particle Batch G73H-10-93085B. ORNL/TM-2010/255. Rev. 1.

Hunn, J. D., Montgomery, F. C., and Pappano, P. J., 2010a. Data Compilation for AGR-2 B\&W UCO Compact Lot LEU09-OP-Z. ORNL/TM-2010/017. Rev. 1. 
Hunn, J. D., Montgomery, F. C., and Pappano, P. J., 2010b. Data Compilation for AGR-2 B\&WUO Compact Lot LEU11-OP-Z. ORNL/TM-2010/055. Rev. 1.

Hunn, J. D., Savage, T. W., Silva, G. W. C., 2010c. AGR-2 Fuel Compact Pre-Irradiation Characterization Summary Report. ORNL/TM-2010/226. Rev. 1.

Hunn, J. D., Trammell, M. P., and Montgomery, F. C., 2011. Data Compilation for AGR-3/4 Matrix Ring Blank Lot RDKRS. ORNL/TM-2011/127. Rev. 1.

Hunn, J .D., Savage, T. W., Silva, C. M., 2012. AGR-1 Fuel Compact Pre-Irradiation Characterization Summary Report. ORNL/TM-2012/295. Rev. 0.

Hunn, J. D., Morris, R. N., Baldwin, C. A., Montgomery, F. C., Silva, G. W. C., and Gerczak, T. J., 2013. AGR-1 Irradiated Compact 4-4-2 PIE Report: Evaluation of As-Irradiated Fuel Performance with Leach Burn Leach , IMGA , Materialography, and X-ray Tomography. ORNL/TM-2013/236, Oak Ridge National Laboratory, Oak Ridge, Tennessee.

Hunn, J. D., Lowden, R. A., Miller., J. H., Jolly., B. C., Trammell, M. P., Kercher, A. K., Montgomery., F. C., Silva., C. M., 2014. "Fabrication and Characterization of Driver-Fuel Particles, Designed-toFail Fuel Particles, and Fuel Compacts for the US AGR-3/4 Irradiation Test." Nucl. Eng. Des. 271: 123-130.

Latta, R., Collin, B., Hackett, M., Brown, N. R., Hunn, J. D., Petrie, C. M., Gerczak., T. J., and Helmreich., G. W., 2019. "High Power Irradiation Testing of TRISO Particles in Miniature Fuel Specimens in HFIR," ANS Transactions, 121, 1: 641-643.

Maki, J.T., Petti, D.A., Knudson, Miller, G.K., 2007. "The Challenge Associated with High Burnup, High Temperature and Accelerated Irradiation for TRISO Coated Particle Fuel," J. Nucl. Mater., 371, 270 280.

McMurray, J.W., Lindemer, T.B., Brown, N.R., Reif, T.J., Morris, R.N., 2017. "Determining the Minimum Required Uranium Carbide Content for HTGR UCO Fuel Kernels," Ann. Nucl. Energy., 104, 237-424.

Pappano, P. J., Burchell, T. D., Hunn, J. D., and Trammell, M. P., 2008. “A Novel Approach to Fabricating Fuel Compacts for the Next Generation Nuclear Plant (NGNP).” J. Nucl. Mater. 381, 12: $25-38$.

Pappano, P. J. and Hunn, J. D., 2008. "Update on Overcoating and Compacting Activities for Coated Particles with $425 \mu \mathrm{m}$ Kernels" Proceedings of the 4th International Topical Meeting on High Temperature Reactor Technology. HTR2008 September 28-October 1, 2008, Washington, DC.

Petrie, C. M., Burns, J. R., Raftery, A. M., Nelson, A.T ., and Terrani, K. A., 2019. "Separate Effects Irradiation Testing of Miniature Fuel Specimens," J. Nucl. Mater. 526, 151783.

Petti, D., Maki, J., Hunn, J., Pappano, P., Barnes, C., Saurwein, J., Nagley, S., Kendall, J., and Hobbins, R., 2010. "The DOE Advanced Gas Reactor Fuel Development and Qualification Program," JOMUS. 61: 62-66.

Seibert R.L., Terrani, K.A., Kiggans, J.O., McMurray, J.W., Jolly, B.C., Petrie, C.M., and Nelson, A.T., 2019. Fabrication and Irradiation Test Plan for Fully Ceramic Microencapsulated Fuels. ORNL/TM-2019/1088, Oak Ridge National Laboratory, Oak Ridge, Tennessee.

Terrani, K.A., Snead, L.L., Gehin, J.C., 2012. "Microencapsulated fuel technology for commercial light water and advanced reactor application," J. Nucl. Mater., 427. 209-224. 



\section{APPENDIX A.}

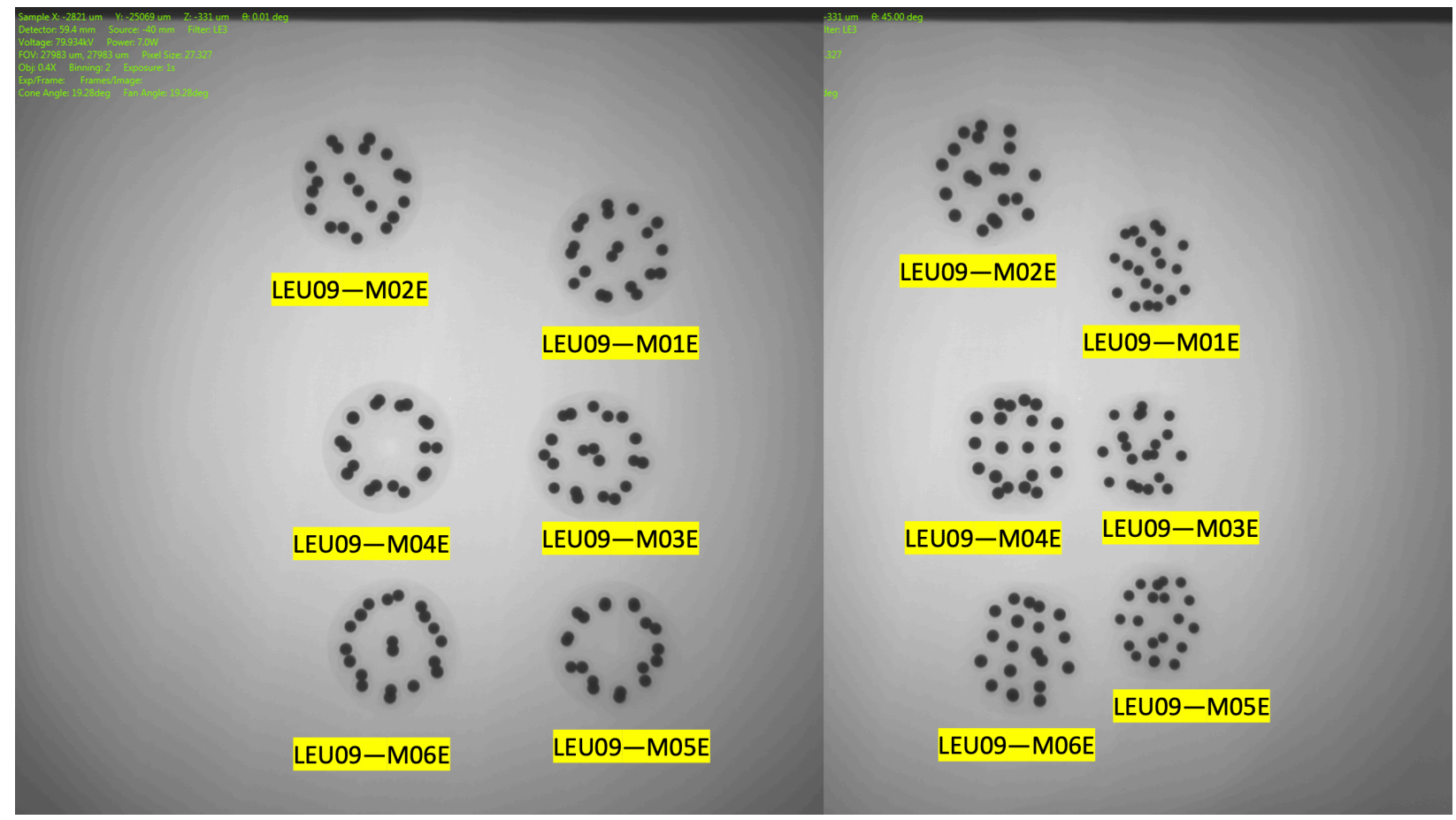

Figure A-1. Radiography sets of LEU09-M01E-LEU09-M06E; axial image orientation (left) and $45^{\circ}$ tilt from the axial direction (right).

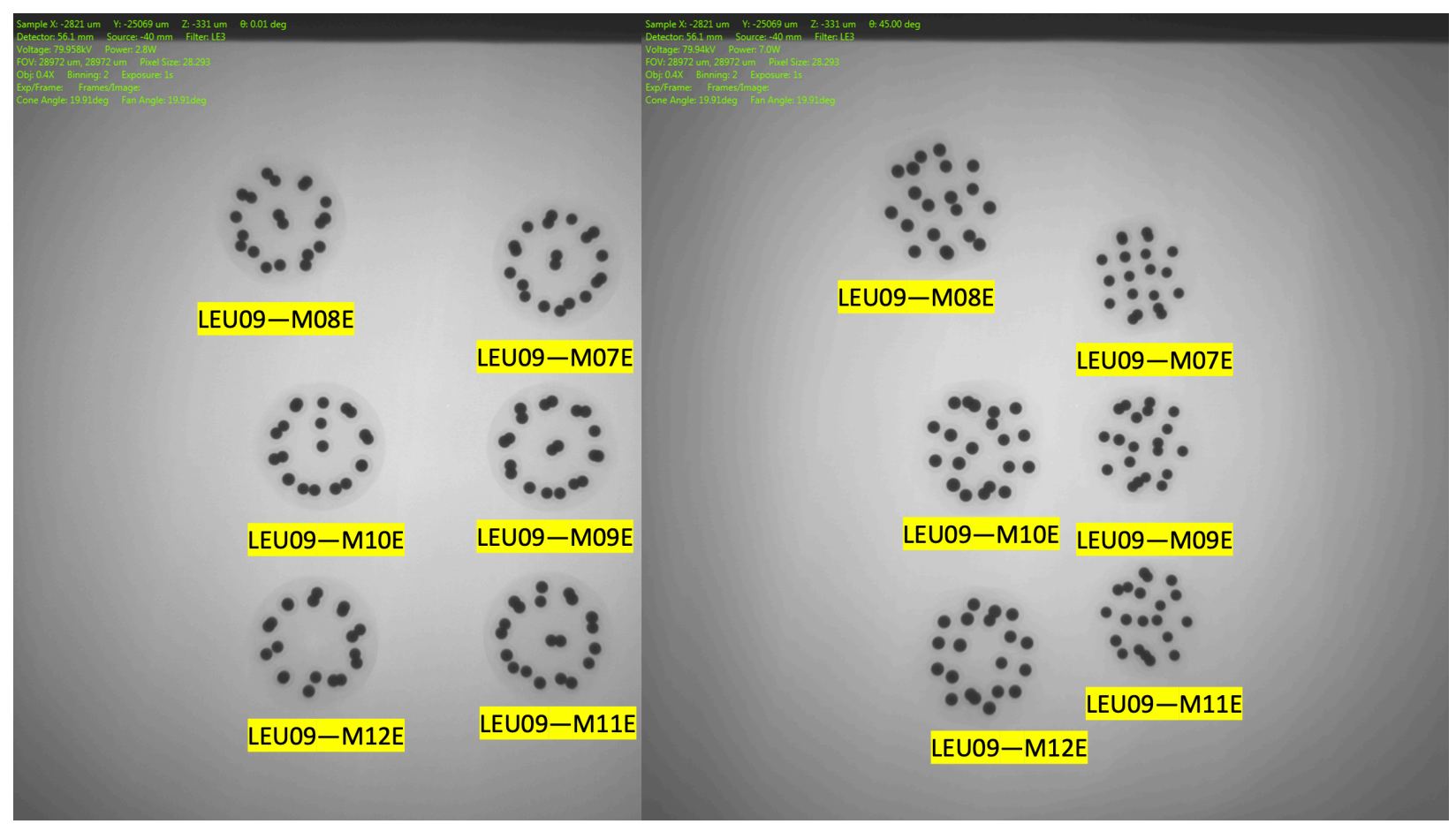

Figure A-2. Radiography sets of LEU09-M07E-LEU09-M12E; axial image orientation (left) and $45^{\circ}$ tilt from the axial direction (right). 


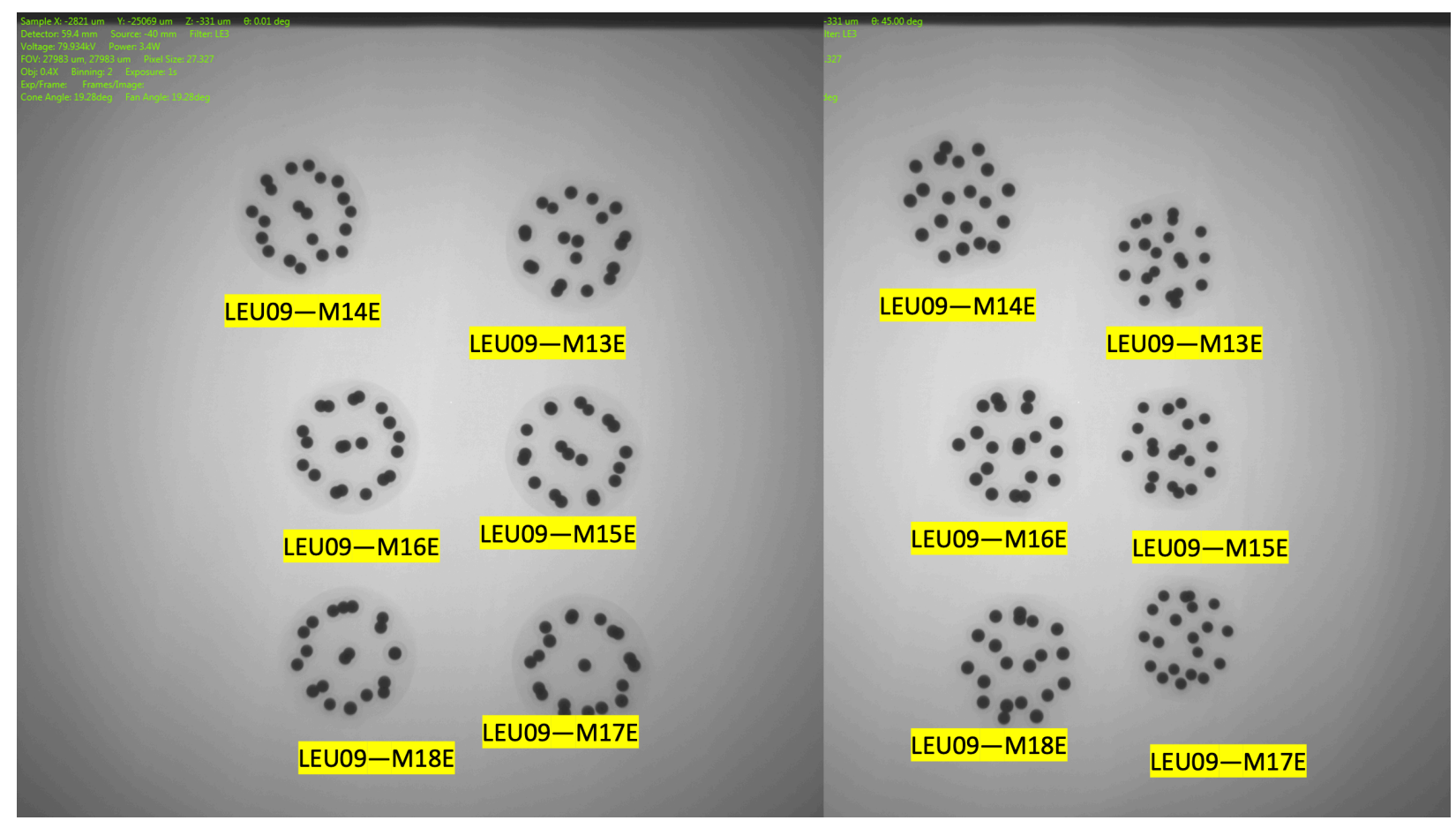

Figure A-3. Radiography sets of LEU09-M13E-LEU09-M18E; axial image orientation (left) and $45^{\circ}$ tilt from the axial direction (right).

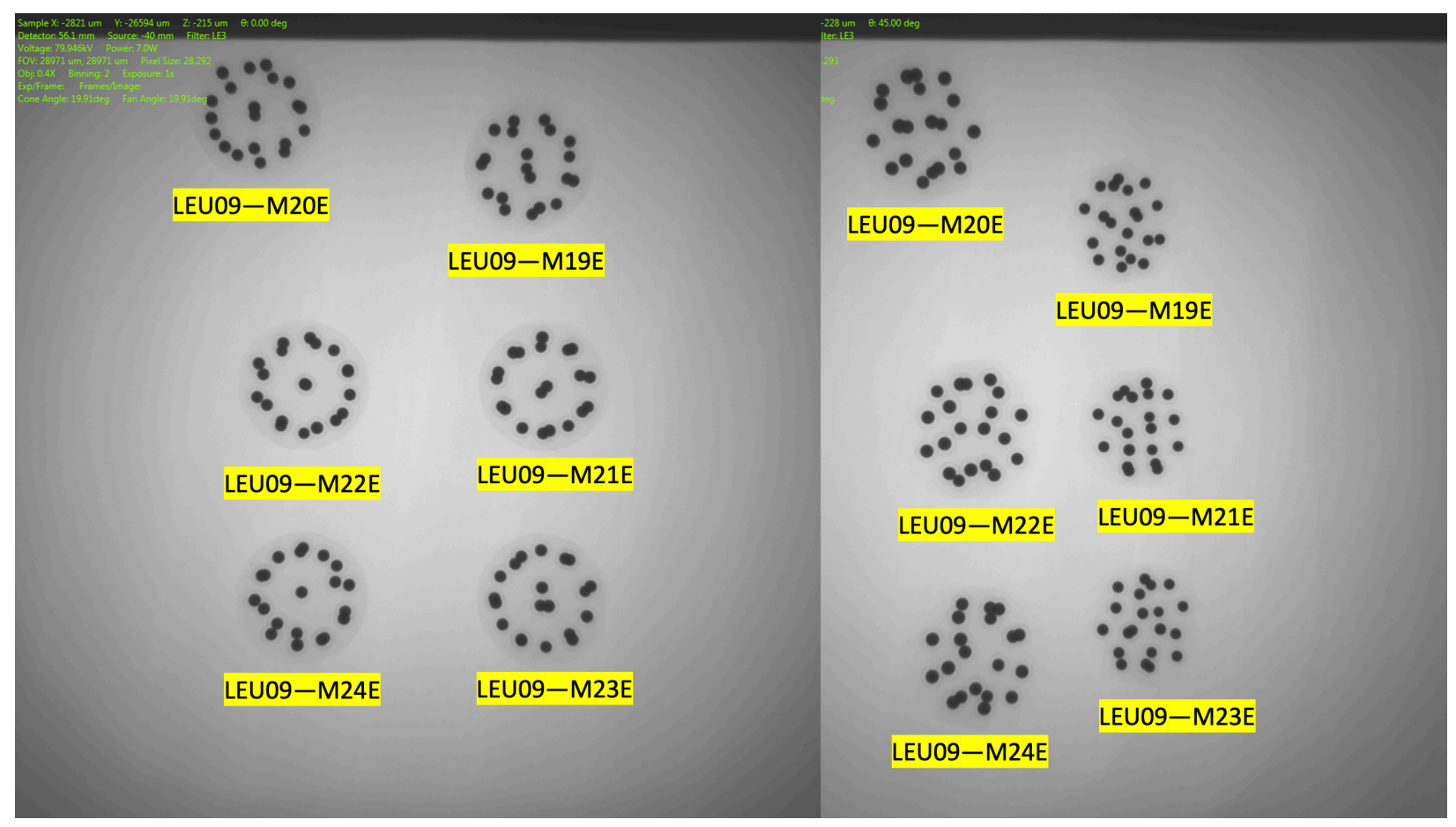

Figure A-4. Radiography sets of LEU09-M19E-LEU09-M24E; axial image orientation (left) and $45^{\circ}$ tilt from the axial direction (right). 


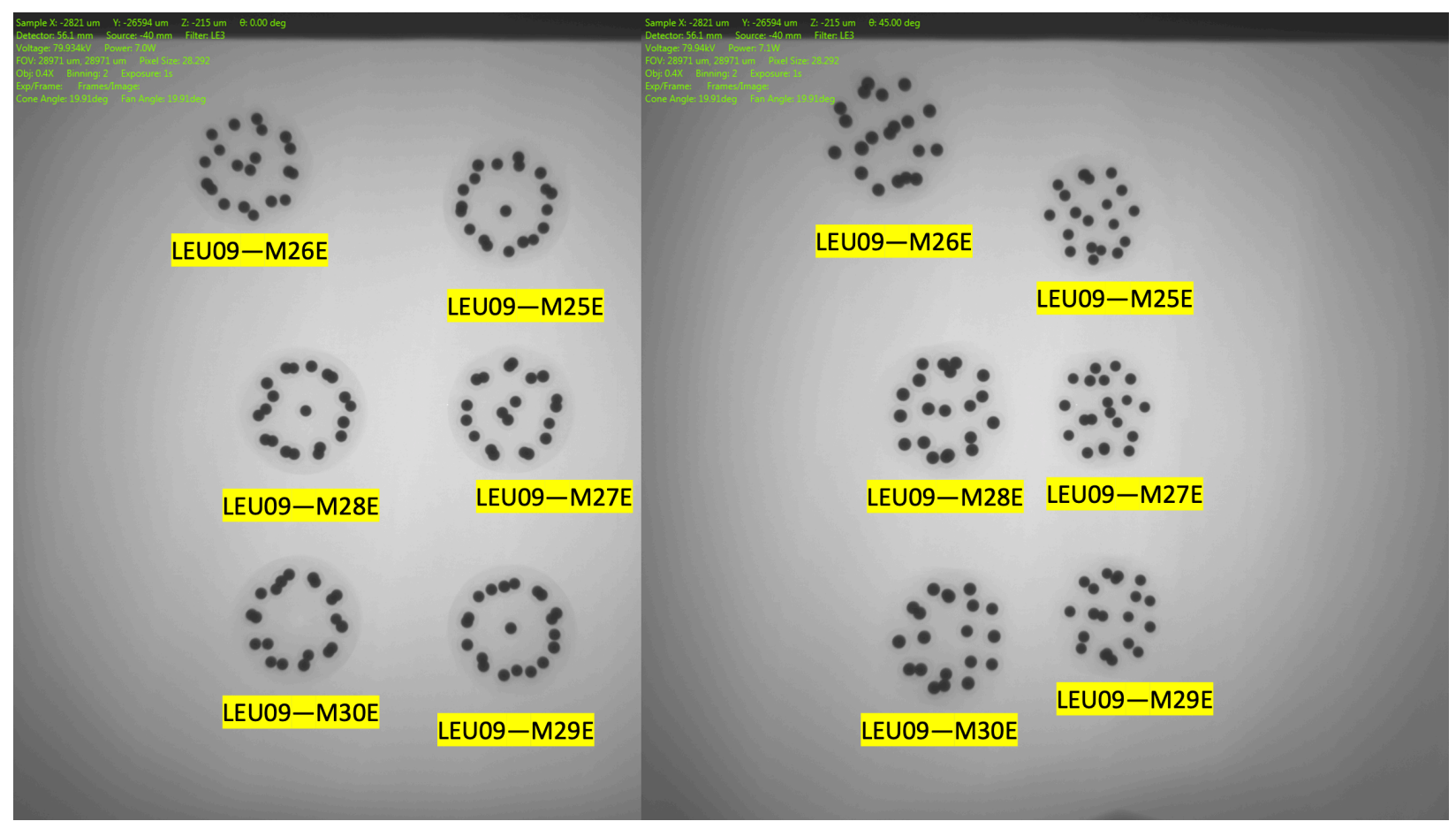

Figure A-5. Radiography sets of LEU09-M25E-LEU09-M30E; axial image orientation (left) and $45^{\circ}$ tilt from the axial direction (right).

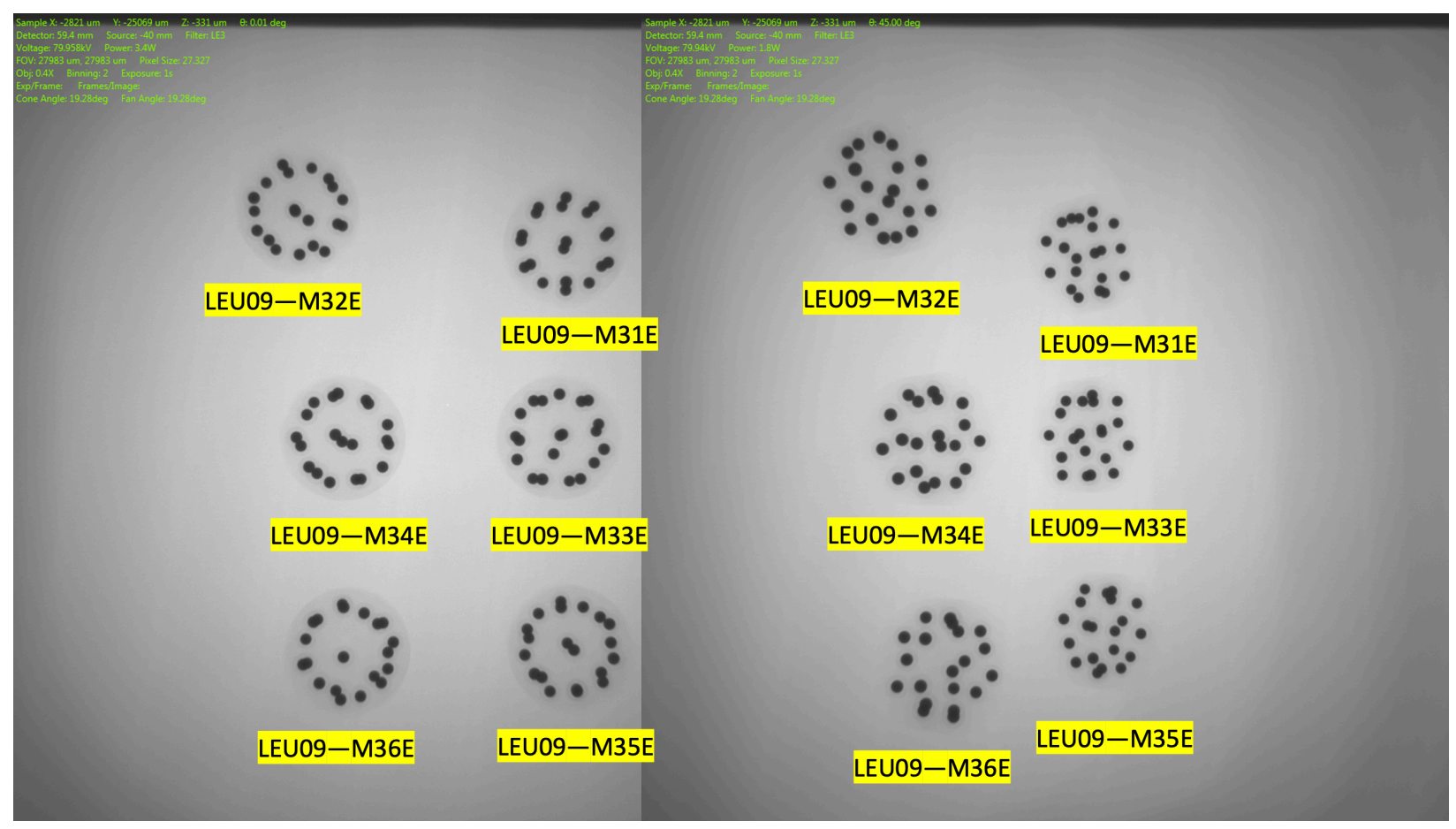

Figure A-6. Radiography sets of LEU09-M31E-LEU09-M36E; axial image orientation (left) and $45^{\circ}$ tilt from the axial direction (right). 


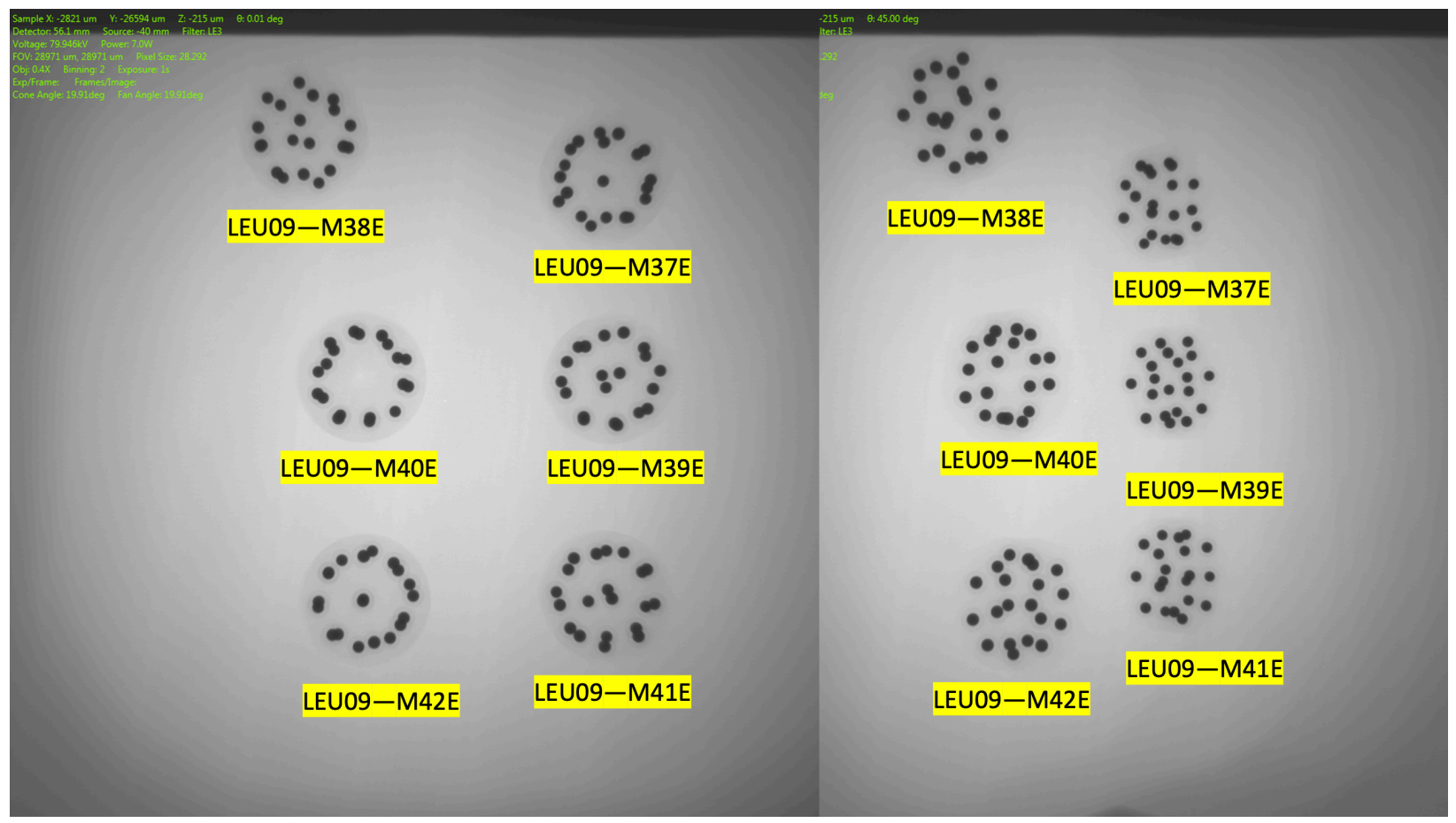

Figure A-7. Radiography sets of LEU09-M37E-LEU09-M42E; axial image orientation (left) and $45^{\circ}$ tilt from the axial direction (right).

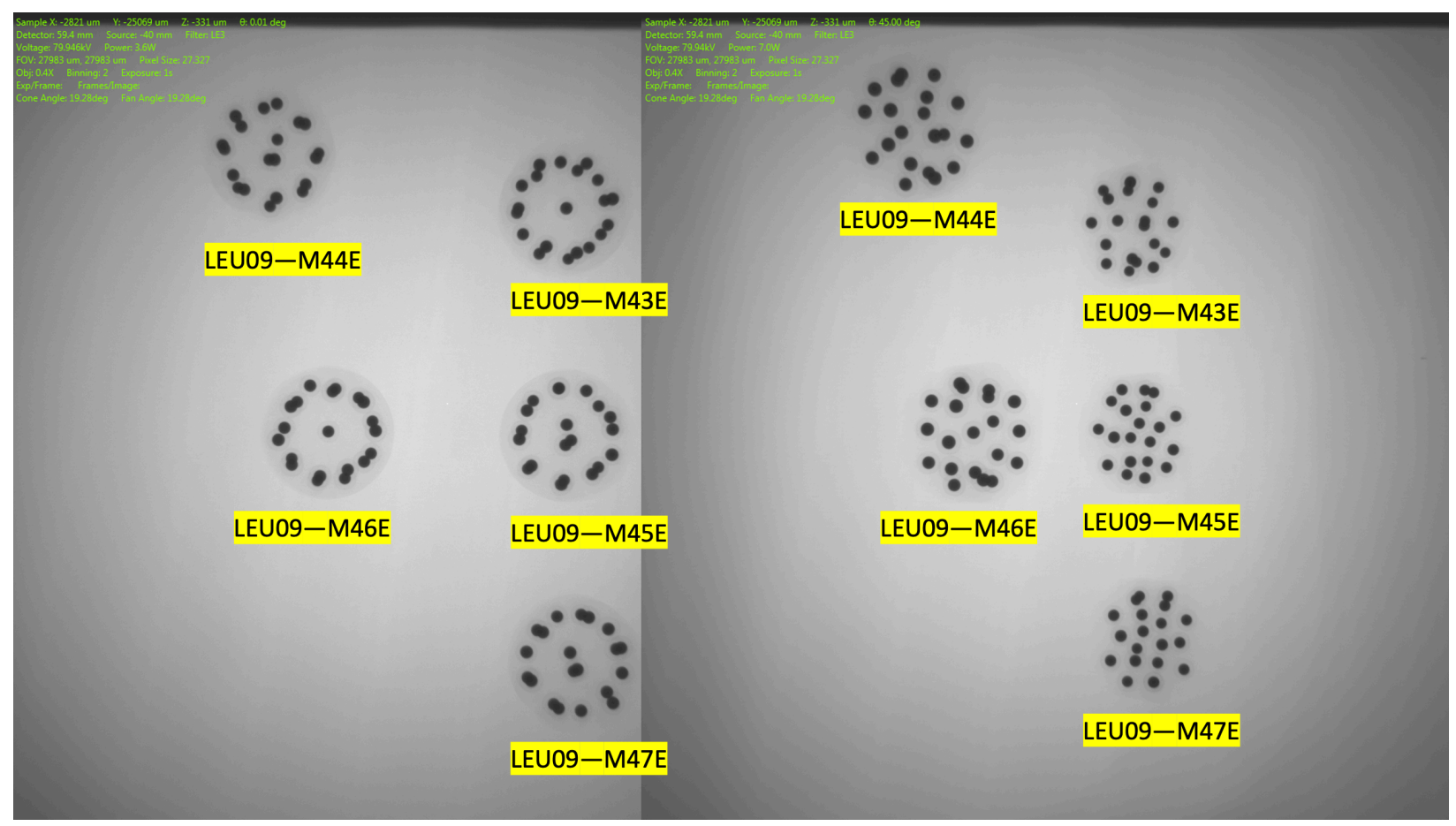

Figure A-8. Radiography sets of LEU09-M43E-LEU09-M47E; axial image orientation (left) and $45^{\circ}$ tilt from the axial direction (right). 


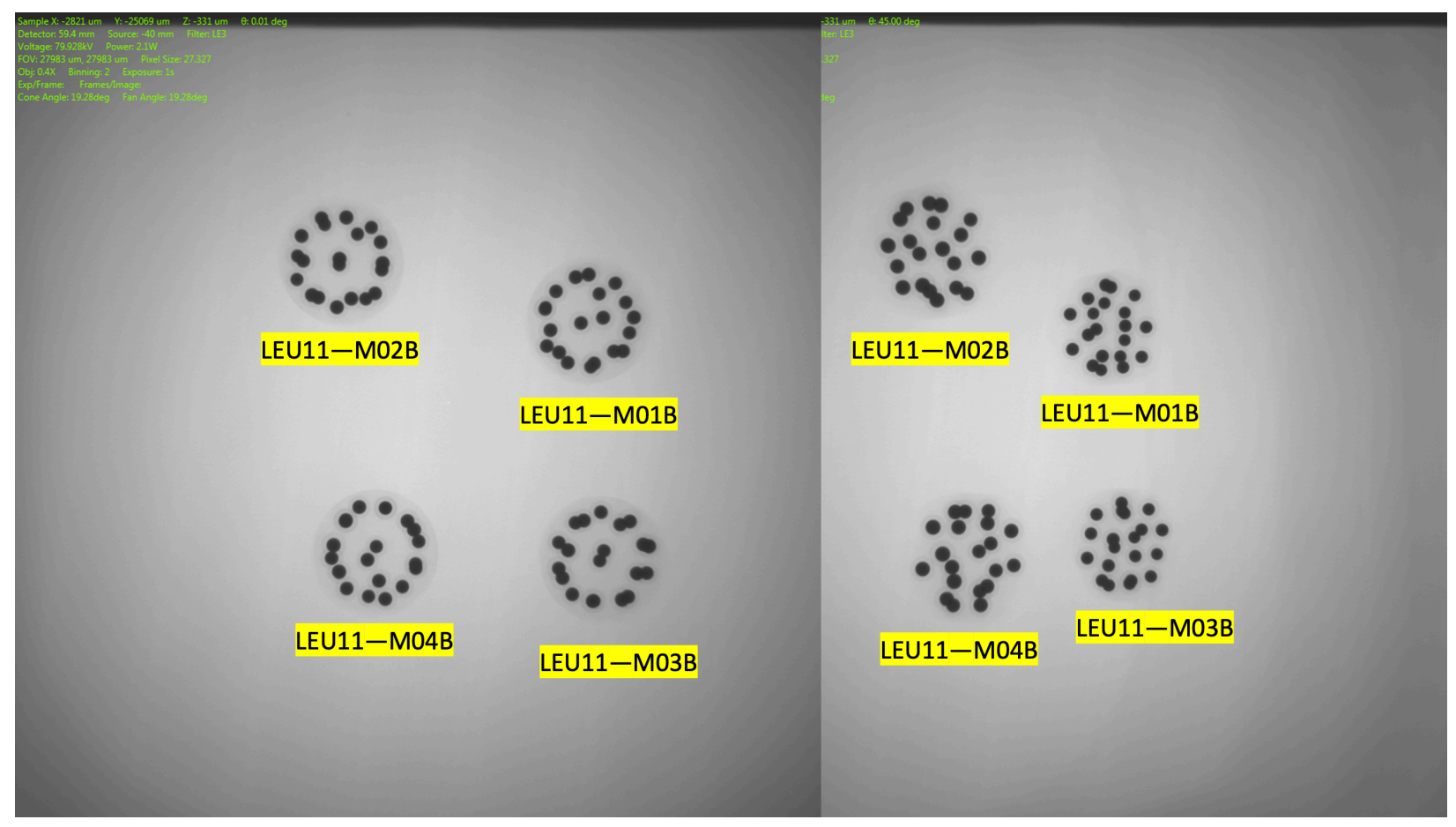

Figure A-9. Radiography sets of LEU11-M01B-LEU11-M04B; axial image orientation (left) and $45^{\circ}$ tilt from the axial direction (right).

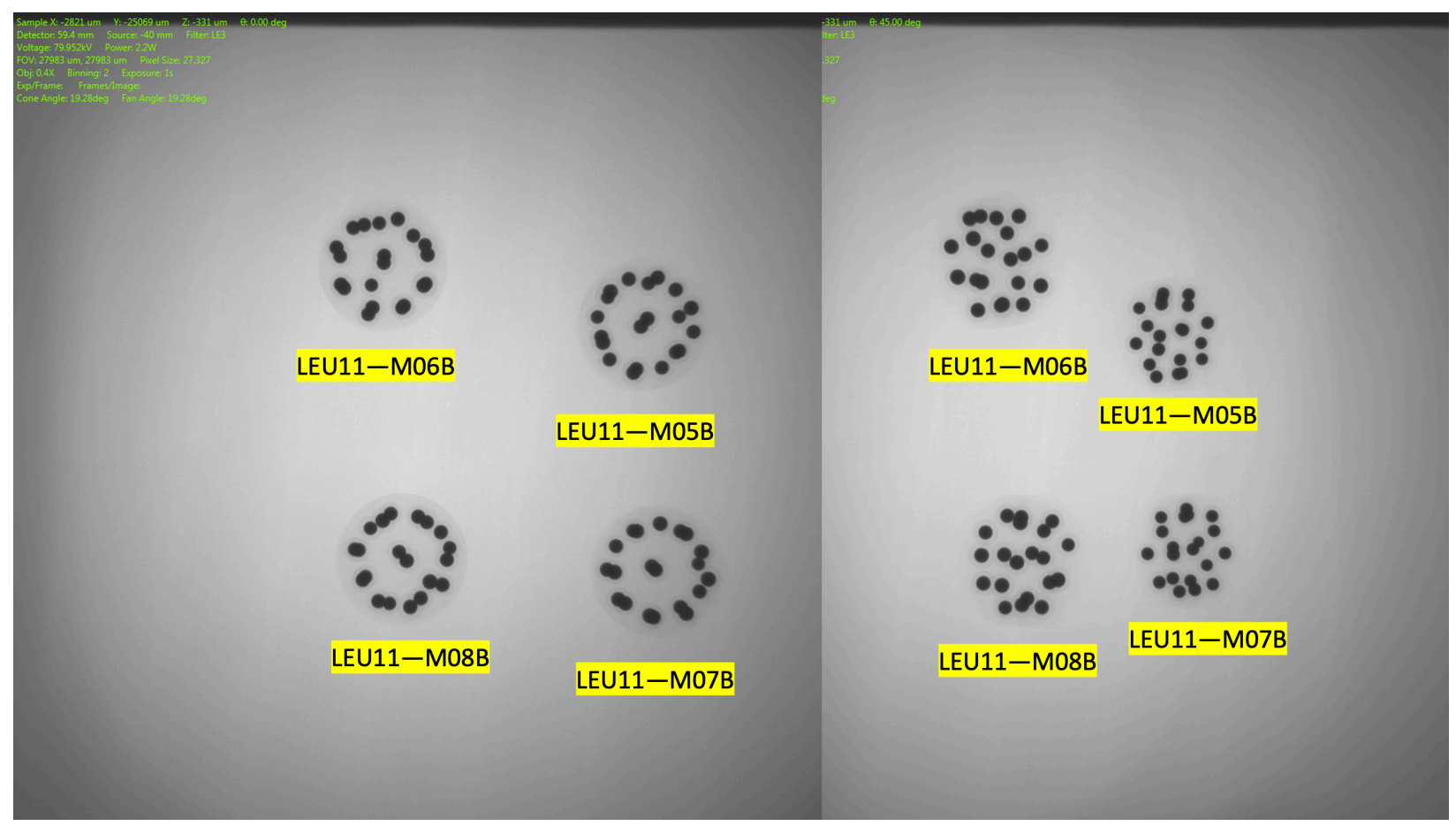

Figure A-10. Radiography sets of LEU11-M05B-LEU11-M08B; axial image orientation (left) and $45^{\circ}$ tilt from the axial direction (right). 


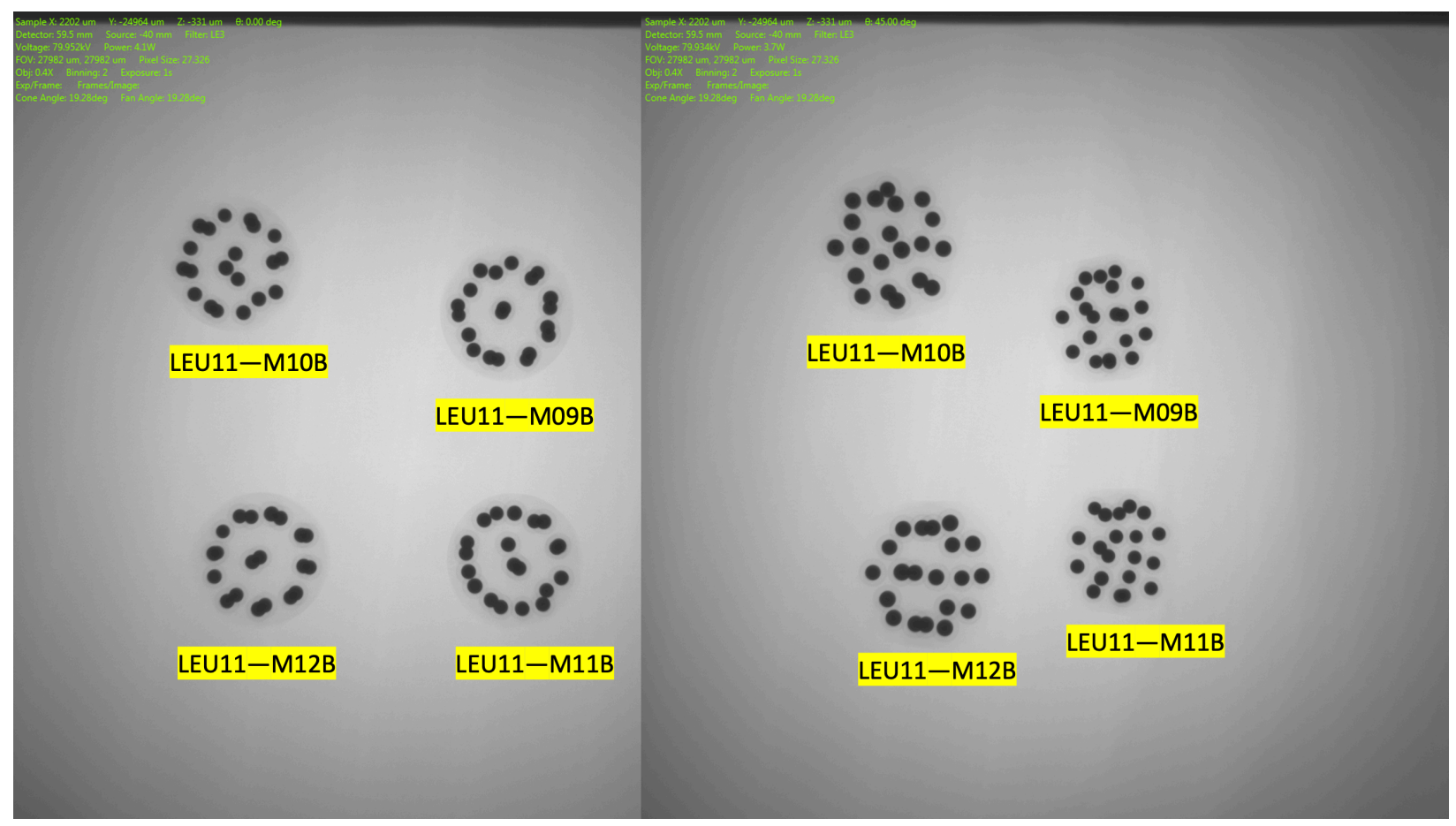

Figure A-11. Radiography sets of LEU11-M09B-LEU11-M12B; axial image orientation (left) and $45^{\circ}$ tilt from the axial direction (right).

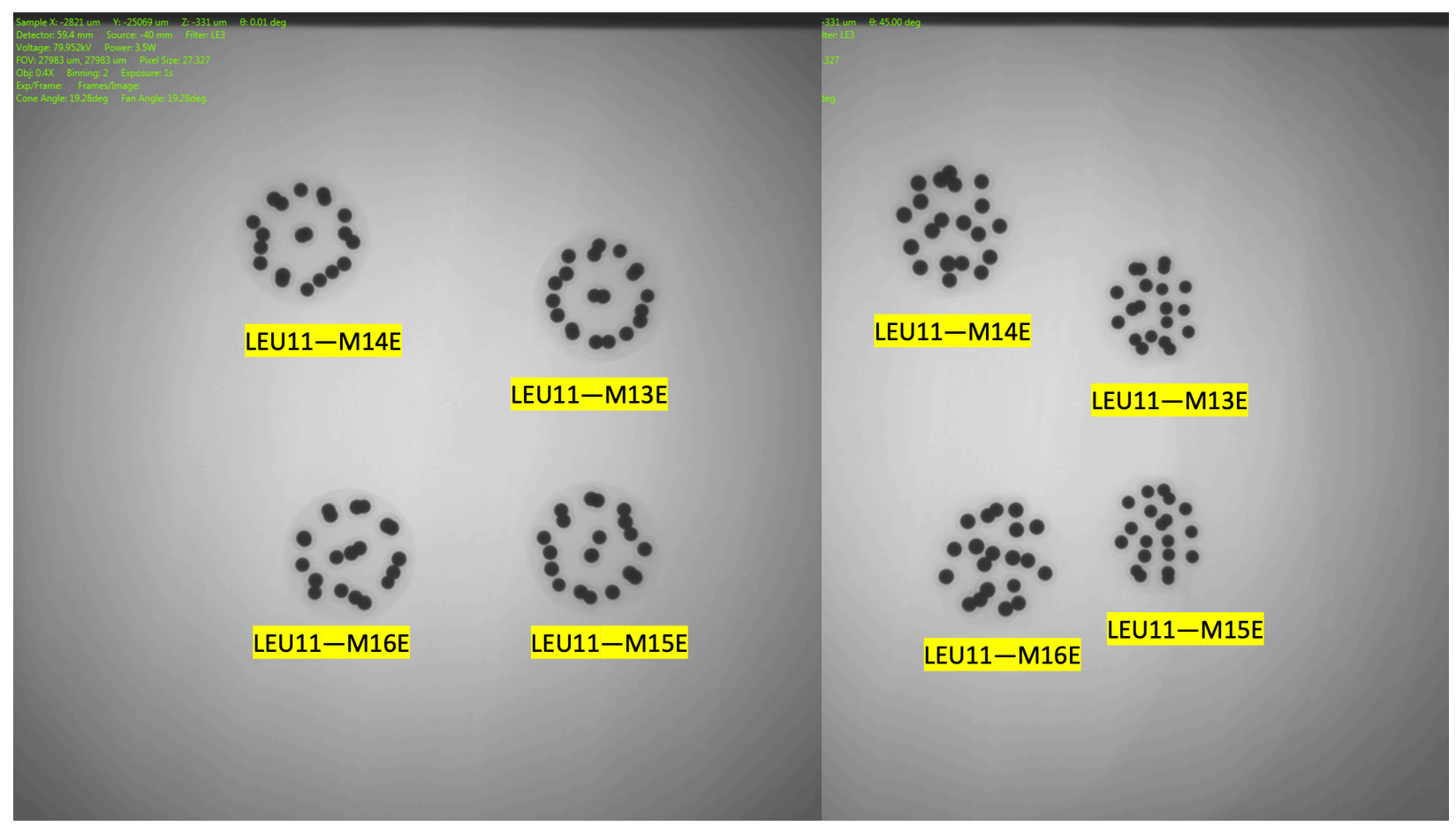

Figure A-12. Radiography sets of LEU11-M13B-LEU11-M16B; axial image orientation (left) and $45^{\circ}$ tilt from the axial direction (right). 


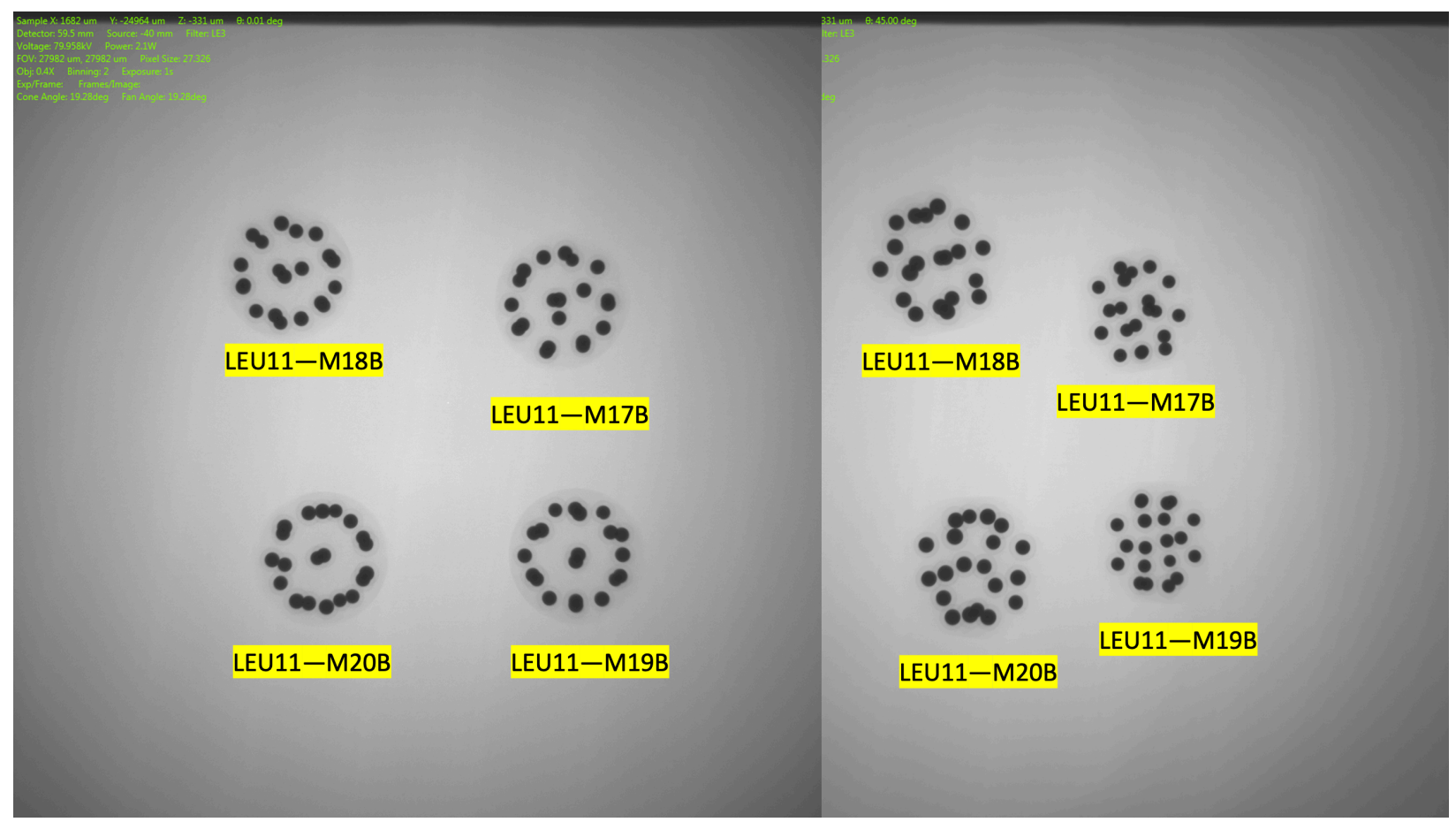

Figure A-13. Radiography sets of LEU11-M17B-LEU11-M20B; axial image orientation (left) and $45^{\circ}$ tilt from the axial direction (right).

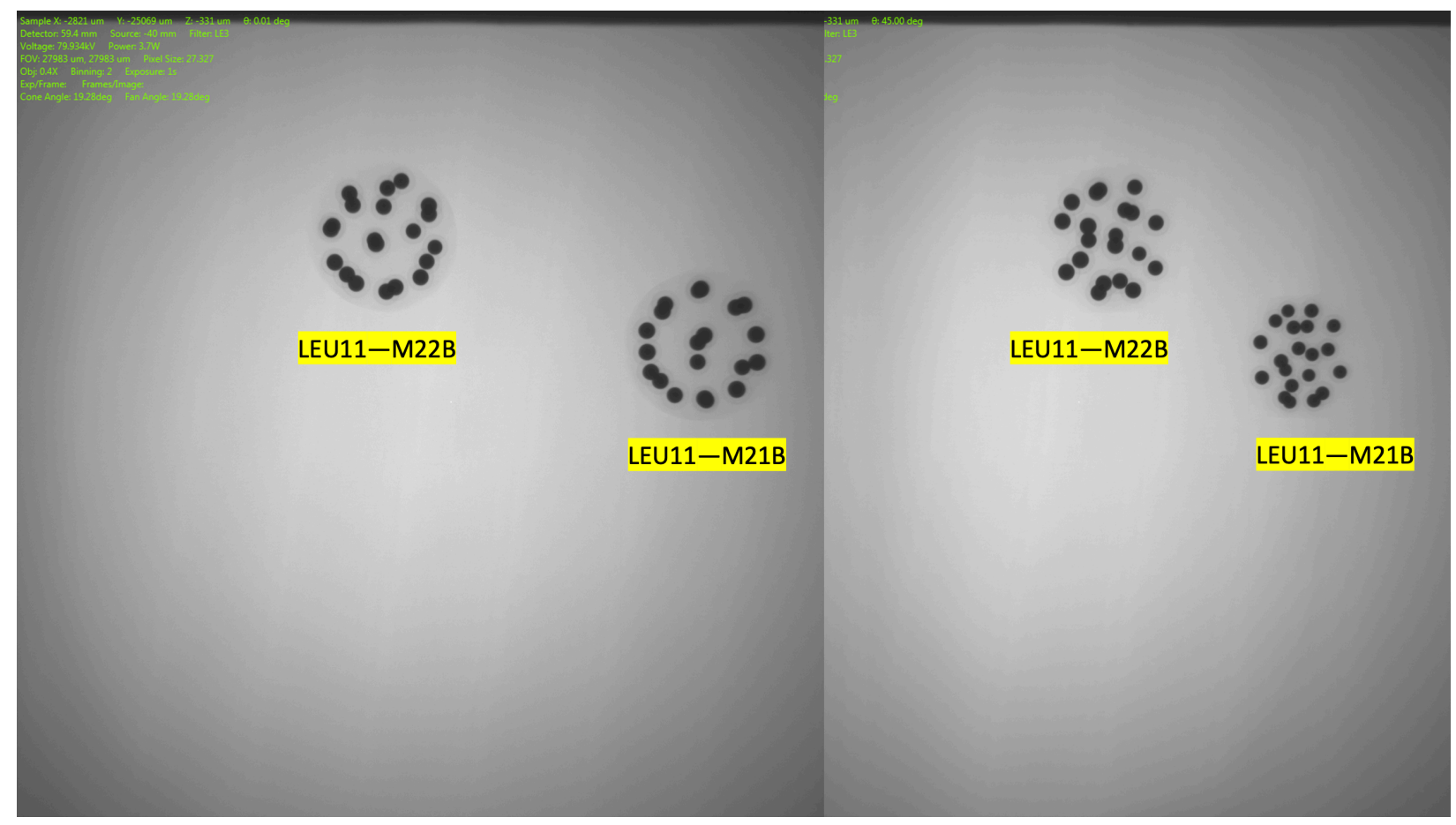

Figure A-14. Radiography sets of LEU11-M21B-LEU11-M22B; axial image orientation (left) and $45^{\circ}$ tilt from the axial direction (right). 


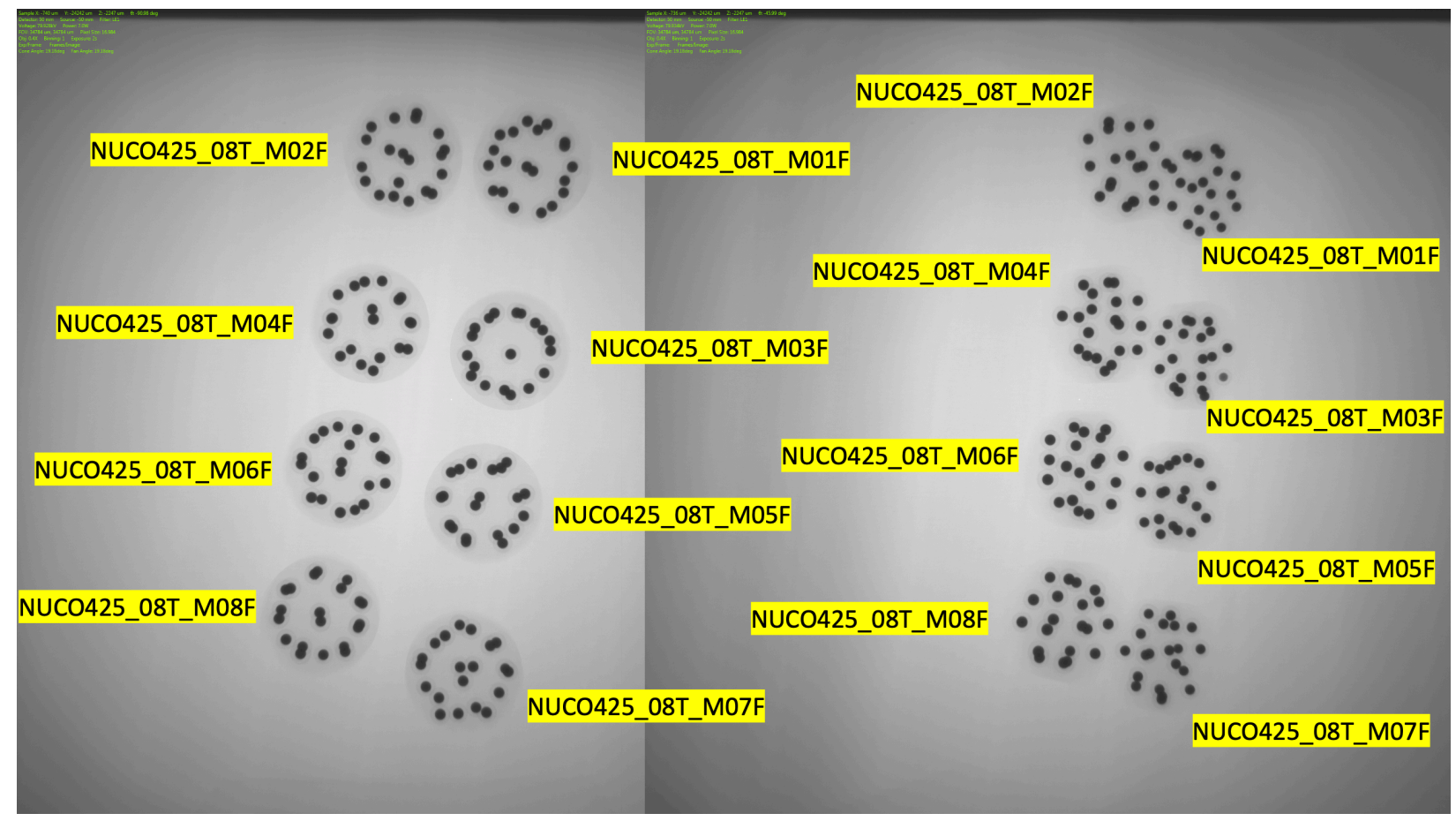

Figure A-15. Radiography sets of NUCO425-08T-M01F- NUCO425-08T-M08F; axial image orientation (left) and $45^{\circ}$ tilt from the axial direction (right). 


\section{APPENDIX B.}

Table B-1. XCT data for particles in compact NUCO-M01F.

\begin{tabular}{|c|c|c|c|c|c|c|c|c|}
\hline \multicolumn{3}{|c|}{ Centroid $(\boldsymbol{\mu m})$} & \multicolumn{2}{c|}{ Volume $\left(\mathbf{m m}^{\mathbf{3}}\right)$} & \multirow{2}{*}{ Surface distance $(\boldsymbol{\mu m})$} & \multicolumn{3}{c|}{ Nearest neighbor $(\boldsymbol{\mu m})$} \\
\cline { 9 - 10 } & $\boldsymbol{y}$ & $\boldsymbol{y}$ & Kernel & Region & & 1st & 2nd & 3rd \\
\hline 237 & 1,420 & 506 & 0.0437 & 1.895 & 449 & 1,042 & 1,070 & 1,263 \\
\hline$-1,342$ & 265 & 555 & 0.0425 & 2.058 & 495 & 986 & 1,142 & 1,220 \\
\hline 34 & -277 & 557 & 0.0410 & 2.461 & 501 & 1,379 & 1,478 & 1,493 \\
\hline$-1,083$ & $-1,260$ & 680 & 0.0430 & 1.889 & 622 & 927 & 1,050 & 1,222 \\
\hline 1,298 & 759 & 683 & 0.0457 & 2.438 & 628 & 1,158 & 1,263 & 1,277 \\
\hline-788 & 1,324 & 797 & 0.0441 & 2.174 & 686 & 1,029 & 1,070 & 1,220 \\
\hline 1,429 & -810 & 811 & 0.0436 & 2.499 & 669 & 1,033 & 1,060 & 1,253 \\
\hline 7 & $-1,764$ & 909 & 0.0422 & 2.350 & 582 & 1,127 & 1,220 & 1,222 \\
\hline$-1,548$ & -555 & 1,062 & 0.0420 & 1.591 & 659 & 927 & 958 & 986 \\
\hline 1,694 & -30 & 1,433 & 0.0458 & 1.713 & 587 & 902 & 1,033 & 1,157 \\
\hline 581 & 1,537 & 1,482 & 0.0441 & 2.160 & 580 & 963 & 1,042 & 1,326 \\
\hline 873 & $-1,375$ & 1,516 & 0.0409 & 1.853 & 708 & 984 & 1,060 & 1,080 \\
\hline$-1,566$ & 419 & 1,664 & 0.0409 & 2.318 & 645 & 1,142 & 1,145 & 1,146 \\
\hline-819 & $-1,329$ & 1,695 & 0.0426 & 1.969 & 773 & 979 & 1,050 & 1,080 \\
\hline-794 & 1,394 & 1,823 & 0.0407 & 2.501 & 623 & 1,029 & 1,254 & 1,424 \\
\hline 91 & 38 & 1,898 & 0.0467 & 2.907 & 651 & 1,379 & 1,527 & 1,542 \\
\hline 1,268 & 1,032 & 1,930 & 0.0436 & 2.102 & 606 & 963 & 1,248 & 1,277 \\
\hline$-1,469$ & -669 & 2,011 & 0.0452 & 1.808 & 541 & 958 & 979 & 1,146 \\
\hline 1,451 & -639 & 2,052 & 0.0423 & 1.899 & 501 & 902 & 1,079 & 1,253 \\
\hline 133 & $-1,611$ & 2,119 & 0.0394 & 1.799 & 432 & 984 & 1,079 & 1,227 \\
\hline
\end{tabular}


Table B-2. XCT data for particles in compact NUCO-M02F.

\begin{tabular}{|c|c|c|c|c|c|c|c|c|}
\hline \multicolumn{3}{|c|}{ Centroid $(\boldsymbol{\mu m})$} & \multicolumn{2}{c|}{ Volume $\left(\mathbf{m m}^{\mathbf{3}}\right)$} & \multirow{3}{*}{ Surface distance $(\boldsymbol{\mu m})$} & \multicolumn{3}{c|}{ Nearest neighbor $(\boldsymbol{\mu m})$} \\
\hline $\boldsymbol{y n n n n n n n n n}$\cline { 8 - 9 } & $\boldsymbol{y}$ & $\boldsymbol{z}$ & Kernel & Region & & $\mathbf{1 s t}$ & $\mathbf{2 n d}$ & 3rd \\
\hline$-1,507$ & -344 & 785 & 0.0387 & 2.011 & 476 & 940 & 1,151 & 1,359 \\
\hline-848 & $-1,699$ & 778 & 0.0379 & 2.169 & 476 & 1,035 & 1,284 & 1,397 \\
\hline-50 & -194 & 821 & 0.0388 & 2.117 & 510 & 851 & 1,378 & 1,465 \\
\hline-753 & 1,162 & 834 & 0.0373 & 1.849 & 532 & 936 & 1,145 & 1,147 \\
\hline 1,197 & 894 & 862 & 0.0357 & 2.153 & 555 & 934 & 1,228 & 1,424 \\
\hline 1,067 & $-1,569$ & 893 & 0.0346 & 1.986 & 589 & 1,008 & 1104 & 1,180 \\
\hline 1,618 & -623 & 1,040 & 0.0396 & 2.356 & 638 & 1,104 & 1,194 & 1,328 \\
\hline 78 & 1,206 & 1,262 & 0.0393 & 2.028 & 770 & 936 & 1,060 & 1,137 \\
\hline$-1,419$ & 394 & 1,361 & 0.0398 & 2.185 & 695 & 940 & 1,145 & 1165 \\
\hline 260 & $-1,907$ & 1,393 & 0.0410 & 2.035 & 682 & 1,008 & 1,075 & 1,198 \\
\hline 381 & -477 & 1,498 & 0.0437 & 1.590 & 1,178 & 851 & 886 & 1,326 \\
\hline$-1,359$ & $-1,207$ & 1,532 & 0.0430 & 1.777 & 663 & 900 & 1,035 & 1,151 \\
\hline 1,439 & 538 & 1,691 & 0.0452 & 1.847 & 610 & 934 & 1,035 & 1,113 \\
\hline-803 & 1,166 & 1,980 & 0.0402 & 2.495 & 603 & 1,137 & 1,147 & 1,165 \\
\hline 1,181 & $-1,540$ & 2,067 & 0.0431 & 2.228 & 591 & 1,180 & 1,198 & 1,229 \\
\hline$-1,740$ & -618 & 2,096 & 0.0419 & 2.109 & 520 & 900 & 1,292 & 1,359 \\
\hline-530 & $-1,904$ & 2,123 & 0.0428 & 2.218 & 555 & 1,075 & 1,234 & 1,397 \\
\hline 661 & 1,189 & 2,148 & 0.0442 & 2.179 & 521 & 1,060 & 1,113 & 1,424 \\
\hline-199 & -445 & 2,167 & 0.0415 & 2.283 & 510 & 886 & 1,378 & 1,497 \\
\hline 1,500 & -361 & 2,199 & 0.0436 & 1.980 & 476 & 1,035 & 1,194 & 1,229 \\
\hline
\end{tabular}


Table B-3. XCT data for particles in compact NUCO-M04F.

\begin{tabular}{|c|c|c|c|c|c|c|c|c|}
\hline \multicolumn{3}{|c|}{ Centroid $(\mu \mathrm{m})$} & \multicolumn{2}{|c|}{ Volume $\left(\mathrm{mm}^{3}\right)$} & \multirow{2}{*}{ Surface distance $(\mu \mathrm{m})$} & \multicolumn{3}{|c|}{ Nearest neighbor $(\mu \mathrm{m})$} \\
\hline$x$ & $y$ & $z$ & Kernel & Region & & 1 st & 2nd & 3rd \\
\hline 1,211 & $-1,153$ & 782 & 0.0384 & 1.906 & 453 & 872 & 1,035 & 1,385 \\
\hline 1,052 & 700 & 805 & 0.0364 & 1.980 & 476 & 906 & 961 & 1,309 \\
\hline$-1,139$ & $-1,346$ & 822 & 0.0402 & 2.015 & 498 & 881 & 1,165 & 1,345 \\
\hline 70 & $-1,934$ & 859 & 0.0376 & 2.425 & 532 & 1,214 & 1,296 & 1,345 \\
\hline-712 & 1,159 & 918 & 0.0390 & 2.010 & 589 & 1,082 & 1,171 & 1,222 \\
\hline-45 & -6 & 911 & 0.0380 & 2.310 & 578 & 1,103 & 1,309 & 1,322 \\
\hline$-1,592$ & -324 & 1,152 & 0.0414 & 2.226 & 698 & 877 & 1,165 & 1,193 \\
\hline 1,689 & -589 & 1,245 & 0.0394 & 2.006 & 585 & 872 & 888 & 1,368 \\
\hline 448 & 1,157 & 1,302 & 0.0446 & 1.852 & 728 & 906 & 921 & 1,043 \\
\hline$-1,481$ & 526 & 1,340 & 0.0412 & 1.729 & 575 & 877 & 944 & 1,082 \\
\hline 968 & $-1,683$ & 1,637 & 0.0446 & 2.020 & 637 & 839 & 1,035 & 1,214 \\
\hline$-1,214$ & $-1,645$ & 1,647 & 0.0415 & 1.946 & 506 & 837 & 881 & 1,460 \\
\hline 1,308 & 751 & 1,730 & 0.0429 & 1.884 & 591 & 839 & 961 & 1,043 \\
\hline-195 & -261 & 1,975 & 0.0428 & 2.328 & 702 & 1,103 & 1,191 & 1,343 \\
\hline-617 & 1,092 & 2,084 & 0.0441 & 2.173 & 589 & 1,171 & 1,231 & 1,273 \\
\hline 1,463 & -517 & 2,100 & 0.0410 & 2.520 & 578 & 888 & 1,330 & 1,349 \\
\hline 276 & $-1,677$ & 2,113 & 0.0396 & 2.482 & 555 & 839 & 1,296 & 1,466 \\
\hline 699 & 1,104 & 2,186 & 0.0424 & 1.763 & 487 & 839 & 921 & 1,320 \\
\hline$-1,438$ & 185 & 2,219 & 0.0460 & 1.858 & 453 & 944 & 1,193 & 1,231 \\
\hline$-1,047$ & $-1,056$ & 2,218 & 0.0471 & 1.871 & 453 & 837 & 1,191 & 1,302 \\
\hline
\end{tabular}


Table B-4. XCT data for particles in compact NUCO-M05F.

\begin{tabular}{|c|c|c|c|c|c|c|c|c|}
\hline \multicolumn{3}{|c|}{ Centroid $(\boldsymbol{\mu m})$} & \multicolumn{2}{c|}{ Volume $\left(\mathbf{m m}^{\mathbf{3}}\right)$} & \multirow{2}{*}{ Surface distance $(\boldsymbol{\mu m})$} & \multicolumn{3}{c|}{ Nearest neighbor $(\boldsymbol{\mu m})$} \\
\hline $\boldsymbol{y n n n n n n n n n}$\cline { 8 - 9 } & $\boldsymbol{y}$ & $\boldsymbol{z}$ & Kernel & Region & & 1st & 2nd & 3rd \\
\hline 968 & 1,121 & 503 & 0.0421 & 1.881 & 443 & 1,079 & 1,145 & 1,265 \\
\hline-525 & 1,348 & 522 & 0.0401 & 1.865 & 466 & 1,043 & 1,049 & 1,335 \\
\hline$-1,490$ & -320 & 641 & 0.0401 & 2.275 & 582 & 882 & 1,076 & 1,222 \\
\hline 1,114 & $-1,280$ & 713 & 0.0423 & 2.198 & 628 & 843 & 1,058 & 1,545 \\
\hline 1,594 & 188 & 724 & 0.0410 & 2.210 & 660 & 1,030 & 1,145 & 1,248 \\
\hline$-1,352$ & 742 & 742 & 0.0446 & 1.205 & 685 & 293 & 1,049 & 1,076 \\
\hline-578 & $-1,625$ & 755 & 0.0400 & 2.267 & 612 & 872 & 1,031 & 1,512 \\
\hline-43 & 112 & 815 & 0.0452 & 2.814 & 760 & 1,270 & 1,359 & 1,454 \\
\hline$-1,575$ & 552 & 737 & 0.0002 & 0.977 & 582 & 293 & 882 & 1,223 \\
\hline 1,380 & -677 & 1,239 & 0.0434 & 1.641 & 762 & 843 & 859 & 1,030 \\
\hline 321 & $-1,732$ & 1,248 & 0.0431 & 1.820 & 575 & 909 & 1,031 & 1,058 \\
\hline 513 & 1,621 & 1,343 & 0.0430 & 1.844 & 518 & 973 & 1,079 & 1,143 \\
\hline$-1,031$ & $-1,522$ & 1,493 & 0.0441 & 1.727 & 491 & 870 & 872 & 1,353 \\
\hline-405 & 1,376 & 1,558 & 0.0468 & 2.003 & 781 & 973 & 994 & 1,043 \\
\hline 1,363 & 942 & 1,692 & 0.0441 & 2.288 & 579 & 1,139 & 1,143 & 1,248 \\
\hline$-1,701$ & -68 & 1,818 & 0.0453 & 1.992 & 573 & 1,072 & 1,173 & 1,222 \\
\hline$-1,217$ & 887 & 1,856 & 0.0453 & 2.150 & 691 & 994 & 1,072 & 1,131 \\
\hline 320 & 561 & 1,946 & 0.0434 & 2.255 & 599 & 1,139 & 1,158 & 1,234 \\
\hline 190 & $-1,232$ & 1,996 & 0.0399 & 2.402 & 547 & 909 & 1,173 & 1,353 \\
\hline 1,474 & -551 & 2,084 & 0.0441 & 2.231 & 461 & 859 & 1,456 & 1,547 \\
\hline
\end{tabular}


Table B-5. XCT data for particles in compact NUCO-M06F.

\begin{tabular}{|c|c|c|c|c|c|c|c|c|}
\hline \multicolumn{3}{|c|}{ Centroid $(\boldsymbol{\mu m})$} & \multicolumn{2}{c|}{ Volume $\left(\mathbf{m m}^{\mathbf{3}}\right)$} & \multirow{3}{*}{ Surface distance $(\boldsymbol{\mu m})$} & \multicolumn{3}{c|}{ Nearest neighbor $(\boldsymbol{\mu m})$} \\
\hline $\boldsymbol{y n n n n n n n n n}$\cline { 8 - 9 } & $\boldsymbol{y}$ & $\boldsymbol{z}$ & Kernel & Region & & 1st & 2nd & 3rd \\
\hline 452 & 1,587 & 507 & 0.0444 & 1.627 & 449 & 869 & 1,065 & 1,342 \\
\hline$-1,218$ & 871 & 521 & 0.0445 & 1.963 & 466 & 1,074 & 1,115 & 1,214 \\
\hline 1628 & 410 & 605 & 0.0417 & 2.133 & 547 & 1,073 & 1,299 & 1,333 \\
\hline$-1,254$ & -818 & 644 & 0.0402 & 2.079 & 587 & 938 & 953 & 1,134 \\
\hline 162 & $-1,660$ & 700 & 0.0427 & 2.043 & 645 & 977 & 1,005 & 1,254 \\
\hline 156 & -93 & 717 & 0.0423 & 3.215 & 656 & 1,471 & 1,560 & 1,567 \\
\hline 1,263 & $-1,060$ & 719 & 0.0306 & 2.203 & 662 & 1,065 & 1,254 & 1,274 \\
\hline-351 & 1,500 & 829 & 0.0419 & 1.767 & 683 & 869 & 1,065 & 1,115 \\
\hline$-1,557$ & -6 & 1,039 & 0.0433 & 1.860 & 727 & 921 & 953 & 1,074 \\
\hline-686 & $-1,366$ & 1,152 & 0.0430 & 1.631 & 814 & 899 & 924 & 938 \\
\hline 946 & 1,246 & 1,387 & 0.0407 & 1.748 & 669 & 893 & 955 & 1,065 \\
\hline 1,606 & -314 & 1,396 & 0.0454 & 1.791 & 665 & 1,041 & 1,063 & 1,065 \\
\hline 500 & $-1,583$ & 1,613 & 0.0447 & 1.712 & 687 & 954 & 960 & 977 \\
\hline$-1,057$ & 1,109 & 1,701 & 0.0505 & 2.187 & 707 & 976 & 1,188 & 1,200 \\
\hline$-1,254$ & -975 & 1,767 & 0.0395 & 2.126 & 656 & 924 & 1,071 & 1,134 \\
\hline 98 & 1,422 & 1,791 & 0.0449 & 2.384 & 633 & 955 & 1,065 & 1,200 \\
\hline 1,357 & 610 & 1,859 & 0.0462 & 2.281 & 564 & 893 & 1,063 & 1,299 \\
\hline$-1,509$ & 271 & 1,916 & 0.0426 & 2.006 & 507 & 921 & 976 & 1,280 \\
\hline-383 & $-1,560$ & 1,976 & 0.0457 & 1.744 & 449 & 899 & 954 & 1,071 \\
\hline 1,222 & $-1,077$ & 1,992 & 0.0422 & 1.911 & 432 & 960 & 1,041 & 1,274 \\
\hline
\end{tabular}


Table B-6. XCT data for particles in compact NUCO-M07F.

\begin{tabular}{|c|c|c|c|c|c|c|c|c|}
\hline \multicolumn{3}{|c|}{ Centroid $(\boldsymbol{\mu m})$} & \multicolumn{2}{c|}{ Volume $\left(\mathbf{m m}^{\mathbf{3}}\right)$} & \multirow{3}{*}{ Surface distance $(\boldsymbol{\mu m})$} & \multicolumn{3}{c|}{ Nearest neighbor $(\boldsymbol{\mu m})$} \\
\hline $\boldsymbol{y n n n n n n n n n}$\cline { 8 - 9 } & $\boldsymbol{y}$ & $\boldsymbol{z}$ & Kernel & Region & & 1st & 2nd & 3rd \\
\hline$-1,122$ & 765 & 775 & 0.0388 & 2.314 & 442 & 959 & 1,145 & 1,607 \\
\hline$-1,441$ & -947 & 772 & 0.0357 & 1.655 & 442 & 877 & 997 & 1,172 \\
\hline 407 & 12 & 851 & 0.0369 & 2.137 & 521 & 844 & 1,326 & 1,330 \\
\hline-125 & $-2,026$ & 857 & 0.0382 & 1.787 & 532 & 1,102 & 1,102 & 1,247 \\
\hline 1,060 & $-1,637$ & 889 & 0.0388 & 1.898 & 566 & 980 & 1,247 & 1,257 \\
\hline 1,758 & -601 & 1,034 & 0.0377 & 2.366 & 503 & 1,209 & 1,211 & 1,257 \\
\hline-102 & -598 & 1,135 & 0.0362 & 1.870 & 804 & 844 & 1,080 & 1,331 \\
\hline$-1,573$ & -196 & 1,205 & 0.0404 & 1.967 & 711 & 877 & 983 & 1,145 \\
\hline 598 & 1,232 & 1,346 & 0.0402 & 3.090 & 598 & 924 & 1,278 & 1330 \\
\hline$-1,007$ & $-1,614$ & 1,373 & 0.0413 & 1.693 & 661 & 997 & 1,024 & 1,041 \\
\hline 578 & $-1,910$ & 1,698 & 0.0434 & 1.710 & 612 & 980 & 1,064 & 1,068 \\
\hline$-1,113$ & 868 & 1,729 & 0.0453 & 2.320 & 646 & 913 & 959 & 1,271 \\
\hline$-1,387$ & -826 & 1,937 & 0.0436 & 2.730 & 736 & 983 & 1,041 & 1,172 \\
\hline 1,585 & 160 & 1,960 & 0.0408 & 2.450 & 627 & 1,158 & 1,211 & 1,279 \\
\hline-92 & -71 & 2,077 & 0.0399 & 2.101 & 600 & 1,080 & 1,130 & 1,326 \\
\hline 1,464 & $-1,106$ & 2,092 & 0.0438 & 1.876 & 578 & 920 & 1,209 & 1,260 \\
\hline-424 & 1,332 & 2,106 & 0.0470 & 1.948 & 563 & 913 & 1,278 & 1,387 \\
\hline-401 & $-1,982$ & 2,111 & 0.0472 & 1.929 & 566 & 1,024 & 1,064 & 1,285 \\
\hline 939 & 1,092 & 2,194 & 0.0416 & 1.731 & 476 & 924 & 1,158 & 1,387 \\
\hline 562 & -981 & 2,223 & 0.0442 & 1.508 & 453 & 920 & 1,068 & 1,130 \\
\hline
\end{tabular}


Table B-7. XCT data for particles in compact LEU09-M01E.

\begin{tabular}{|c|c|c|c|c|c|c|c|c|}
\hline \multicolumn{3}{|c|}{ Centroid $(\boldsymbol{\mu m})$} & \multicolumn{2}{c|}{ Volume $\left(\mathbf{m m}^{\mathbf{3}}\right)$} & \multirow{2}{*}{ Surface distance $(\boldsymbol{\mu m})$} & \multicolumn{3}{c|}{ Nearest neighbor $(\boldsymbol{\mu m})$} \\
\hline $\boldsymbol{y n n n n n n n n n}$ & $\boldsymbol{y}$ & $\boldsymbol{z}$ & Kernel & Region & & 1st & 2nd & 3rd \\
\hline 674 & 1,132 & 787 & 0.0446 & 2.054 & 464 & 1,075 & 1,090 & 1,355 \\
\hline$-1,346$ & -264 & 785 & 0.0429 & 1.998 & 453 & 1,076 & 1,103 & 1,180 \\
\hline$-1,086$ & 887 & 793 & 0.0453 & 1.933 & 464 & 1,069 & 1,180 & 1,274 \\
\hline-507 & $-1,736$ & 927 & 0.0433 & 2.293 & 600 & 1,000 & 1,083 & 1,332 \\
\hline 1,086 & $-1,662$ & 974 & 0.0455 & 2.215 & 598 & 1,018 & 1,221 & 1,328 \\
\hline 120 & -379 & 998 & 0.0430 & 2.563 & 668 & 1,129 & 1,485 & 1,497 \\
\hline 1,629 & -450 & 997 & 0.0454 & 2.372 & 668 & 1,150 & 1,207 & 1,328 \\
\hline$-1,402$ & $-1,227$ & 1261 & 0.0484 & 2.107 & 647 & 1,045 & 1,076 & 1,083 \\
\hline-249 & 1,307 & 1309 & 0.0441 & 1.904 & 635 & 1,069 & 1,075 & 1,115 \\
\hline 1,371 & 586 & 1423 & 0.0494 & 2.151 & 644 & 1,063 & 1,090 & 1,150 \\
\hline 271 & $-1,921$ & 1528 & 0.0461 & 1.784 & 702 & 969 & 1,000 & 1,018 \\
\hline$-1,606$ & 217 & 1743 & 0.0456 & 2.037 & 605 & 1,078 & 1,078 & 1,103 \\
\hline 1,441 & $-1,098$ & 1998 & 0.0440 & 2.107 & 669 & 1,029 & 1,075 & 1,207 \\
\hline-968 & 1,006 & 2107 & 0.0454 & 2.356 & 647 & 1,078 & 1,115 & 1,324 \\
\hline-65 & -284 & 2107 & 0.0417 & 2.579 & 679 & 1,129 & 1,411 & 1,466 \\
\hline 524 & 1,196 & 2132 & 0.0414 & 2.619 & 657 & 1,135 & 1,262 & 1,355 \\
\hline$-1,452$ & -752 & 2190 & 0.0477 & 2.203 & 600 & 1,045 & 1,078 & 1,286 \\
\hline-756 & $-1,833$ & 2232 & 0.0428 & 2.203 & 555 & 1,248 & 1,286 & 1,315 \\
\hline 1,497 & -57 & 2259 & 0.0474 & 2.243 & 521 & 1,063 & 1,075 & 1,329 \\
\hline 572 & $-1,518$ & 2356 & 0.0416 & 1.940 & 430 & 969 & 1,029 & 1,370 \\
\hline
\end{tabular}


Table B-8. XCT data for particles in compact LEU09-M07E.

\begin{tabular}{|c|c|c|c|c|c|c|c|c|}
\hline \multicolumn{3}{|c|}{ Centroid $(\boldsymbol{\mu m})$} & \multicolumn{2}{c|}{ Volume $\left(\mathbf{m m}^{\mathbf{3}}\right)$} & \multirow{2}{*}{ Surface distance $(\boldsymbol{\mu m})$} & \multicolumn{3}{c|}{ Nearest neighbor $(\boldsymbol{\mu m})$} \\
\hline $\boldsymbol{y n n n n n n n y y}$ & $\boldsymbol{y}$ & $\boldsymbol{z}$ & Kernel & Region & & $\mathbf{1 s t}$ & $\mathbf{2 n d}$ & 3rd \\
\hline 24 & 1,188 & 794 & 0.0441 & 2.074 & 453 & 1,100 & 1,112 & 1,341 \\
\hline 1,237 & 477 & 797 & 0.0411 & 2.206 & 453 & 1,067 & 1,145 & 1,372 \\
\hline$-1,405$ & 279 & 905 & 0.0441 & 2.093 & 566 & 962 & 1,004 & 1,297 \\
\hline 1,356 & $-1,255$ & 921 & 0.0441 & 2.151 & 577 & 969 & 1,039 & 1,337 \\
\hline-132 & -315 & 980 & 0.0420 & 2.591 & 645 & 1,157 & 1,407 & 1,521 \\
\hline$-1,296$ & $-1,294$ & 996 & 0.0453 & 2.455 & 657 & 1,034 & 1,107 & 1,327 \\
\hline 168 & $-2,031$ & 1,015 & 0.0432 & 2.352 & 612 & 981 & 1,162 & 1,300 \\
\hline 1,640 & -438 & 1,357 & 0.0464 & 1.918 & 657 & 969 & 1,052 & 1,145 \\
\hline-888 & 998 & 1,378 & 0.0428 & 1.976 & 688 & 1,004 & 1,095 & 1,100 \\
\hline$-1,650$ & -399 & 1,542 & 0.0402 & 1.721 & 650 & 962 & 1,050 & 1,067 \\
\hline 823 & 1,094 & 1,562 & 0.0459 & 2.018 & 639 & 1,067 & 1,092 & 1,098 \\
\hline 819 & $-1,720$ & 1,680 & 0.0487 & 1.751 & 713 & 981 & 983 & 1,039 \\
\hline-750 & $-1,824$ & 1,696 & 0.0467 & 1.897 & 658 & 1,034 & 1,048 & 1,134 \\
\hline-114 & 1,189 & 2,128 & 0.0462 & 2.461 & 600 & 1,095 & 1,098 & 1,341 \\
\hline-51 & -323 & 2,134 & 0.0440 & 2.545 & 600 & 1,157 & 1,514 & 1,520 \\
\hline 1,372 & 361 & 2,158 & 0.0422 & 2.438 & 566 & 1,092 & 1,163 & 1,372 \\
\hline$-1,433$ & 394 & 2,196 & 0.0480 & 2.213 & 532 & 1,050 & 1,153 & 1,297 \\
\hline 1,483 & $-1,005$ & 2,228 & 0.0474 & 2.126 & 498 & 1,052 & 1,119 & 1,337 \\
\hline$-1,356$ & $-1,083$ & 2,306 & 0.0455 & 2.031 & 419 & 1,067 & 1,134 & 1,327 \\
\hline 93 & $-1,930$ & 2,309 & 0.0438 & 1.880 & 419 & 983 & 1,048 & 1,300 \\
\hline
\end{tabular}


Table B-9. XCT data for particles in compact LEU09-M14E.

\begin{tabular}{|c|c|c|c|c|c|c|c|c|}
\hline \multicolumn{3}{|c|}{ Centroid $(\boldsymbol{\mu m})$} & \multicolumn{2}{c|}{ Volume $\left(\mathbf{m m}^{\mathbf{3}}\right)$} & \multirow{2}{*}{ Surface distance $(\boldsymbol{\mu m})$} & \multicolumn{3}{c|}{ Nearest neighbor $(\boldsymbol{\mu m})$} \\
\hline $\boldsymbol{y n n n n n n n n n}$\cline { 8 - 9 } & $\boldsymbol{y}$ & $\boldsymbol{z}$ & Kernel & Region & & 1st & 2nd & 3rd \\
\hline 38 & 1,048 & 791 & 0.0443 & 2.001 & 464 & 1,056 & 1,141 & 1,167 \\
\hline 1,150 & 802 & 862 & 0.0431 & 2.151 & 532 & 1,141 & 1,142 & 1,223 \\
\hline$-1,645$ & -213 & 869 & 0.0409 & 2.289 & 544 & 1,179 & 1,209 & 1,252 \\
\hline 276 & -446 & 957 & 0.0455 & 2.406 & 623 & 1,178 & 1,247 & 1,345 \\
\hline 1,528 & -937 & 995 & 0.0405 & 2.244 & 656 & 994 & 1,108 & 1,345 \\
\hline$-1,100$ & $-1,673$ & 1,042 & 0.0472 & 2.471 & 571 & 1,150 & 1,152 & 1,438 \\
\hline$-1,080$ & 884 & 1,082 & 0.0472 & 2.375 & 665 & 1,090 & 1,167 & 1,252 \\
\hline 302 & $-1,991$ & 1,081 & 0.0408 & 2.616 & 626 & 1,175 & 1,282 & 1,333 \\
\hline 1,726 & 38 & 1,484 & 0.0482 & 1.800 & 536 & 1,054 & 1,108 & 1,142 \\
\hline-752 & -671 & 1,487 & 0.0425 & 1.773 & 1155 & 1,150 & 1,172 & 1,178 \\
\hline 1,210 & $-1,568$ & 1,694 & 0.0501 & 1.840 & 585 & 994 & 1,068 & 1,175 \\
\hline 145 & 1,370 & 1,791 & 0.0466 & 2.286 & 582 & 1,056 & 1,113 & 1,168 \\
\hline-540 & $-1,835$ & 2,035 & 0.0486 & 2.152 & 716 & 1,037 & 1,152 & 1,256 \\
\hline$-1,609$ & 64 & 2,046 & 0.0478 & 2.277 & 649 & 1,158 & 1,209 & 1,212 \\
\hline 1,144 & 841 & 2,084 & 0.0457 & 2.457 & 657 & 1,159 & 1,168 & 1,223 \\
\hline-845 & 1,001 & 2,141 & 0.0469 & 2.407 & 691 & 1,090 & 1,113 & 1,212 \\
\hline 220 & -254 & 2,188 & 0.0403 & 2.582 & 645 & 1,247 & 1,269 & 1,424 \\
\hline 1,593 & -614 & 2,302 & 0.0427 & 2.139 & 532 & 1,054 & 1,194 & 1,348 \\
\hline$-1,482$ & $-1,054$ & 2,318 & 0.0463 & 2.186 & 510 & 1,158 & 1,172 & 1,256 \\
\hline 432 & $-1,790$ & 2,392 & 0.0506 & 1.987 & 442 & 1,037 & 1,068 & 1,333 \\
\hline
\end{tabular}


Table B-10. XCT data for particles in compact LEU09-M19E.

\begin{tabular}{|c|c|c|c|c|c|c|c|c|}
\hline \multicolumn{3}{|c|}{ Centroid $(\boldsymbol{\mu m})$} & \multicolumn{2}{c|}{ Volume $\left(\mathbf{m m}^{\mathbf{3}}\right)$} & \multirow{2}{*}{ Surface distance $(\boldsymbol{\mu m})$} & \multicolumn{3}{c|}{ Nearest neighbor $(\boldsymbol{\mu m})$} \\
\hline $\boldsymbol{y n n n n n n n n n}$\cline { 8 - 9 } & $\boldsymbol{y}$ & $\boldsymbol{z}$ & Kernel & Region & & 1st & 2nd & 3rd \\
\hline 1,477 & -2 & 790 & 0.0432 & 2.227 & 476 & 951 & 1,354 & 1,423 \\
\hline 562 & 1,087 & 817 & 0.0443 & 2.554 & 498 & 1,253 & 1,361 & 1,423 \\
\hline$-1,341$ & 535 & 827 & 0.0456 & 1.793 & 510 & 872 & 1,031 & 1,305 \\
\hline 86 & -379 & 846 & 0.0431 & 2.229 & 532 & 935 & 1,332 & 1,442 \\
\hline$-1,296$ & $-1,333$ & 908 & 0.0428 & 2.169 & 589 & 1,026 & 1,060 & 1,459 \\
\hline 377 & $-1,952$ & 952 & 0.0439 & 2.131 & 634 & 1,087 & 1,154 & 1,172 \\
\hline 1,357 & $-1,321$ & 1,071 & 0.0445 & 2.416 & 628 & 1,172 & 1,213 & 1,330 \\
\hline-767 & 1,131 & 1,105 & 0.0477 & 2.078 & 658 & 872 & 1,146 & 1,212 \\
\hline$-1,657$ & -377 & 1,188 & 0.0455 & 1.851 & 645 & 969 & 1,031 & 1,060 \\
\hline-619 & $-1,937$ & 1,387 & 0.0468 & 1.808 & 577 & 932 & 1,026 & 1,087 \\
\hline-432 & -355 & 1,623 & 0.0458 & 1.650 & 1053 & 881 & 935 & 1,300 \\
\hline 1,575 & 64 & 1,733 & 0.0458 & 1.907 & 676 & 910 & 951 & 1,134 \\
\hline 79 & 1,369 & 1,938 & 0.0430 & 2.389 & 612 & 1,212 & 1,253 & 1,269 \\
\hline 616 & $-1,792$ & 2,070 & 0.0424 & 2.288 & 600 & 1,154 & 1,162 & 1,330 \\
\hline 1,215 & 822 & 2,086 & 0.0476 & 2.094 & 589 & 910 & 1,269 & 1,425 \\
\hline$-1,194$ & 764 & 2,103 & 0.0437 & 2.360 & 566 & 1,146 & 1,305 & 1,351 \\
\hline$-1,654$ & -505 & 2,148 & 0.0455 & 2.080 & 521 & 969 & 1,338 & 1,351 \\
\hline 257 & -230 & 2,159 & 0.0454 & 1.972 & 510 & 881 & 1,332 & 1,342 \\
\hline 1,393 & -942 & 2,223 & 0.0449 & 1.973 & 453 & 1,134 & 1,162 & 1,213 \\
\hline-719 & $-1,551$ & 2,230 & 0.0424 & 2.146 & 442 & 932 & 1,365 & 1,371 \\
\hline
\end{tabular}


Table B-11. XCT data for particles in compact LEU09-M24E.

\begin{tabular}{|c|c|c|c|c|c|c|c|c|}
\hline \multicolumn{3}{|c|}{ Centroid $(\boldsymbol{\mu m})$} & \multicolumn{2}{c|}{ Volume $\left(\mathbf{m m}^{\mathbf{3}}\right)$} & \multirow{2}{*}{ Surface distance $(\boldsymbol{\mu m})$} & \multicolumn{3}{c|}{ Nearest neighbor $(\boldsymbol{\mu m})$} \\
\hline $\boldsymbol{y n n n n n n n n n}$\cline { 8 - 9 } & $\boldsymbol{y}$ & $\boldsymbol{z}$ & Kernel & Region & & 1st & 2nd & 3rd \\
\hline-916 & 745 & 785 & 0.0415 & 2.221 & 430 & 1,138 & 1,175 & 1,178 \\
\hline 952 & 542 & 862 & 0.0428 & 1.991 & 510 & 1,018 & 1,020 & 1,033 \\
\hline 163 & 1,180 & 949 & 0.0384 & 2.181 & 600 & 1,018 & 1,175 & 1,200 \\
\hline$-1,477$ & -803 & 969 & 0.0427 & 2.247 & 623 & 973 & 1,054 & 1,363 \\
\hline 1,529 & -795 & 1,015 & 0.0464 & 2.226 & 668 & 1,060 & 1,140 & 1,201 \\
\hline 61 & -617 & 1,048 & 0.0443 & 3.096 & 702 & 1,458 & 1,474 & 1,479 \\
\hline-756 & $-1,955$ & 1,073 & 0.0421 & 2.356 & 555 & 1,101 & 1,234 & 1,339 \\
\hline 897 & $-1,807$ & 1,155 & 0.0449 & 2.448 & 612 & 1,107 & 1,201 & 1,281 \\
\hline$-1,587$ & 37 & 1,446 & 0.0467 & 1.971 & 676 & 902 & 973 & 1,178 \\
\hline 1,630 & 139 & 1,508 & 0.0449 & 1.890 & 612 & 959 & 1,020 & 1,060 \\
\hline 956 & 938 & 1817 & 0.0478 & 2.148 & 704 & 991 & 1,033 & 1,091 \\
\hline 46 & $-2,039$ & 1,823 & 0.0487 & 1.746 & 645 & 994 & 1,065 & 1,101 \\
\hline-918 & 964 & 1,902 & 0.0487 & 2.708 & 697 & 1040 & 1,138 & 1,231 \\
\hline$-1,371$ & $-1,280$ & 1,903 & 0.0448 & 1.902 & 666 & 892 & 1,054 & 1,085 \\
\hline 1,448 & $-1,140$ & 2,099 & 0.0475 & 2.149 & 674 & 974 & 1,140 & 1,174 \\
\hline 62 & 1,212 & 2,147 & 0.0434 & 2.496 & 713 & 991 & 1,040 & 1,203 \\
\hline$-1,328$ & -255 & 2,258 & 0.0423 & 2.901 & 611 & 902 & 1,085 & 1,335 \\
\hline 1,115 & -31 & 2,299 & 0.0413 & 2.707 & 566 & 959 & 1,094 & 1,174 \\
\hline-808 & $-1,765$ & 2,398 & 0.0408 & 1.969 & 464 & 892 & 1,065 & 1,339 \\
\hline 746 & $-1,717$ & 2,451 & 0.0457 & 1.976 & 419 & 974 & 994 & 1,308 \\
\hline
\end{tabular}


Table B-12. XCT data for particles in compact LEU09-M26E.

\begin{tabular}{|c|c|c|c|c|c|c|c|c|}
\hline \multicolumn{3}{|c|}{ Centroid $(\boldsymbol{\mu m})$} & \multicolumn{2}{c|}{ Volume $\left(\mathbf{m m}^{\mathbf{3}}\right)$} & \multirow{2}{*}{ Surface distance $(\boldsymbol{\mu m})$} & \multicolumn{3}{c|}{ Nearest neighbor $(\boldsymbol{\mu m})$} \\
\hline $\boldsymbol{y n n n n n n n n n}$\cline { 8 - 9 } & $\boldsymbol{y}$ & $\boldsymbol{z}$ & Kernel & Region & & 1st & 2nd & 3rd \\
\hline$-1,218$ & 679 & 793 & 0.0452 & 2.146 & 464 & 1,094 & 1,144 & 1,359 \\
\hline 230 & 1,103 & 818 & 0.0410 & 2.312 & 487 & 1,124 & 1,196 & 1,380 \\
\hline 60 & -283 & 930 & 0.0464 & 2.398 & 600 & 896 & 1,360 & 1,401 \\
\hline$-1,412$ & $-1,179$ & 987 & 0.0428 & 2.457 & 653 & 1,158 & 1,279 & 1,308 \\
\hline-368 & $-1,975$ & 1,029 & 0.0430 & 2.263 & 620 & 1,013 & 1,134 & 1,314 \\
\hline 1,587 & 7 & 1032 & 0.0445 & 2.385 & 665 & 1,073 & 1,230 & 1,272 \\
\hline 1,443 & $-1,257$ & 1,047 & 0.0497 & 2.334 & 581 & 1,111 & 1,206 & 1,272 \\
\hline 569 & $-1,847$ & 1,394 & 0.0411 & 2.098 & 680 & 1,013 & 1,013 & 1,111 \\
\hline$-1,611$ & -125 & 1,422 & 0.0475 & 2.036 & 668 & 1,046 & 1,094 & 1,158 \\
\hline 1,185 & 882 & 1,505 & 0.0485 & 2.126 & 596 & 1,073 & 1,161 & 1,175 \\
\hline-573 & 1,166 & 1,602 & 0.0445 & 2.012 & 688 & 1,074 & 1,115 & 1,124 \\
\hline 372 & -429 & 1,757 & 0.0469 & 1.763 & 1053 & 875 & 896 & 1,387 \\
\hline-845 & $-1,733$ & 2,028 & 0.0468 & 2.199 & 663 & 1,021 & 1,134 & 1,251 \\
\hline$-1,362$ & 592 & 2,142 & 0.0512 & 2.288 & 641 & 1,046 & 1,115 & 1,282 \\
\hline 323 & 1,258 & 2,187 & 0.0424 & 2.439 & 623 & 1,074 & 1,161 & 1,380 \\
\hline-276 & -85 & 2,234 & 0.0439 & 2.098 & 577 & 875 & 1,282 & 1,360 \\
\hline$-1,519$ & -998 & 2,249 & 0.0466 & 2.065 & 555 & 1,021 & 1,206 & 1,279 \\
\hline 1,458 & $-1,160$ & 2,248 & 0.0496 & 2.104 & 555 & 1,206 & 1,231 & 1,323 \\
\hline 1,566 & 66 & 2,261 & 0.0451 & 2.275 & 544 & 1,175 & 1,230 & 1,231 \\
\hline 347 & $-1,864$ & 2,382 & 0.0436 & 1.981 & 430 & 1,013 & 1,251 & 1,323 \\
\hline
\end{tabular}


Table B-13. XCT data for particles in compact LEU09-M29E.

\begin{tabular}{|c|c|c|c|c|c|c|c|c|}
\hline \multicolumn{3}{|c|}{ Centroid $(\boldsymbol{\mu m})$} & \multicolumn{2}{c|}{ Volume $\left(\mathbf{m m}^{\mathbf{3}}\right)$} & \multirow{3}{*}{ Surface distance $(\boldsymbol{\mu m})$} & \multicolumn{3}{c|}{ Nearest neighbor $(\boldsymbol{\mu m})$} \\
\hline $\boldsymbol{y n n n n n n n n n}$ & $\boldsymbol{y}$ & $\boldsymbol{z}$ & Kernel & Region & & 1st & 2nd & 3rd \\
\hline-404 & 1,157 & 790 & 0.0435 & 2.367 & 487 & 1,053 & 1,066 & 1,346 \\
\hline 1,282 & 525 & 805 & 0.0449 & 2.398 & 498 & 1,014 & 1,067 & 1,338 \\
\hline 1,353 & $-1,112$ & 896 & 0.0416 & 2.524 & 589 & 955 & 1,054 & 1,338 \\
\hline$-1,494$ & -513 & 929 & 0.0420 & 2.086 & 623 & 900 & 982 & 1,021 \\
\hline$-1,489$ & 385 & 993 & 0.0450 & 2.169 & 658 & 900 & 1,252 & 1,264 \\
\hline-96 & $-1,921$ & 1,003 & 0.0413 & 2.514 & 702 & 1,068 & 1,084 & 1,164 \\
\hline$-1,162$ & $-1,462$ & 1,101 & 0.0469 & 2.302 & 673 & 1,021 & 1,145 & 1,164 \\
\hline 590 & 1,168 & 1,174 & 0.0415 & 2.167 & 707 & 1,014 & 1,066 & 1,086 \\
\hline 1,650 & -255 & 1,433 & 0.0454 & 1.938 & 668 & 1,054 & 1,067 & 1,078 \\
\hline 859 & $-1,724$ & 1,438 & 0.0473 & 1.853 & 657 & 955 & 997 & 1,068 \\
\hline-733 & 1,080 & 1,787 & 0.0428 & 2.000 & 729 & 955 & 989 & 1,053 \\
\hline$-1,698$ & -465 & 1,888 & 0.0495 & 1.678 & 605 & 918 & 982 & 1,065 \\
\hline-567 & $-1,894$ & 1,979 & 0.0486 & 1.929 & 631 & 1,063 & 1,084 & 1,111 \\
\hline 27 & -348 & 2,057 & 0.0458 & 3.462 & 736 & 1,521 & 1,548 & 1,568 \\
\hline 1,295 & 574 & 2,142 & 0.0439 & 2.669 & 657 & 1,147 & 1,312 & 1,336 \\
\hline 138 & 1,193 & 2,162 & 0.0468 & 2.336 & 634 & 955 & 1,086 & 1,312 \\
\hline 1,414 & -950 & 2,223 & 0.0437 & 2.409 & 578 & 1,078 & 1,234 & 1,326 \\
\hline$-1,376$ & 485 & 2,248 & 0.0438 & 2.118 & 555 & 989 & 1,065 & 1,264 \\
\hline 434 & $-1,836$ & 2,333 & 0.0445 & 1.918 & 464 & 997 & 1,063 & 1,326 \\
\hline$-1,330$ & $-1,172$ & 2,342 & 0.0455 & 1.876 & 453 & 918 & 1,111 & 1,286 \\
\hline
\end{tabular}


Table B-14. XCT data for particles in compact LEU09-M36E.

\begin{tabular}{|c|c|c|c|c|c|c|c|c|}
\hline \multicolumn{3}{|c|}{ Centroid $(\boldsymbol{\mu m})$} & \multicolumn{2}{c|}{ Volume $\left(\mathbf{m m}^{\mathbf{3}}\right)$} & \multirow{2}{*}{ Surface distance $(\boldsymbol{\mu m})$} & \multicolumn{3}{c|}{ Nearest neighbor $(\boldsymbol{\mu m})$} \\
\hline $\boldsymbol{y n n n n n n n n n}$\cline { 8 - 9 } & $\boldsymbol{y}$ & $\boldsymbol{z}$ & Kernel & Region & & 1st & 2nd & 3rd \\
\hline 1,431 & 201 & 784 & 0.0440 & 1.948 & 419 & 942 & 1,101 & 1,173 \\
\hline-909 & 884 & 813 & 0.0416 & 2.138 & 453 & 951 & 1,086 & 1,206 \\
\hline 121 & 1,226 & 842 & 0.0393 & 2.015 & 476 & 977 & 1,086 & 1,176 \\
\hline$-1,649$ & -601 & 882 & 0.0405 & 2.375 & 521 & 1,127 & 1,170 & 1,314 \\
\hline 675 & $-1,642$ & 873 & 0.0405 & 2.088 & 510 & 914 & 962 & 1,023 \\
\hline 1,372 & -894 & 886 & 0.0439 & 2.091 & 521 & 1,023 & 1,024 & 1,101 \\
\hline-826 & $-1,830$ & 943 & 0.0430 & 2.261 & 578 & 1,068 & 1,072 & 1,313 \\
\hline 874 & 780 & 1,276 & 0.0474 & 2.015 & 861 & 942 & 977 & 1,052 \\
\hline$-1,414$ & 358 & 1,424 & 0.0428 & 1.816 & 711 & 951 & 995 & 1,089 \\
\hline 71 & $-2,136$ & 1,435 & 0.0453 & 1.566 & 544 & 962 & 1,001 & 1,039 \\
\hline 1,711 & -429 & 1,733 & 0.0448 & 1.761 & 600 & 1,007 & 1,024 & 1,040 \\
\hline$-1,248$ & $-1,269$ & 1,754 & 0.0480 & 1.946 & 768 & 1,031 & 1,059 & 1,072 \\
\hline 951 & $-1,765$ & 1,736 & 0.0433 & 1.839 & 616 & 884 & 914 & 1,001 \\
\hline 262 & 1,260 & 2,009 & 0.0477 & 2.392 & 646 & 1,069 & 1,176 & 1,212 \\
\hline-919 & 988 & 2,015 & 0.0464 & 2.418 & 660 & 995 & 1,206 & 1,212 \\
\hline-138 & -483 & 2,094 & 0.0448 & 3.335 & 612 & 1,402 & 1,474 & 1,516 \\
\hline 1,345 & 415 & 2,143 & 0.0436 & 2.294 & 566 & 1,007 & 1,052 & 1,379 \\
\hline$-1,607$ & -402 & 2,179 & 0.0451 & 2.114 & 532 & 1,031 & 1,089 & 1,314 \\
\hline-569 & $-1,930$ & 2,227 & 0.0449 & 2.033 & 476 & 1,039 & 1,059 & 1,313 \\
\hline 1,345 & $-1,217$ & 2,305 & 0.0426 & 1.782 & 396 & 884 & 1,040 & 1,456 \\
\hline
\end{tabular}


Table B-15. XCT data for particles in compact LEU09-M42E.

\begin{tabular}{|c|c|c|c|c|c|c|c|c|}
\hline \multicolumn{3}{|c|}{ Centroid $(\boldsymbol{\mu m})$} & \multicolumn{2}{c|}{ Volume $\left(\mathbf{m m}^{\mathbf{3}}\right)$} & \multirow{2}{*}{ Surface distance $(\boldsymbol{\mu m})$} & \multicolumn{3}{c|}{ Nearest neighbor $(\boldsymbol{\mu m})$} \\
\hline $\boldsymbol{y n n n n n n n n n}$\cline { 8 - 9 } & $\boldsymbol{y}$ & $\boldsymbol{z}$ & Kernel & Region & & 1st & 2nd & 3rd \\
\hline 129 & 1,128 & 775 & 0.0374 & 1.934 & 430 & 965 & 1,074 & 1,334 \\
\hline$-1,573$ & -581 & 899 & 0.0413 & 2.135 & 555 & 1,040 & 1,076 & 1,305 \\
\hline$-1,101$ & 635 & 931 & 0.0485 & 2.389 & 589 & 1,073 & 1,234 & 1,305 \\
\hline 1,108 & 705 & 903 & 0.0388 & 2.210 & 566 & 1,074 & 1,152 & 1,240 \\
\hline 35 & -401 & 978 & 0.0446 & 2.495 & 634 & 1,121 & 1,478 & 1,537 \\
\hline 1,608 & -426 & 991 & 0.0400 & 2.575 & 657 & 1,240 & 1253 & 1,281 \\
\hline-253 & $-1,914$ & 1,055 & 0.0418 & 2.247 & 713 & 1,089 & 1,094 & 1,094 \\
\hline 810 & $-1,655$ & 1,093 & 0.0469 & 2.524 & 747 & 1,032 & 1,094 & 1,338 \\
\hline$-1,264$ & $-1,548$ & 1,257 & 0.0432 & 2.044 & 563 & 1,076 & 1,094 & 1,142 \\
\hline-419 & 1,165 & 1,569 & 0.0408 & 1.840 & 734 & 904 & 965 & 1,073 \\
\hline$-1,582$ & -229 & 1,878 & 0.0474 & 1.997 & 719 & 938 & 1,028 & 1,040 \\
\hline 1,405 & $-1,334$ & 1,872 & 0.0422 & 1.935 & 591 & 963 & 1,032 & 1,209 \\
\hline 1,413 & 684 & 2,013 & 0.0423 & 2.339 & 556 & 1,152 & 1,207 & 1,253 \\
\hline 108 & -306 & 2,092 & 0.0421 & 2.875 & 702 & 1,121 & 1,553 & 1,573 \\
\hline 302 & 1,255 & 2,107 & 0.0441 & 2.493 & 671 & 904 & 1,253 & 1,349 \\
\hline-405 & $-1,809$ & 2,128 & 0.0459 & 2.047 & 668 & 908 & 1,089 & 1,209 \\
\hline$-1,351$ & 641 & 2,139 & 0.0421 & 2.363 & 635 & 938 & 1,211 & 1,234 \\
\hline 1,644 & -478 & 2,243 & 0.0457 & 1.981 & 555 & 963 & 1,207 & 1,253 \\
\hline$-1,395$ & $-1,141$ & 2,316 & 0.0445 & 1.983 & 487 & 1,028 & 1,142 & 1,209 \\
\hline 467 & $-1,922$ & 2,358 & 0.0461 & 1.873 & 442 & 908 & 1,209 & 1,338 \\
\hline
\end{tabular}


Table B-16. XCT data for particles in compact LEU11-M01B.

\begin{tabular}{|c|c|c|c|c|c|c|c|c|}
\hline \multicolumn{3}{|c|}{ Centroid $(\boldsymbol{\mu m})$} & \multicolumn{2}{c|}{ Volume $\left(\mathbf{m m}^{\mathbf{3}}\right)$} & \multirow{3}{*}{ Surface distance $(\boldsymbol{\mu m})$} & \multicolumn{3}{c|}{ Nearest neighbor $(\boldsymbol{\mu m})$} \\
\hline $\boldsymbol{y n n n n n n n n n}$\cline { 8 - 9 } & $\boldsymbol{y}$ & $\boldsymbol{z}$ & Kernel & Region & & 1st & 2nd & 3rd \\
\hline$-1,156$ & -13 & 827 & 0.0683 & 1.902 & 476 & 946 & 1,046 & 1,330 \\
\hline-295 & $-1,939$ & 852 & 0.0727 & 2.050 & 498 & 944 & 1,231 & 1,373 \\
\hline 1,066 & $-1,678$ & 913 & 0.0757 & 1.880 & 566 & 972 & 1,057 & 1,387 \\
\hline 131 & 1,306 & 930 & 0.0719 & 2.025 & 578 & 1,001 & 1,142 & 1,296 \\
\hline 1,600 & 58 & 937 & 0.0669 & 1.970 & 589 & 995 & 1,040 & 1,231 \\
\hline 44 & -552 & 1,025 & 0.0742 & 2.487 & 668 & 1,176 & 1,330 & 1,438 \\
\hline-951 & 978 & 1,091 & 0.0718 & 2.132 & 647 & 1,046 & 1,142 & 1,227 \\
\hline 925 & 786 & 1,247 & 0.0754 & 2.119 & 815 & 1,001 & 1,021 & 1,040 \\
\hline$-1,738$ & -569 & 1,325 & 0.0827 & 2.164 & 549 & 946 & 1,013 & 1,252 \\
\hline 1,598 & -860 & 1,321 & 0.0778 & 1.826 & 624 & 995 & 1,055 & 1,057 \\
\hline-925 & $-1,693$ & 1,511 & 0.0758 & 2.059 & 675 & 944 & 1,003 & 1,068 \\
\hline 537 & $-1,706$ & 1,728 & 0.0785 & 1.840 & 852 & 955 & 972 & 1,016 \\
\hline-167 & 107 & 1,976 & 0.0772 & 2.018 & 725 & 437 & 1,111 & 1,176 \\
\hline$-1,461$ & $-1,116$ & 2,131 & 0.0769 & 2.206 & 578 & 1,003 & 1,013 & 1,469 \\
\hline$-1,451$ & 353 & 2,123 & 0.0702 & 2.222 & 589 & 1,000 & 1,252 & 1,306 \\
\hline-320 & 1,195 & 2,139 & 0.0774 & 1.949 & 566 & 787 & 1,111 & 1,242 \\
\hline 1,540 & 3 & 2,165 & 0.0754 & 2.190 & 544 & 1,126 & 1,209 & 1,231 \\
\hline 1,335 & $-1,325$ & 2,230 & 0.0760 & 2.049 & 476 & 1,016 & 1,055 & 1,346 \\
\hline-208 & $-2,048$ & 2,218 & 0.0596 & 1.983 & 487 & 955 & 1,068 & 1,373 \\
\hline 949 & 959 & 2,252 & 0.0771 & 1.899 & 453 & 1,021 & 1,126 & 1,296 \\
\hline
\end{tabular}


Table B-17. XCT data for particles in compact LEU11-M05B.

\begin{tabular}{|c|c|c|c|c|c|c|c|c|}
\hline \multicolumn{3}{|c|}{ Centroid $(\boldsymbol{\mu m})$} & \multicolumn{2}{c|}{ Volume $\left(\mathbf{m m}^{\mathbf{3}}\right)$} & \multirow{2}{*}{ Surface distance $(\boldsymbol{\mu m})$} & \multicolumn{3}{c|}{ Nearest neighbor $(\boldsymbol{\mu m})$} \\
\hline $\boldsymbol{y n n n n n n n n n}$\cline { 8 - 9 } & $\boldsymbol{y}$ & $\boldsymbol{z}$ & Kernel & Region & & 1st & 2nd & 3rd \\
\hline$-1,046$ & $-1,573$ & 828 & 0.0753 & 2.065 & 487 & 1,083 & 1,114 & 1,221 \\
\hline 632 & $-1,782$ & 828 & 0.0748 & 1.998 & 487 & 1,042 & 1,094 & 1,278 \\
\hline$-1,310$ & 133 & 872 & 0.0729 & 2.222 & 532 & 1,009 & 1,058 & 1,305 \\
\hline 71 & -326 & 893 & 0.0782 & 2.422 & 555 & 1,205 & 1,410 & 1,456 \\
\hline 1,411 & -764 & 879 & 0.0662 & 2.130 & 544 & 1,043 & 1,093 & 1,283 \\
\hline 1,152 & 838 & 894 & 0.0694 & 2.246 & 555 & 1,115 & 1,123 & 1,315 \\
\hline-352 & 1,196 & 940 & 0.0650 & 2.408 & 600 & 1,101 & 1,230 & 1,243 \\
\hline$-1,589$ & -731 & 1,313 & 0.0727 & 1.952 & 665 & 998 & 1,009 & 1,114 \\
\hline-219 & $-1,972$ & 1,401 & 0.0707 & 1.875 & 671 & 977 & 1,042 & 1,083 \\
\hline 1,680 & 80 & 1,519 & 0.0771 & 1.761 & 571 & 1,057 & 1,072 & 1,093 \\
\hline 1,358 & $-1,513$ & 1,602 & 0.0718 & 1.567 & 512 & 946 & 1,043 & 1,072 \\
\hline 469 & 1,281 & 1,669 & 0.0760 & 1.801 & 607 & 1,020 & 1,066 & 1,101 \\
\hline$-1,196$ & 741 & 1,731 & 0.0661 & 2.056 & 689 & 1,012 & 1,040 & 1,058 \\
\hline$-1,130$ & $-1,541$ & 2,046 & 0.0707 & 2.516 & 623 & 1,185 & 1,197 & 1,221 \\
\hline 147 & -270 & 2,095 & 0.0760 & 2.633 & 578 & 1,205 & 1,470 & 1,552 \\
\hline 450 & $-1,802$ & 2,093 & 0.0709 & 2.258 & 578 & 977 & 1,072 & 1,278 \\
\hline$-1,600$ & -137 & 2,115 & 0.0791 & 2.163 & 555 & 998 & 1,040 & 1,305 \\
\hline 1,610 & -784 & 2,149 & 0.0808 & 1.882 & 521 & 946 & 1,072 & 1,286 \\
\hline-418 & 1,218 & 2,168 & 0.0802 & 1.884 & 510 & 1,012 & 1,020 & 1,230 \\
\hline 1,213 & 736 & 2,204 & 0.0760 & 1.932 & 464 & 1,057 & 1,066 & 1,315 \\
\hline
\end{tabular}


Table B-18. XCT data for particles in compact LEU11-M14B.

\begin{tabular}{|c|c|c|c|c|c|c|c|c|}
\hline \multicolumn{3}{|c|}{ Centroid $(\boldsymbol{\mu m})$} & \multicolumn{2}{c|}{ Volume $\left(\mathbf{m m}^{\mathbf{3}}\right)$} & \multirow{3}{*}{ Surface distance $(\boldsymbol{\mu m})$} & \multicolumn{3}{c|}{ Nearest neighbor $(\boldsymbol{\mu m})$} \\
\hline $\boldsymbol{y n n n n n n n n n}$\cline { 8 - 9 } & $\boldsymbol{y}$ & $\boldsymbol{z}$ & Kernel & Region & & 1st & 2nd & 3rd \\
\hline$-1,432$ & $-1,122$ & 826 & 0.0649 & 2.068 & 521 & 1,116 & 1,116 & 1,300 \\
\hline 847 & $-1,513$ & 824 & 0.0665 & 1.809 & 521 & 963 & 981 & 1,206 \\
\hline$-1,101$ & 667 & 842 & 0.0683 & 2.239 & 532 & 1,067 & 1,093 & 1,288 \\
\hline 338 & 1,258 & 859 & 0.0632 & 2.236 & 555 & 1,062 & 1,176 & 1,261 \\
\hline 1,543 & 67 & 886 & 0.0764 & 2.150 & 578 & 1,067 & 1,121 & 1,257 \\
\hline 25 & -303 & 932 & 0.0694 & 2.519 & 623 & 1,149 & 1,466 & 1,489 \\
\hline-320 & $-1,780$ & 970 & 0.0807 & 2.329 & 668 & 1,180 & 1,197 & 1,206 \\
\hline 1,634 & -989 & 1,008 & 0.0689 & 1.767 & 541 & 963 & 1,067 & 1,112 \\
\hline$-1,664$ & -128 & 1,276 & 0.0761 & 1.997 & 629 & 1,001 & 1,067 & 1,116 \\
\hline 1,157 & 949 & 1,460 & 0.0703 & 1.761 & 593 & 1,028 & 1,061 & 1,062 \\
\hline-567 & 1,231 & 1,611 & 0.0771 & 1.993 & 659 & 1,014 & 1,093 & 1,132 \\
\hline 698 & $-1,910$ & 1,709 & 0.0765 & 1.845 & 567 & 981 & 995 & 1,135 \\
\hline$-1,143$ & $-1,595$ & 1,795 & 0.0685 & 2.013 & 593 & 1,059 & 1,116 & 1,133 \\
\hline 165 & -86 & 2,052 & 0.0712 & 2.471 & 600 & 1,149 & 1,404 & 1,412 \\
\hline 1,343 & $-1,053$ & 2,079 & 0.0751 & 2.285 & 566 & 1,112 & 1,135 & 1,288 \\
\hline$-1,297$ & 738 & 2,113 & 0.0801 & 2.079 & 532 & 1,014 & 1,259 & 1,285 \\
\hline 444 & 1,297 & 2,115 & 0.0691 & 1.976 & 532 & 1,028 & 1,132 & 1,261 \\
\hline 1,533 & 220 & 2,134 & 0.0788 & 2.093 & 521 & 1,061 & 1,257 & 1,288 \\
\hline$-1,386$ & -544 & 2,143 & 0.0749 & 2.197 & 510 & 1,001 & 1,133 & 1,285 \\
\hline-190 & $-1,883$ & 2,156 & 0.0717 & 1.974 & 498 & 995 & 1,059 & 1,197 \\
\hline
\end{tabular}




\section{APPENDIX C.}

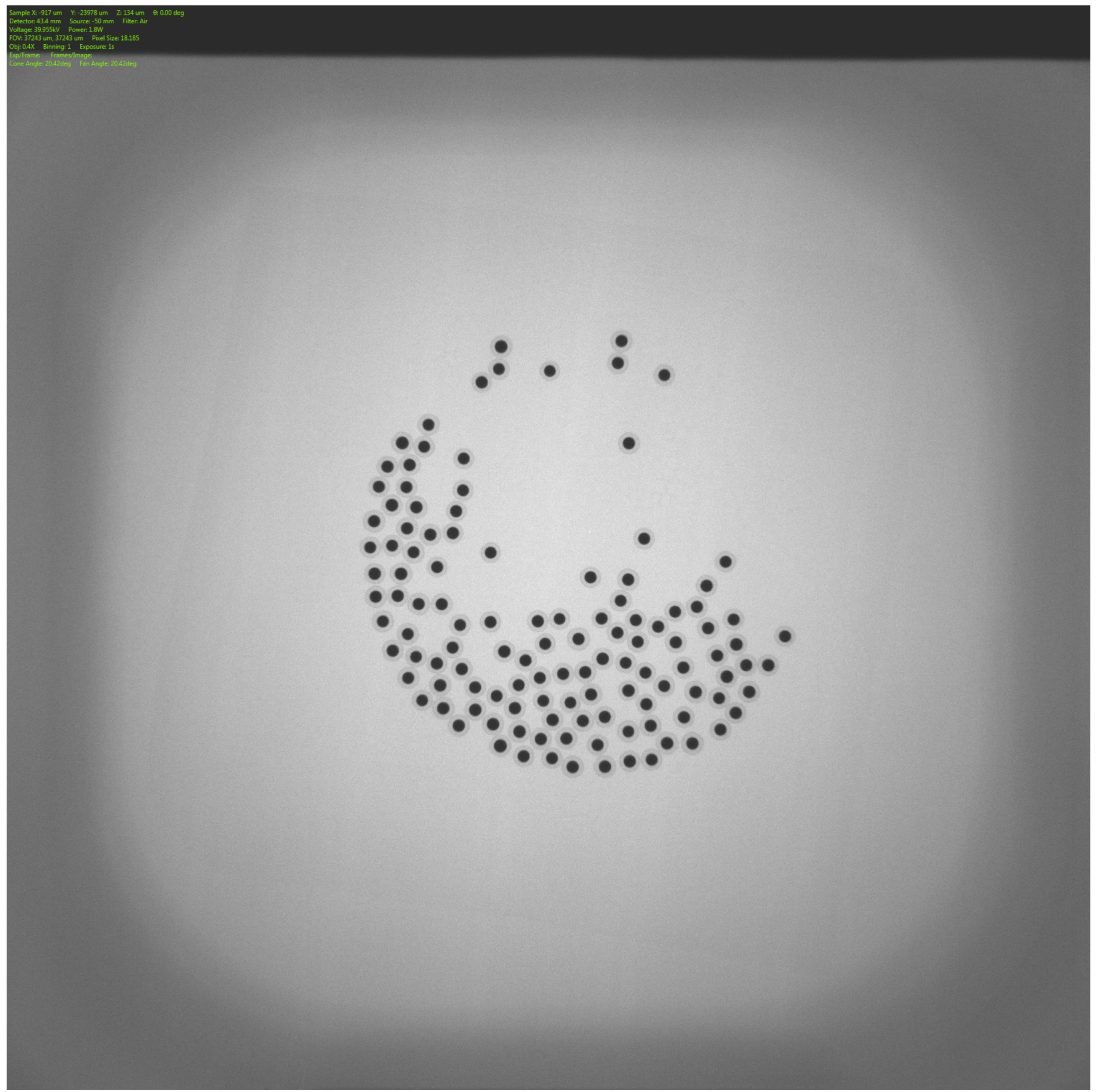

Figure C-1. Radiograph of burn test clutch \#1 from LEU09-M\#\#E. 


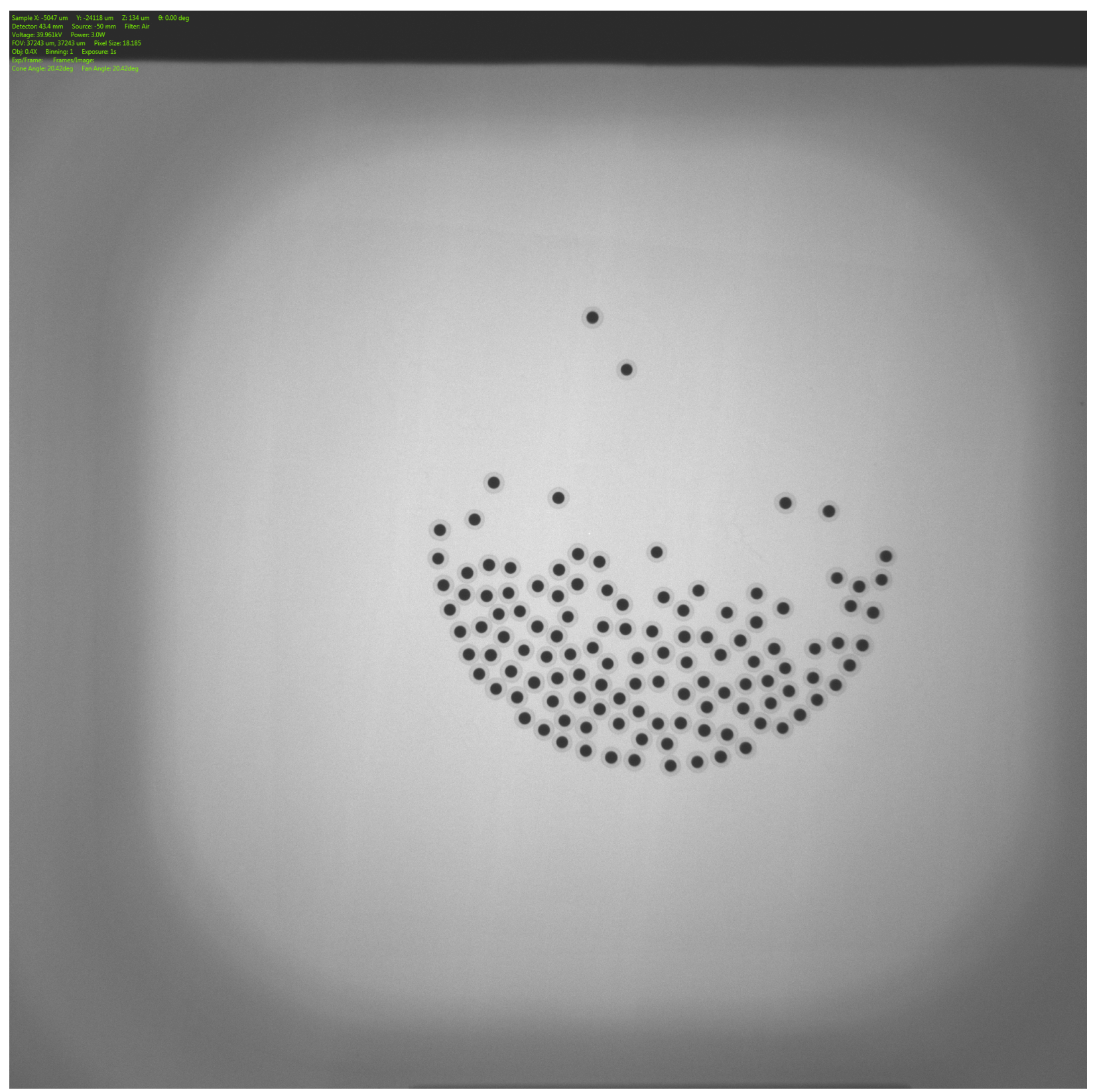

Figure C-2. Radiograph of burn test clutch \#2 from LEU09-M\#\#E. 


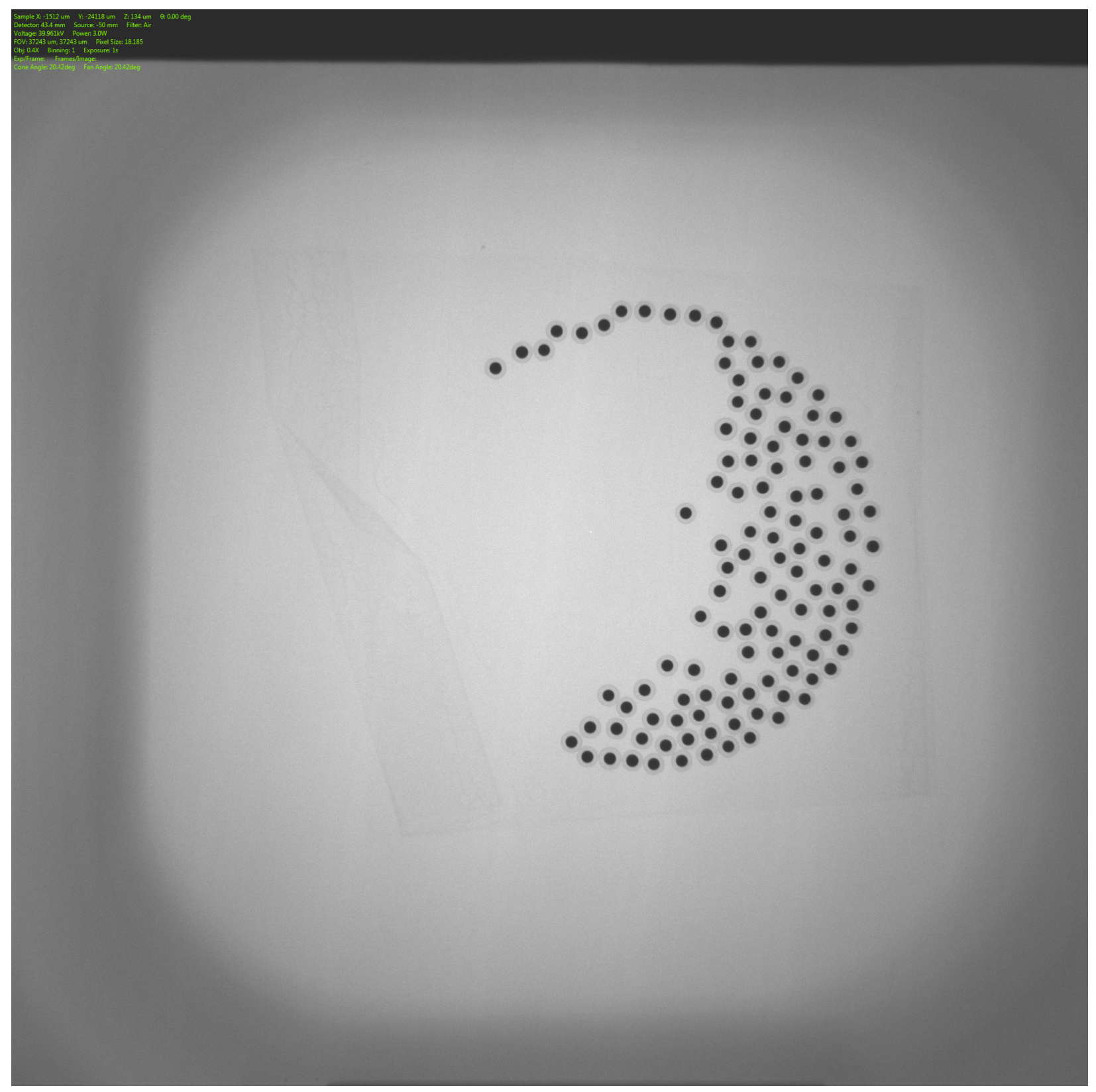

Figure C-3. Radiograph of burn test clutch \#3 from LEU09-M\#\#E. 


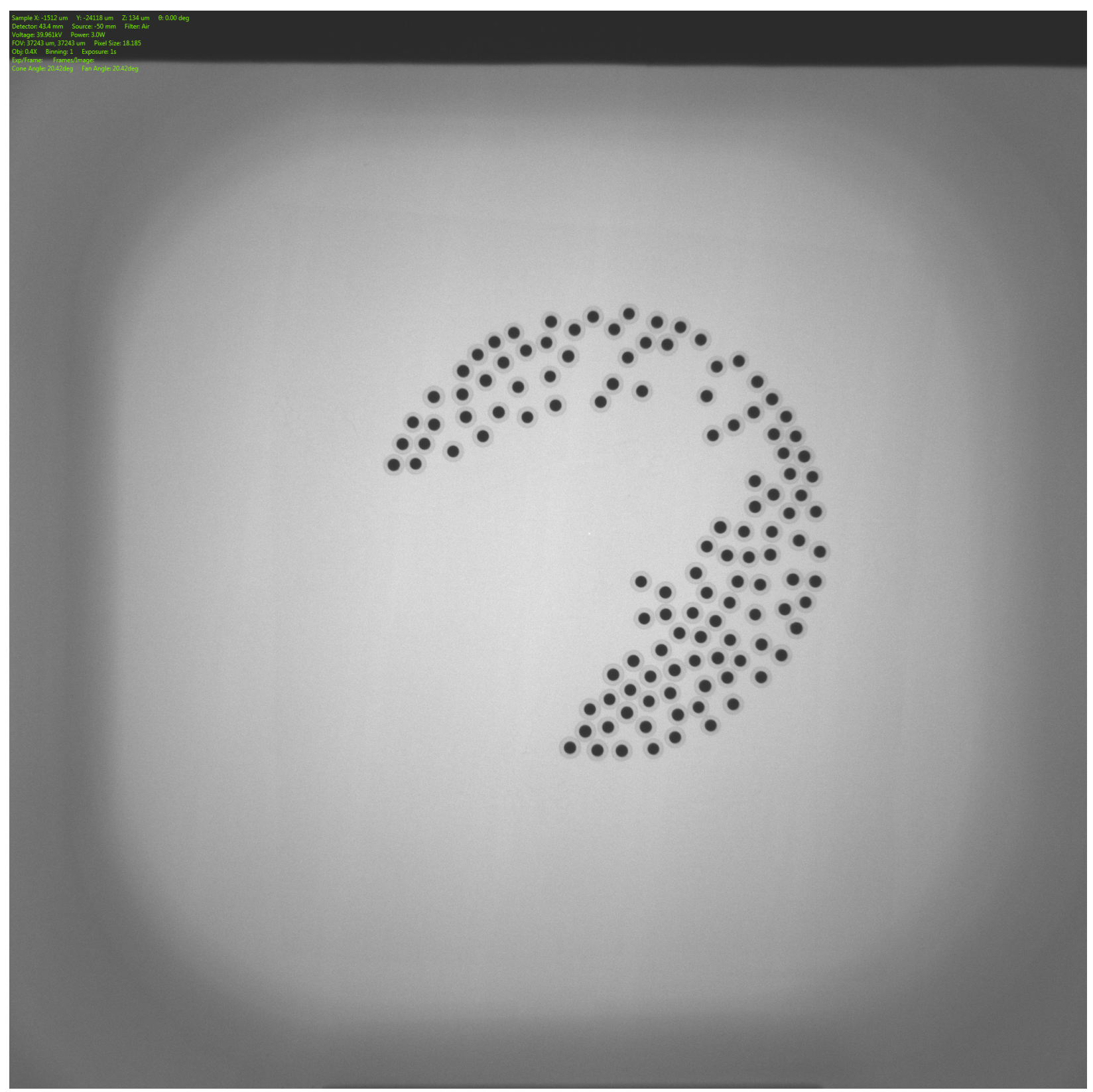

Figure C-4. Radiograph of burn test clutch \#1 from LEU09-M\#\#G. 


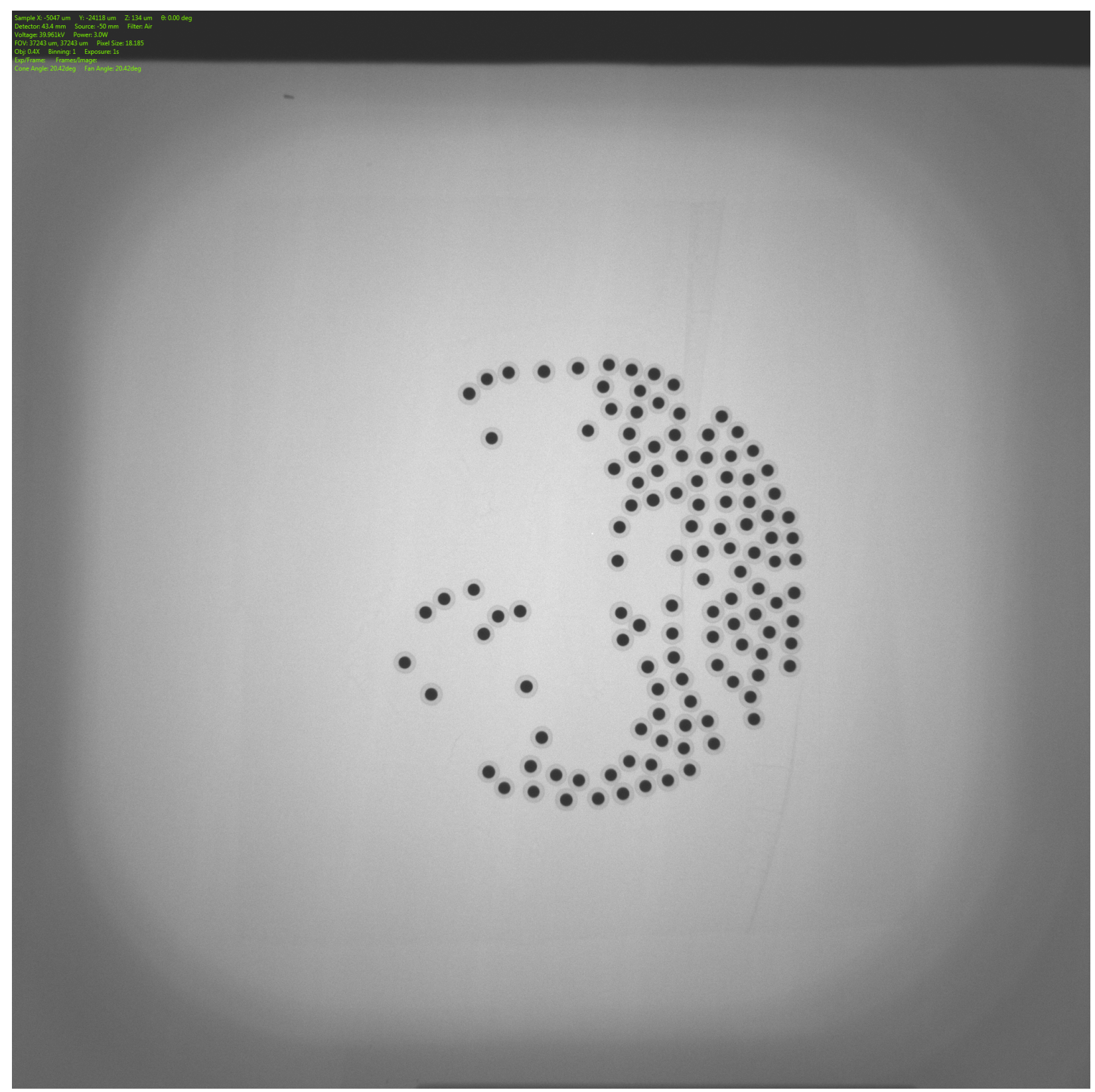

Figure C-5. Radiograph of burn test clutch \#2 from LEU09-M\#\#G. 


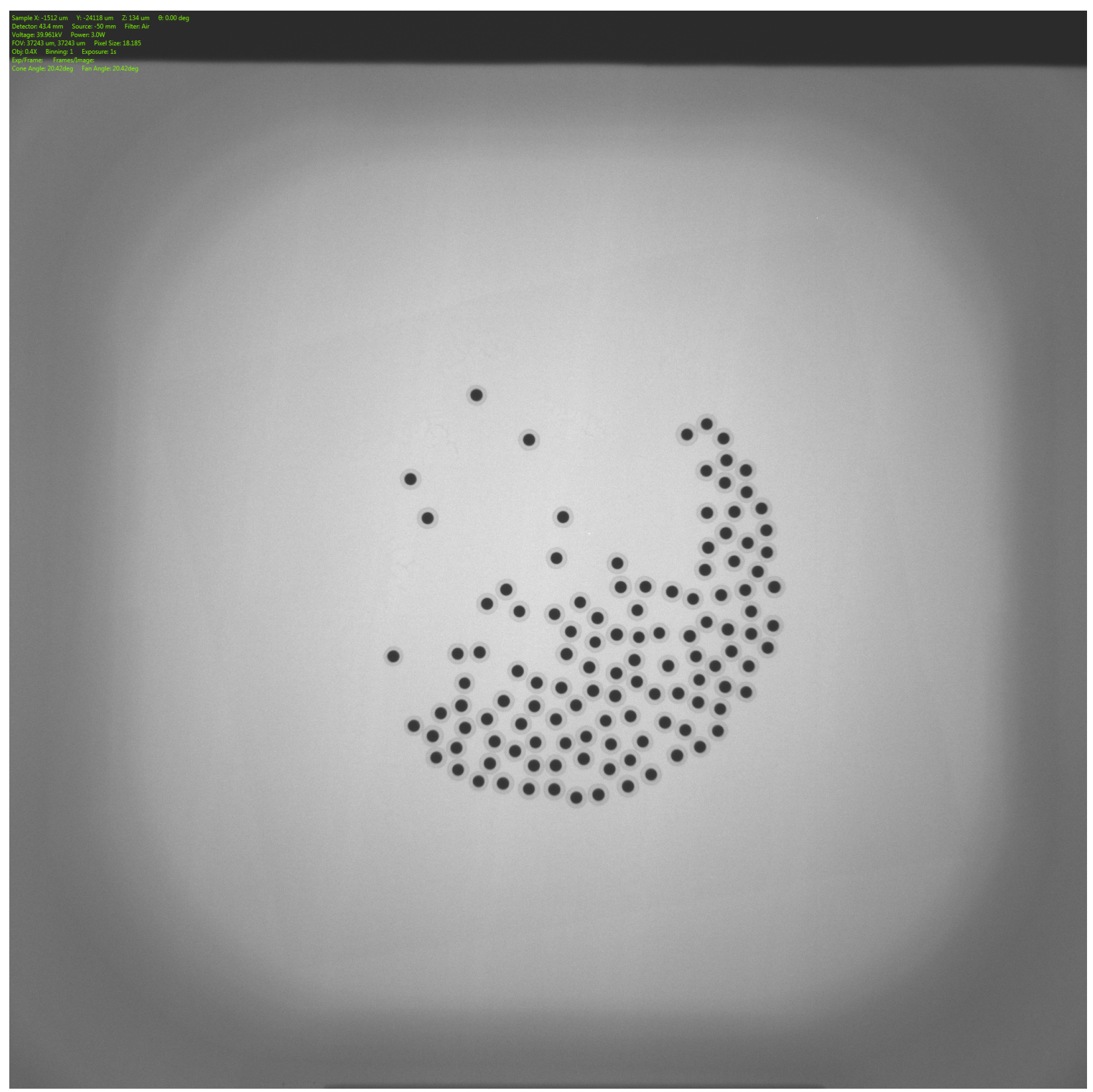

Figure C-6. Radiograph of burn test clutch \#3 from LEU09-M\#\#G. 


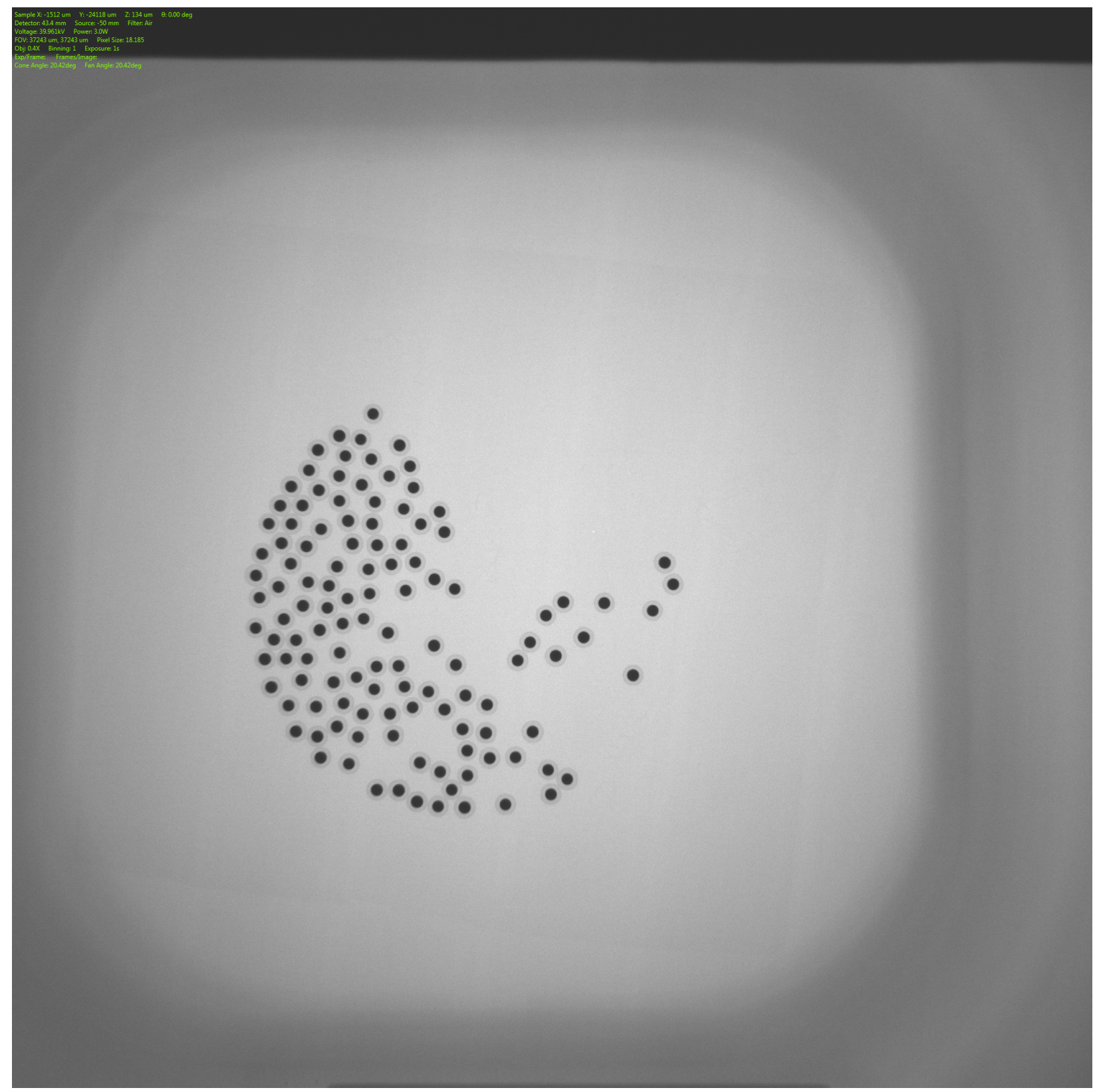

Figure C-7. Radiograph of burn test clutch \#4 from LEU09-M\#\#G. 


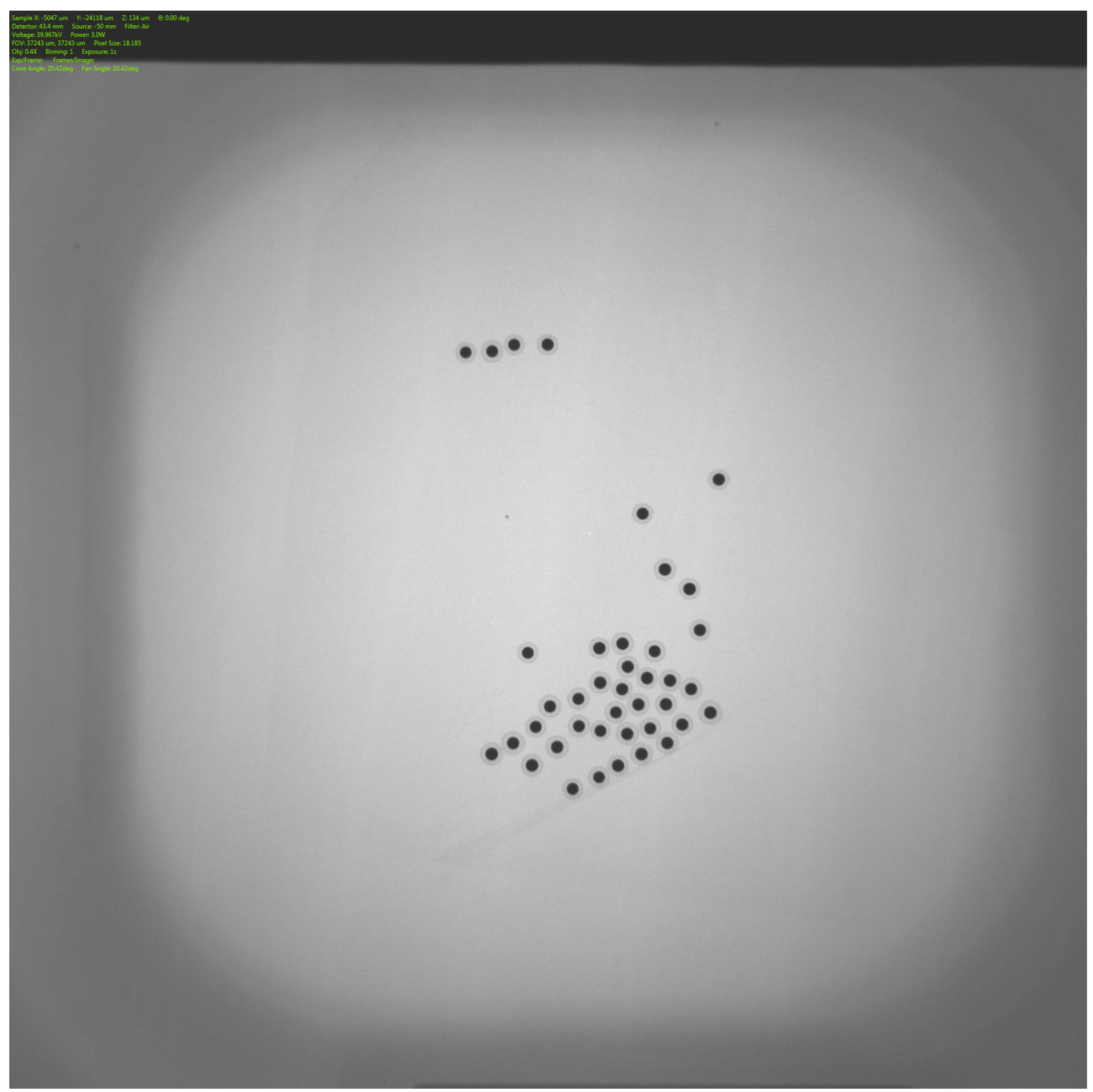

Figure C-8. Radiograph of burn test clutch \#5 from LEU09-M\#\#G. 


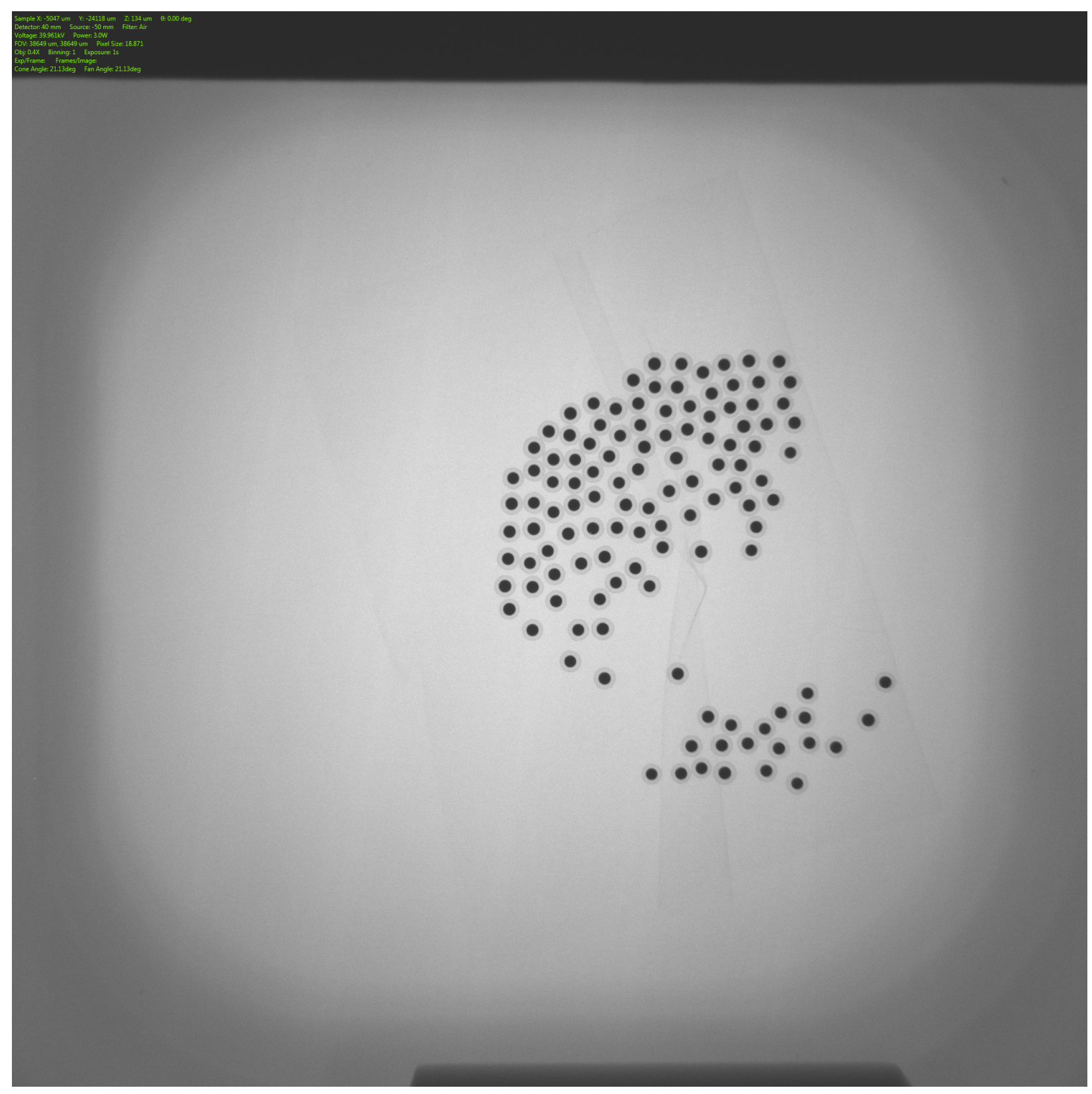

Figure C-9. Radiograph of burn test clutch \#6 from LEU09-M\#\#G. 


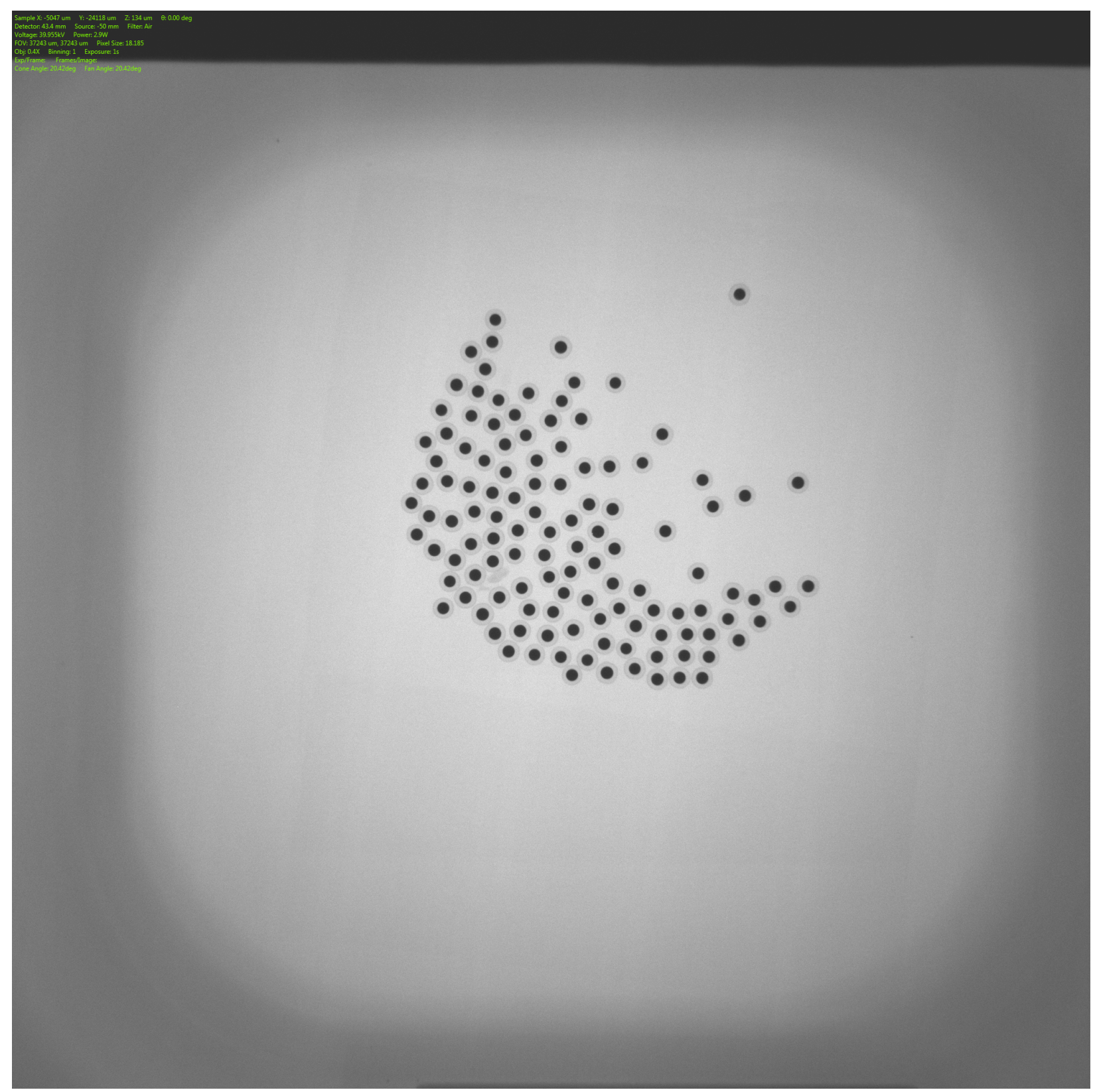

Figure C-10. Radiograph of burn test clutch \#7 from LEU09-M\#\#. 


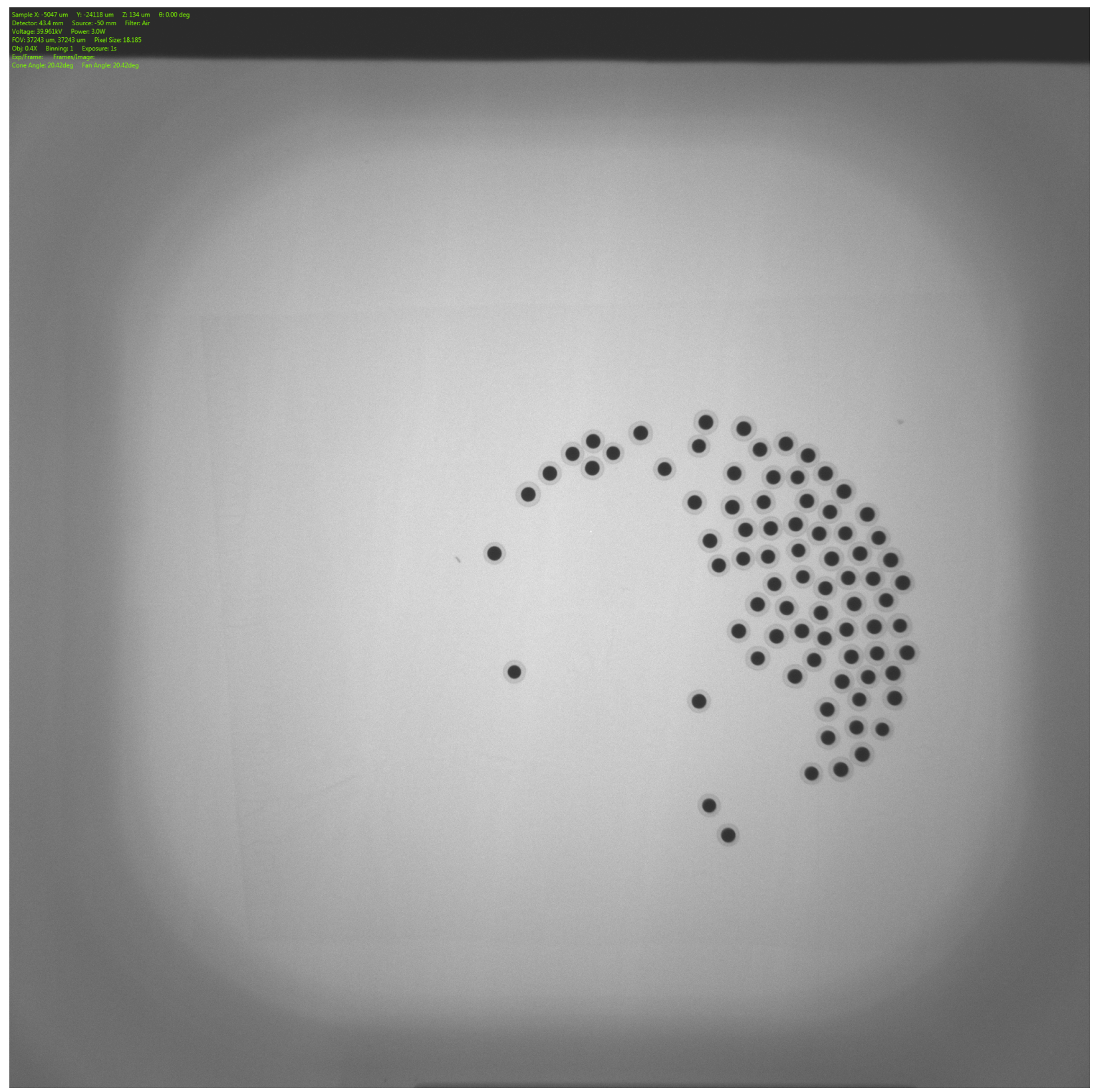

Figure C-11. Radiograph of burn test clutch \#1 from LEU11-M\#\#B. 


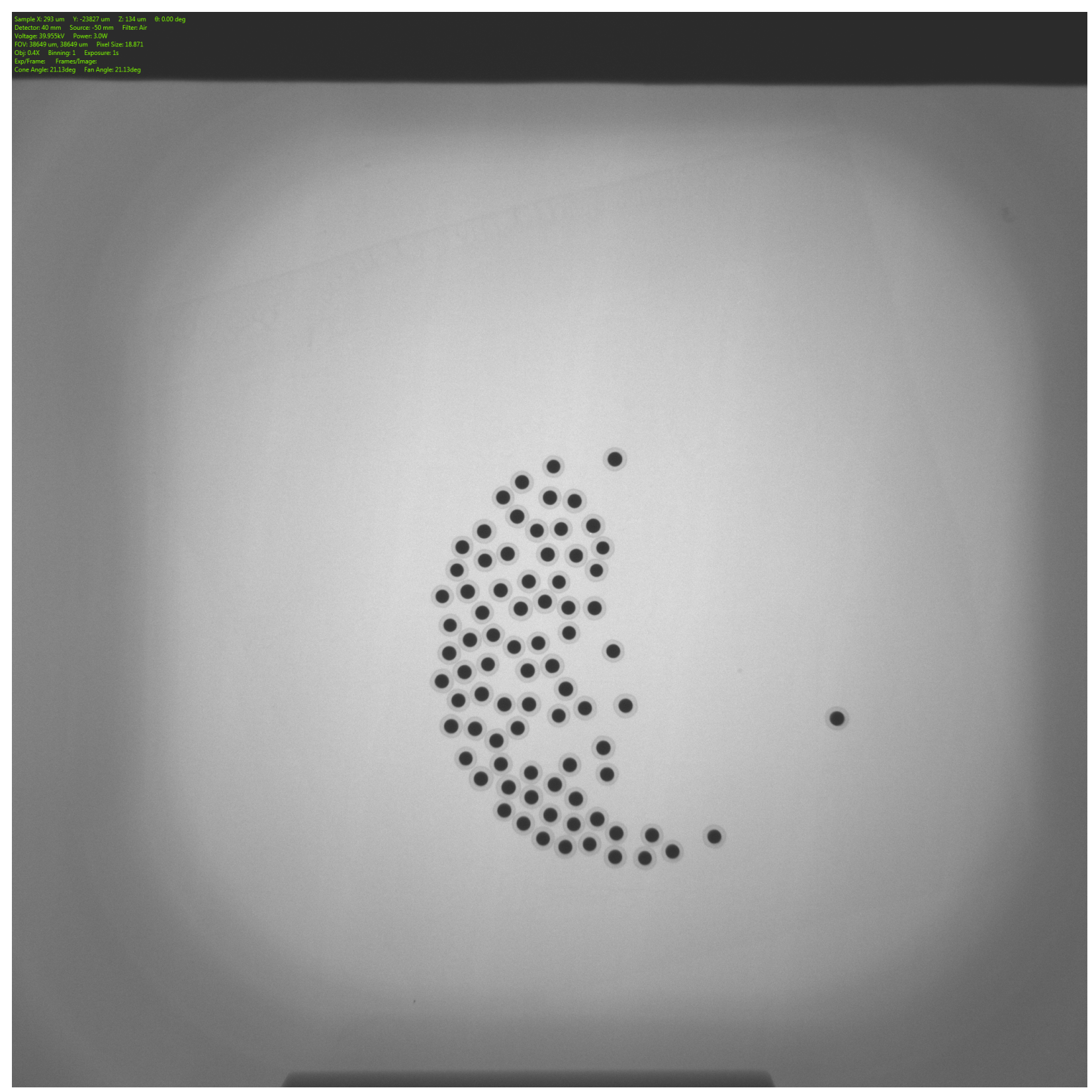

Figure C-12. Radiograph of burn test clutch \#2 from LEU11-M\#\#B. 


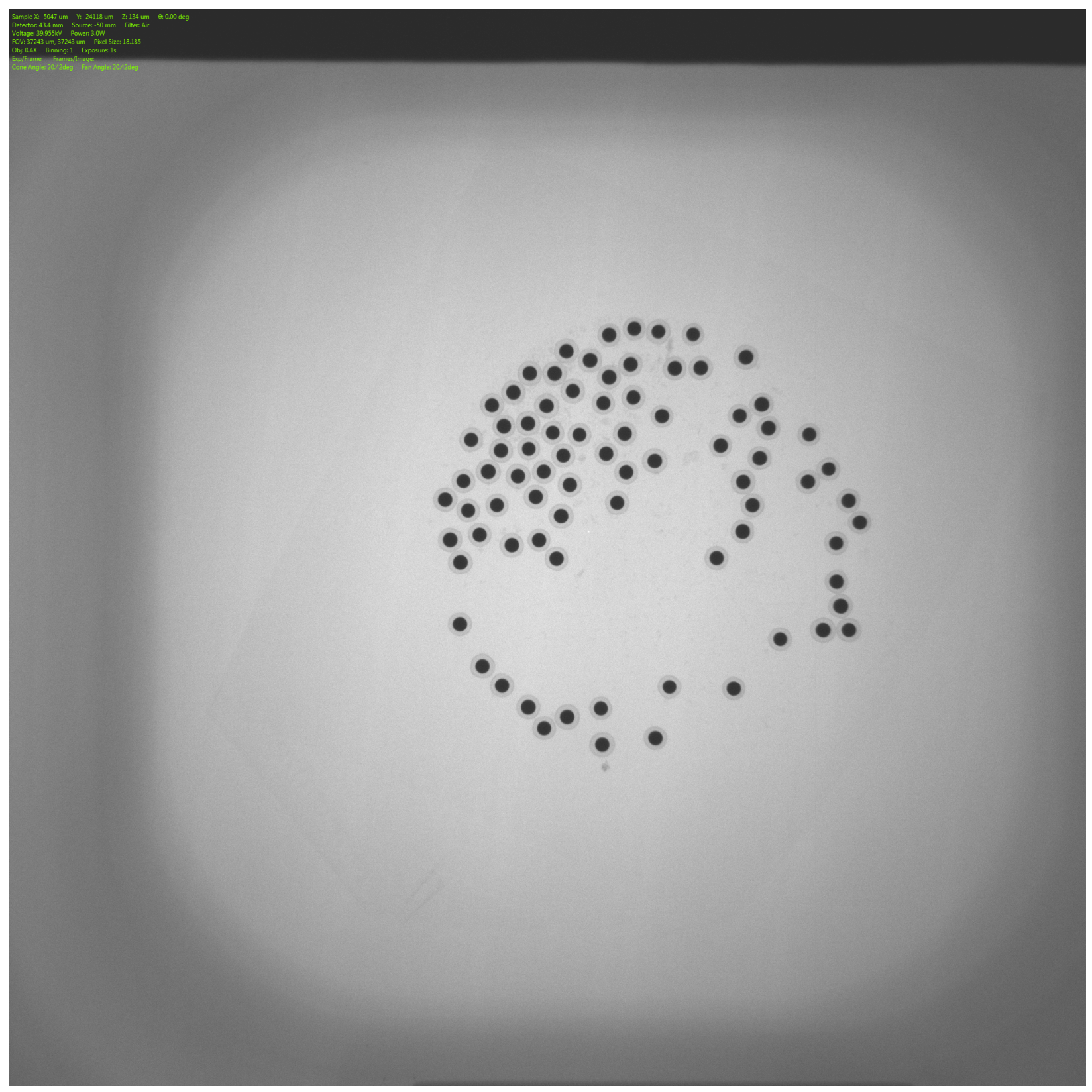

Figure C-13. Radiograph of burn test clutch \#3 from LEU11-M\#\#B. 
